\title{
(CO)CYCLIC (CO)HOMOLOGY OF BIALGEBROIDS: AN APPROACH VIA (CO)MONADS
}

\author{
GABRIELLA BÖHM AND DRAGOŞ ŞTEFAN
}

\begin{abstract}
For a (co)monad $\boldsymbol{T}_{l}$ on a category $\mathcal{M}$, an object $X$ in $\mathcal{M}$, and a functor $\boldsymbol{\Pi}: \mathcal{M} \rightarrow \mathcal{C}$, there is a (co)simplex $Z^{*}:=\boldsymbol{\Pi} \boldsymbol{T}_{l}{ }^{*+1} X$ in $\mathcal{C}$. The aim of this paper is to find criteria for para(co)cyclicity of $Z^{*}$. Our construction is built on a distributive law of $\boldsymbol{T}_{l}$ with a second (co)monad $\boldsymbol{T}_{\boldsymbol{r}}$ on $\mathcal{M}$, a natural transformation $\boldsymbol{i}: \boldsymbol{\Pi} \boldsymbol{T}_{\boldsymbol{l}} \rightarrow \boldsymbol{\Pi} \boldsymbol{T}_{\boldsymbol{r}}$, and a morphism $w: \boldsymbol{T}_{\boldsymbol{r}} X \rightarrow \boldsymbol{T}_{\boldsymbol{l}} X$ in $\mathcal{M}$. The (symmetrical) relations $\boldsymbol{i}$ and $w$ need to satisfy are categorical versions of Kaygun's axioms of a transposition map. Motivation comes from the observation that a (co)ring $T$ over an algebra $R$ determines a distributive law of two (co)monads $\boldsymbol{T}_{\boldsymbol{l}}=T \otimes_{R}(-)$ and $\boldsymbol{T}_{\boldsymbol{r}}=(-) \otimes_{R} T$ on the category of $R$-bimodules. The functor $\Pi$ can be chosen such that $Z^{n}=T \widehat{\otimes}_{R} \ldots \widehat{\otimes}_{R} T \widehat{\otimes}_{R} X$ is the cyclic $R$-module tensor product. A natural transformation $i: T \widehat{\otimes}_{R}(-) \rightarrow(-) \widehat{\otimes}_{R} T$ is given by the flip map and a morphism $w: X \otimes_{R} T \rightarrow T \otimes_{R} X$ is constructed whenever $T$ is a (co)module algebra or coring of an $R$-bialgebroid. The notion of a stable anti Yetter-Drinfel'd module over certain bialgebroids, so called $\times_{R}$-Hopf algebras, is introduced. In the particular example when $T$ is a module coring of a $\times_{R}$-Hopf algebra $\mathcal{B}$ and $X$ is a stable anti Yetter-Drinfel'd $\mathcal{B}$-module,

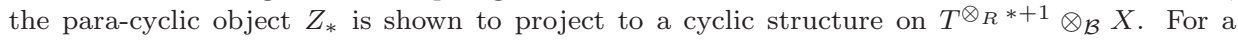
$\mathcal{B}$-Galois extension $S \subseteq T$, a stable anti Yetter-Drinfel'd $\mathcal{B}$-module $T_{S}$ is constructed, such that the cyclic objects $\mathcal{B}^{\otimes_{R}{ }^{*+1}} \otimes_{\mathcal{B}} T_{S}$ and $T^{\widehat{\otimes}_{S}{ }^{*+1}}$ are isomorphic. This extends a theorem by Jara and Ştefan for Hopf Galois extensions. As an application, we compute Hochschild and cyclic homologies of a groupoid with coefficients in a stable anti Yetter-Drinfel'd module, by tracing it back to the group case. In particular, we obtain explicit expressions for (coinciding relative and ordinary) Hochschild and cyclic homologies of a groupoid. Latter extends results of Burghelea on cyclic homology of groups.
\end{abstract}

\section{Contents}

Introduction

1. The (co)cyclic object associated to a transposition map 4

1.A. Notation and conventions 4

1.B. Monads and distributive laws 4

1.C. Admissible septuples and transposition maps. The main result. 6

1.D. Examples of admissible septuples and transposition maps. Applications. 9

1.E. The dual construction 12

2. Cyclic (co)homology of bialgebroids 15

2.A. (Co)module algebras of bialgebroids 16

2.B. (Co)module corings of bialgebroids 19

2.C. Stable anti Yetter-Drinfel'd modules of $\times_{R}$-Hopf algebras 20

2.D. Galois extensions of $\times_{R}$-Hopf algebras 24

3. Cyclic homology of groupoids 28

3.A. Anti Yetter-Drinfel'd modules for groupoids 28

3.B. Hochschild and cyclic homology with coefficients 31

3.C. Cyclic homology of groupoids 33

Acknowledgments $\quad 37$

References $\quad 38$

Date: July 2007.

2000 Mathematics Subject Classification. Primary 16W30; Secondary 16E40.

Key words and phrases. (co)monads, para-(co)cyclic objects, bialgebroids, $\times_{R}$-Hopf algebras, Galois extensions, Hochschild and cyclic homologies of groupoids. 


\section{INTRODUCTION}

Cyclic cohomology of Hopf algebras is originated from the work [CM98] of Connes and Moscovici on the index theory of transversally elliptic operators. Their local index formula in [CM95] gives a generalization of the Chern character to non-commutative geometry. In order to give a geometrical interpretation of the non-commutative Chern character in terms of non-commutative foliations, in [CM98] a cocyclic structure was constructed on a cosimplex $Z_{n}^{C M}=H^{\otimes n}$, associated to the coalgebra underlying a Hopf algebra $H$ over a field $\mathbb{K}$. The cocyclic operator was given in terms of a so called modular pair in involution.

In the subsequent years the Connes-Moscovici cocyclic module was placed in a broader and broader context. In [KR03] (see also [HKRS2]) to any (co)module algebra $T$ of a Hopf algebra $H$, and any $H$-(co)module $X$, there was associated a para-cyclic module with components $T^{\otimes *+1} \otimes X$. Dually, for any (co)module coalgebra $T$ of a Hopf algebra $H$, and any $H$-(co)module $X$, there is a para-cocyclic module with components $T^{\otimes *+1} \otimes X$. The Connes-Moscovici cosimplex $Z_{*}^{C M}$ turns out to be isomorphic to a quotient of the para-cocyclic module associated to the regular module coalgebra $T:=H$ and an $H$-comodule defined on $\mathbb{K}$. For bialgebras, the Connes-Moscovici construction was generalized in [Kay05].

In the papers [HKRS1] and [JS], a modular pair in involution was proven to be equivalent to a stable anti Yetter-Drinfel'd module structure on the ground field $\mathbb{K}$. In [HKRS2], the para-cocyclic module $T^{\otimes *+1} \otimes X$, associated to an $H$-module coalgebra $T$ and a stable anti Yetter-Drinfel'd $H$-module $X$, was shown to project to a cocyclic object whose components are the $H$-module tensor products $T^{\otimes *+1} \otimes_{H} X$. The way in which the para-cocyclic object $H^{\otimes *+1}$ projects to the Connes-Moscovici cosimplex $Z_{*}^{C M}$, is an example of this scenario. Dually, the para-cyclic module, associated to an $H$-comodule algebra, was proven to have a cyclic submodule.

In the spirit of [JS], one can follow a dual approach. That is, para-cocyclic modules can be constructed for (co)module algebras of Hopf algebras, and para-cyclic modules for (co)module coalgebras, in both cases with coefficients in $H$-(co)modules. Taking coefficients in a stable anti Yetter Drinfel'd module, it was shown in [JŞ] that in this case the para-cyclic object associated to a module coalgebra possesses a cyclic quotient. In [KR05] an isomorphism was proven between the cyclic quotient of the para-cyclic object in [JS] of $H$ as an $H$-module coalgebra on one hand, and the cyclic subobject of the para-cyclic object in [HKRS2] of $H$ as an $H$-comodule algebra on the other.

Constructions in Section 2 of the current paper follow the root in [JS]. Since this framework is dual to that suggested in [HKRS2] (cf. also [Kay06]), some might like to call it a dual Hopf (co)cyclic theory. However, we do not use this somewhat involved terminology in the paper, but remind the reader to the difference between the two possible dual approaches.

In [Kay06] Kaygun proposed a unifying approach to the para-(co)cyclic objects corresponding to a (co)module (co)algebra of a Hopf algebra. Starting with a (co)algebra $T$ and an object $X$ in a symmetric monoidal category $\mathcal{S}$, he introduced the notion of a transposition map. It is a morphism $w: X \otimes T \rightarrow T \otimes X$ in $\mathcal{S}$, satisfying conditions reminiscent to half of the axioms of a distributive law in [Be]. Any transposition map $w$ was shown to determine a para-(co)cyclic structure on the (co)simplex $T^{\otimes *+1} \otimes X$ in $\mathcal{S}$. In particular, canonical transposition maps were constructed for (co)module (co)algebras $T$ and (co)modules $X$ of a bialgebra.

Connes and Moscovici's index theory of transversally elliptic operators lead beyond cyclic homology of Hopf algebras. In dealing with the general, non-flat case, in [CM01] certain bialgebroids (in fact $\times_{R}$-Hopf algebras) arose naturally. Bialgebroids can be thought of as a generalization of bialgebras to a non-commutative base algebra $R$, while $\times_{R}$-Hopf algebras generalize Hopf algebras. There are a few papers in the literature, e.g. [KR04] and [Ra], attempting to extend Hopf cyclic theory to non-commutative base algebras. However, an understanding of the subject, comparable to that in the classical case of a commutative base ring (or field), is missing yet. The aim of the current paper is to give a universal construction of para-(co)cyclic (co)simplices, including examples coming from (co)module algebras and (co)module corings for bialgebroids.

When replacing bialgebras over a commutative ring $\mathbb{K}$ by bialgebroids over a non-commutative $\mathbb{K}$-algebra $R$, the monoidal category of $\mathbb{K}$-modules becomes replaced by the monoidal category 
of $R$-bimodules. Indeed, (co)module algebras of an $R$-bialgebroid are in particular algebras, and (co)module corings are coalgebras, in the category of $R$-bimodules. The main difference is that the category of $\mathbb{K}$-modules is symmetric. In contrast, the category of $R$-bimodules is not even braided in general. Hence Kaygun's elegant theory [Kay06], formulated in a symmetric monoidal category $\mathcal{S}$, is not applicable.

Our key observation is that the role, the symmetry plays in Kaygun's work, is that it defines a compatible natural transformation $\boldsymbol{i}$ between the two (co)monads $T \otimes(-)$ and $(-) \otimes T$ on the symmetric monoidal category $\mathcal{S}$, induced by a (co)algebra $T$ in $\mathcal{S}$. Note that these (co)monads on $\mathcal{S}$ are connected by a trivial distributive law. Guided by this observation, in Section 1 we start with a distributive law of two (co)monads $\boldsymbol{T}_{\boldsymbol{l}}$ and $\boldsymbol{T}_{\boldsymbol{r}}$ on any category $\mathcal{M}$. In addition, we allow for the presence of a functor $\Pi: \mathcal{M} \rightarrow \mathcal{C}$ (it is the identity functor on $\mathcal{S}$ in [Kay06]). Then, for any object $X$ in $\mathcal{M}$, there is a (co)simplex in $\mathcal{C}$, given at degree $n$ by $\boldsymbol{\Pi} \boldsymbol{T}_{l}{ }^{n+1} X$. In Sections 1.C and 1.E we show that it is para-(co)cyclic provided that there exist a natural transformation $\boldsymbol{i}: \boldsymbol{\Pi} \boldsymbol{T}_{l} \rightarrow \boldsymbol{\Pi} \boldsymbol{T}_{\boldsymbol{r}}$ and a morphism $\boldsymbol{T}_{\boldsymbol{r}} X \rightarrow \boldsymbol{T}_{l} X$ in $\mathcal{M}$, satisfying symmetrical conditions generalizing Kaygun's axioms of a transposition map. Examples of this situation are collected in Section 1.D. Among other (classical) examples, we show that Škoda's functorial construction in [Šk] of a paracyclic object in the category of endofunctors, Majid and Akrami's para-cyclic modules associated to a ribbon algebra in [AM], and Rangipour's cyclic module in [Ra] determined by a coring, fit our framework. It is discussed in Sections 2.A and 2.B how the general results in Sections 1.C and 1.E cover the particular cases when the two (co)monads $\boldsymbol{T}_{\boldsymbol{l}}=T \otimes_{R}(-)$ and $\boldsymbol{T}_{\boldsymbol{r}}=(-) \otimes_{R} T$ are induced by a (co)module algebra or (co)module coring $T$ of an $R$-bialgebroid $\mathcal{B}$, the functor $\Pi$ is defined via the coequalizer of the $R$-actions in a bimodule, and $X$ is a $\mathcal{B}$-(co)module. The components of the resulting para-(co)cyclic module are cyclic $R$-module tensor products $T \widehat{\otimes}_{R} \ldots \widehat{\otimes}_{R} T \widehat{\otimes}_{R} X$. In this way we obtain examples which extend both some para-(co)cyclic objects in [Kay06] and [HKRS2] and their cyclic duals.

By the above procedure, we associate a para-cyclic object in particular to a module coring $C$ and a comodule $X$ of an $R$-bialgebroid $\mathcal{B}$. Following [JS3], in Section 2.C we look for situations in which it projects to the $\mathcal{B}$-module tensor product $\left(C \otimes_{R} \cdots \otimes_{R} C\right) \otimes_{\mathcal{B}} X$. Restricting at this point our study to $\times_{R}$-Hopf algebras $\mathcal{B}$, we define stable anti Yetter-Drinfel'd modules for $\mathcal{B}$. In parallel to the case of Hopf algebras [JSS, Theorem 4.13], [HKRS2, Theorem 2.1], we prove cyclicity of the simplex $\left(C \otimes_{R} \cdots \otimes_{R} C\right) \otimes_{\mathcal{B}} X$, whenever $X$ is a stable anti Yetter-Drinfel'd module.

A simplest example of a cyclic simplex is associated to an algebra extension $S \subseteq T$. Its components are given by the $n+1$ fold cyclic tensor product $T^{\widehat{\otimes}_{S} n+1}$, face and degeneracy maps are determined by the algebra structure of $T$ and the cyclic map is given by the cyclic permutation of the tensor factors. In Section 2.D, for a Galois extension $S \subseteq T$ by a $\times_{R}$-Hopf algebra $\mathcal{B}$, we construct a stable anti Yetter-Drinfel'd module $T_{S}:=T /\{s \cdot t-t \cdot s \mid s \in S, t \in T\}$. We prove that the cyclic simplices $T^{\widehat{\otimes}_{S} n+1}$ and $\mathcal{B}^{\otimes_{R} n+1} \otimes_{\mathcal{B}} T_{S}$ are isomorphic. This extends [JSS, Theorem 3.7].

A most fundamental class of examples of bialgebroids (in fact $\times_{R}$ Hopf algebras) is given by algebras (over fields), generated by a groupoid of finitely many objects. As an application of our abstract theory, we compute explicitly the relative Hochschild and cyclic homologies of such a groupoid, with coefficients in a stable anti Yetter-Drinfel'd module. By our results, any Galois extension by the groupoid provides us with a stable anti Yetter-Drinfel'd module. In particular, the groupoid algebra $\mathcal{B}$ is a Galois extension of its base algebra $R$. Applying the isomorphism of the simplices $\mathcal{B}^{\widehat{\otimes}_{R} n+1}$ and $\mathcal{B}^{\otimes_{R}}{ }^{n+1} \otimes_{\mathcal{B}} \mathcal{B}_{R}$, we obtain the $R$-relative cyclic homology of $\mathcal{B}$. Since $R$ is a separable algebra, it is equal to ordinary cyclic homology of $\mathcal{B}$, hence our results extend those by Burghelea on the cyclic homology of groups [Burg]. Similar formulae were obtained by Crainic for cyclic homology of étale groupoids [Cra]. Observe that any groupoid (with arbitrary set of objects) can be obtained as a direct limit of groupoids with finite sets of objects. Certainly, the algebra generated by a groupoid with infinitely many objects is no longer unital. However, one can still consider its cyclic homology, as a homology of Connes' complex, associated to a presimplicial object. Since the homology functor commutes with direct limits, we can extend our formula of cyclic homology to arbitrary groupoids. 
Throughout the paper $\mathbb{K}$ denotes a commutative and associative unital ring. The term $\mathbb{K}$-algebra means an associative and unital algebra over $\mathbb{K}$.

\section{The (CO)CYCLIC OBJECT ASSOCIATED TO A TRANSPOSITION MAP}

In this first section we establish a general categorical framework - in terms of admissible septuples and their transposition maps - to produce para-cocyclic, and dually, para-cyclic objects.

1.A. Notation and conventions. In the 2-category CAT we denote horizontal composition (of functors) by juxtaposition, while $\circ$ is used for vertical composition (of natural transformations). That is, for two functors $\boldsymbol{F}: \mathcal{C} \rightarrow \mathcal{C}^{\prime}, \boldsymbol{G}: \mathcal{C}^{\prime} \rightarrow \mathcal{C}^{\prime \prime}$ and an object $X$ in $\mathcal{C}$, instead of $\boldsymbol{G}(\boldsymbol{F}(X))$ we write $\boldsymbol{G} \boldsymbol{F} X$. For two natural transformations $\boldsymbol{\mu}: \boldsymbol{F} \rightarrow \boldsymbol{F}^{\prime}$ and $\boldsymbol{\nu}: \boldsymbol{G} \rightarrow \boldsymbol{G}^{\prime}$ we write $\boldsymbol{G}{ }^{\prime} \boldsymbol{\mu} X \circ \boldsymbol{\nu} \boldsymbol{F} X$ : $\boldsymbol{G F} X \rightarrow \boldsymbol{G}^{\prime} \boldsymbol{F}^{\prime} X$ instead of $\boldsymbol{G}^{\prime}\left(\boldsymbol{\mu}_{X}\right) \circ \boldsymbol{\nu}_{\boldsymbol{F}(X)}$. In equalities of natural transformations we shall omit the object $X$ in our formulae.

Inspired by the diagrammatic computation in a 2-category (in particular CAT), we shall use a graphical representation of morphisms in a category. For functors $\boldsymbol{F}_{1}, \ldots, \boldsymbol{F}_{n}, \boldsymbol{G}_{1}, \ldots, \boldsymbol{G}_{m}$, which can be composed to $\boldsymbol{F}_{1} \boldsymbol{F}_{2} \ldots \boldsymbol{F}_{n}: \mathcal{D}_{1} \rightarrow \mathcal{C}$ and $\boldsymbol{G}_{1} \boldsymbol{G}_{2} \ldots \boldsymbol{G}_{m}: \mathcal{D}_{2} \rightarrow \mathcal{C}$, and objects $X$ in $\mathcal{D}_{1}$ and $Y$ in $\mathcal{D}_{2}$, a morphism $f: \boldsymbol{F}_{1} \boldsymbol{F}_{2} \ldots \boldsymbol{F}_{n} X \rightarrow \boldsymbol{G}_{1} \boldsymbol{G}_{2} \ldots \boldsymbol{G}_{m} Y$ will be represented vertically, with the domain up, as in Figure 1(a). Furthermore, for a functor $\boldsymbol{T}: \mathcal{C} \rightarrow \mathcal{C}^{\prime}$, the morphism $\boldsymbol{T} f$ will be drawn as in (b). Keeping the notation from the first paragraph of this section, the picture representing $\boldsymbol{\mu} G X$ is shown in diagram (c). The composition $g \circ f$ of the morphisms $f: X \rightarrow Y$ and $g: Y \rightarrow Z$ will be represented as in diagram (d). For the multiplication $\boldsymbol{m}_{\boldsymbol{T}}$ and the unit $\boldsymbol{u}_{\boldsymbol{T}}$ of a monad $\boldsymbol{T}$ on $\mathcal{C}$ (see Definition 1.1), and an object $X$ in $\mathcal{C}$, to draw $\boldsymbol{m}_{\boldsymbol{T}} X$ and $\boldsymbol{u}_{\boldsymbol{T}} X$ we shall use the diagrams (e) and (f), while for a distributive law $\boldsymbol{t}: \boldsymbol{R} \boldsymbol{T} \rightarrow \boldsymbol{T} \boldsymbol{R}$ (see Definition 1.3) $\boldsymbol{t} X$ will be drawn as in the picture (g). If $\boldsymbol{t}$ is invertible, the representation of $\boldsymbol{t}^{-1} X$ is shown in diagram (h). For simplifying diagrams containing only natural transformations, we shall always omit the

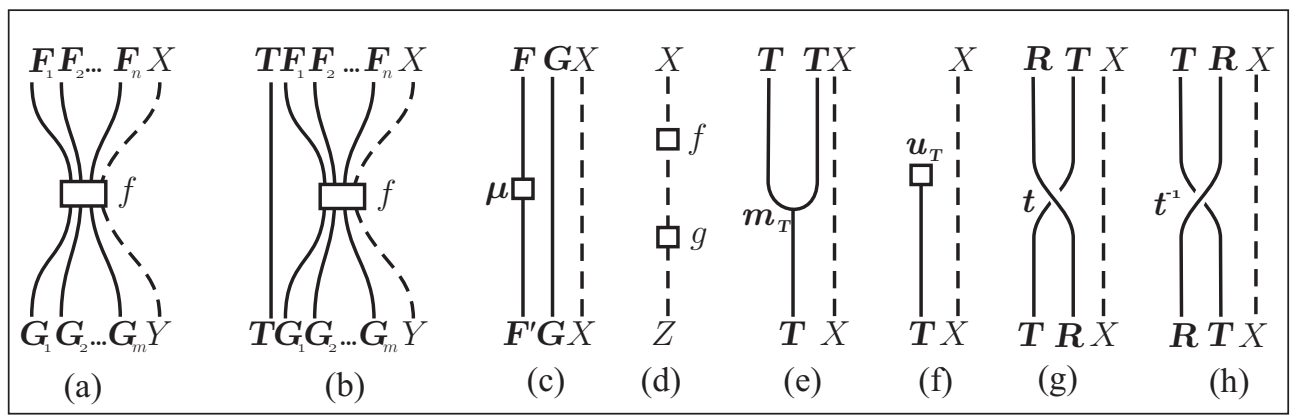

FIGURE 1. Diagrammatic representation of morphisms in a category

last string that corresponds to an object in the category. That is, we work with diagrams in CAT whenever it is possible.

We shall use the following method to perform computations with such diagrams. In view of associativity of composition, any diagram, representing a well-defined composition of morphisms, can be thought of as a tower with several layers. Any part of the diagram, corresponding to a layer, can be substituted with any other equivalent representation of it. Usually, equivalent representations are obtained from formulas that define the notions that we deal with, or equations that have been previously proved.

1.B. Monads and distributive laws. Monads represent the main ingredient in our approach to cyclic (co)homology. The definition of monads traces back to Godement's book [Go], where they are called "standard constructions". In the literature they are also called "triples", see for example [EM]. 
Definition 1.1. A monad on a category $\mathcal{C}$ is a triple $\left(\boldsymbol{T}, \boldsymbol{m}_{\boldsymbol{T}}, \boldsymbol{u}_{\boldsymbol{T}}\right)$, where $\boldsymbol{T}: \mathcal{C} \rightarrow \mathcal{C}$ is a functor, $\boldsymbol{m}_{\boldsymbol{T}}: \boldsymbol{T}^{2} \rightarrow \boldsymbol{T}$ and $\boldsymbol{u}_{\boldsymbol{T}}: \boldsymbol{I} \boldsymbol{d}_{\mathcal{C}} \rightarrow \boldsymbol{T}$ are natural transformations such that the first two diagrams in Figure 2, expressing associativity and unitality, are commutative. We call $\boldsymbol{m}_{\boldsymbol{T}}$ and $\boldsymbol{u}_{\boldsymbol{T}}$ the

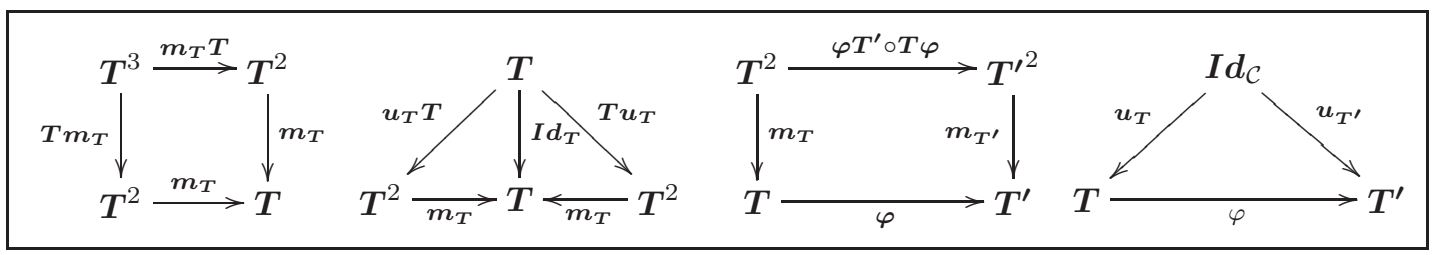

Figure 2. Monads and morphisms of monads.

multiplication and the unit of the monad $\boldsymbol{T}$, respectively.

For two monads $\left(\boldsymbol{T}, \boldsymbol{m}_{\boldsymbol{T}}, \boldsymbol{u}_{\boldsymbol{T}}\right)$ and $\left(\boldsymbol{T}^{\prime}, \boldsymbol{m}_{\boldsymbol{T}^{\prime}}, \boldsymbol{u}_{\boldsymbol{T}^{\prime}}\right)$ on $\mathcal{C}$, we say that a natural transformation $\varphi: \boldsymbol{T} \rightarrow \boldsymbol{T}^{\prime}$ is a morphism of monads if the last two diagrams in Figure 2 are commutative.

ExAmPle 1.2. Let $(\mathcal{C}, \otimes, \boldsymbol{a}, \boldsymbol{l}, \boldsymbol{r}, \mathbf{1})$ be a monoidal category with unit object $\mathbf{1}$, associativity constraint $\boldsymbol{a}$ and unit constraints $\boldsymbol{l}, \boldsymbol{r}$. For details about monoidal categories the reader is referred to [Kass, Chapter XI]. An algebra in $\mathcal{C}$ is a triple $\left(T, m_{T}, u_{T}\right)$ such that $m_{T}: T \otimes T \rightarrow T$ defines an associative multiplication on $T$ with unit $u_{T}: \mathbf{1} \rightarrow T$. To such an algebra one can associate two monads $\boldsymbol{T}_{\boldsymbol{l}}:=T \otimes(-)$ and $\boldsymbol{T}_{\boldsymbol{r}}:=(-) \otimes T$ on $\mathcal{C}$. The multiplication $\boldsymbol{m}_{\boldsymbol{T}_{l}}$ and the unit $\boldsymbol{u}_{\boldsymbol{T}_{l}}$ of $\boldsymbol{T}_{\boldsymbol{l}}$ are given by

$$
\boldsymbol{m}_{\boldsymbol{T}_{l}} X:=\left(m_{T} \otimes X\right) \circ \boldsymbol{a}_{T, T, X}^{-1} \quad \text { and } \quad \boldsymbol{u}_{\boldsymbol{T}_{l}} X:=\left(u_{T} \otimes X\right) \circ \boldsymbol{l}_{X}^{-1},
$$

for every $X$ in $\mathcal{C}$. Analogously, for $X$ in $\mathcal{C}, \boldsymbol{m}_{\boldsymbol{T}_{r}} X$ and $\boldsymbol{u}_{\boldsymbol{T}_{r}} X$ are defined by

$$
\boldsymbol{m}_{\boldsymbol{T}_{\boldsymbol{r}}} X:=\left(X \otimes m_{T}\right) \circ \boldsymbol{a}_{X, T, T} \quad \text { and } \quad \boldsymbol{u}_{\boldsymbol{T}_{\boldsymbol{r}}} X:=\left(X \otimes u_{T}\right) \circ \boldsymbol{r}_{X}^{-1} .
$$

A homomorphism $\varphi: T \rightarrow T^{\prime}$ of algebras in $\mathcal{C}$ induces monad morphisms $\varphi_{l}: \boldsymbol{T}_{l} \rightarrow \boldsymbol{T}_{l}^{\prime}$ and $\varphi_{r}: \boldsymbol{T}_{\boldsymbol{r}} \rightarrow \boldsymbol{T}_{r}^{\prime}$. For example, $\varphi_{l} X:=\varphi \otimes \mathrm{id}_{X}$, for any object $X$ in $\mathcal{C}$.

A particular case of these constructions, which is very important for our work, is obtained when we take $\mathcal{C}$ to be the category $R$-Mod- $R$ of bimodules over an ordinary $\mathbb{K}$-algebra $R$ (i.e $R$ is an algebra in the category of $\mathbb{K}$-modules, where $\mathbb{K}$ is a commutative ring). The category $R$-Mod- $R$ is monoidal with respect to the $R$-module tensor product $\otimes_{R}$. Unit object is $R$. An algebra in $R$-Mod- $R$ is called an $R$-ring. $R$-rings $\left(T, m_{T}, \varphi\right)$ are in bijective correspondence with $\mathbb{K}$-algebra maps $\varphi: R \rightarrow T$. Indeed, for an algebra $\left(T, m_{T}, \varphi\right)$ in $R$-Mod- $R$, composition of the canonical epimorphism $T \otimes_{\mathbb{K}} T \rightarrow T \otimes_{R} T$ with $m_{T}: T \otimes_{R} T \rightarrow T$ defines a $\mathbb{K}$-algebra structure on $T$ such that $\varphi$ is a $\mathbb{K}$-algebra homomorphism. Conversely, via a $\mathbb{K}$-algebra homomorphism $\varphi: R \rightarrow T, T$ becomes an $R$-bimodule. Multiplication of $T$ induces a morphism $m_{T}$ from $T \otimes_{R} T$ to $T$, which makes $T$ an associative algebra in $R$-Mod- $R$. The unit of $T$ is $\varphi$. (With a slight abuse of notation, we denote both multiplication maps $T \otimes_{R} T \rightarrow T$ and $T \otimes_{\mathbb{K}} T \rightarrow T$ by the same symbol $m_{T}$.) Consequently, a $\mathbb{K}$-algebra homomorphism $\varphi: R \rightarrow T$ defines two monads $T \otimes_{R}(-)$ and $(-) \otimes_{R} T$ on $R$-Mod- $R$.

Distributive laws were introduced by J. Beck [Be]. They give a way to compose two monads in order to obtain a monad.

Definition 1.3. A distributive law between two monads $\left(\boldsymbol{R}, \boldsymbol{m}_{\boldsymbol{R}}, \boldsymbol{u}_{\boldsymbol{R}}\right)$ and $\left(\boldsymbol{T}, \boldsymbol{m}_{\boldsymbol{T}}, \boldsymbol{u}_{\boldsymbol{T}}\right)$ is a natural transformation $\boldsymbol{t}: \boldsymbol{R} \boldsymbol{T} \rightarrow \boldsymbol{T} \boldsymbol{R}$ satisfying the four conditions in Figure 3.

REMARK 1.4. Since we are using for the first time the diagrammatic representation of morphisms, let us write out explicitly the first and the third relations in Figure 3. They read as

$$
t \circ m_{R} T=T m_{R} \circ t R \circ R t, \quad t \circ u_{R} T=T u_{R} .
$$




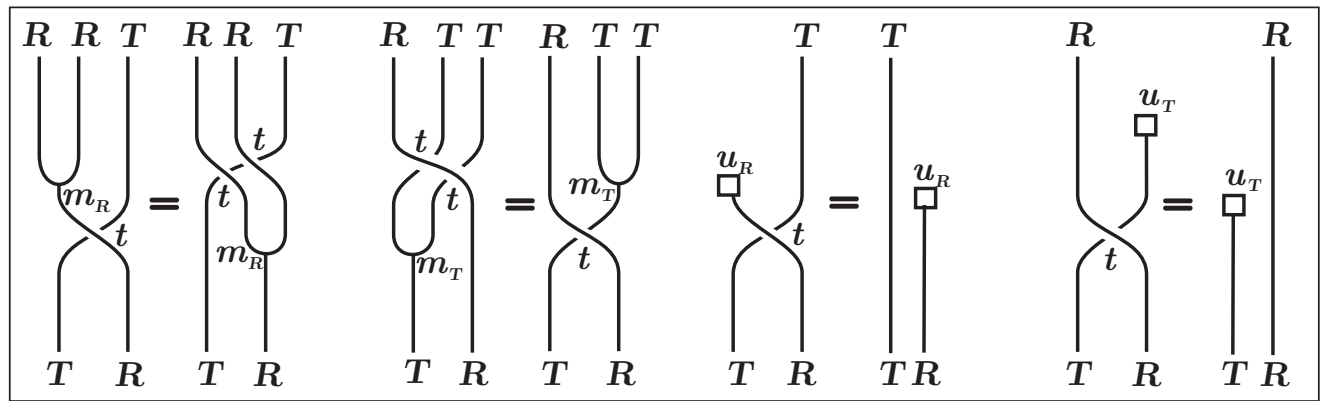

Figure 3. The definition of distributive laws.

EXAMPLE 1.5. Let $T$ be an algebra in a monoidal category as in Example 1.2. Keeping the the notation from Example 1.2, the natural transformation $\boldsymbol{t}: \boldsymbol{T}_{\boldsymbol{r}} \boldsymbol{T}_{\boldsymbol{l}} \rightarrow \boldsymbol{T}_{\boldsymbol{l}} \boldsymbol{T}_{\boldsymbol{r}}$, given by

$$
\boldsymbol{t} X:=\boldsymbol{a}_{T, X, T}
$$

for any object $X$ in $\mathcal{C}$, is a distributive law. Note that the first equality in Figure 3 follows by the Pentagon Axiom [Kass, p. 282, Diagram (2.6)], applied to the quadruple $(T, X, T, T)$. Similarly, by applying The Pentagon Axiom for $(T, T, X, T)$ we deduce the second equality in the definition of distributive laws. The fourth equality in Figure 3 is a consequence of $\boldsymbol{l}_{X \otimes T} \circ \boldsymbol{a}_{\mathbf{1}, X, T}=\boldsymbol{l}_{X} \otimes T$, see [Kass, Lemma XI.2.2]. The other relation in the above cited lemma can be used to prove that the third equality in Figure 3 holds too.

EXAMPLE 1.6. Let $\mathcal{C}$ be a braided monoidal category with braiding $\boldsymbol{c}_{X, Y}: X \otimes Y \rightarrow Y \otimes X$. For the definition and properties of braided monoidal categories see [Kass, Chapter XIII]. If $R$ and $T$ are algebras in $\mathcal{C}$ then

$$
\boldsymbol{t} X:=\boldsymbol{a}_{T, R, X} \circ\left(\boldsymbol{c}_{R, T} \otimes X\right) \circ \boldsymbol{a}_{R, T, X}^{-1}
$$

defines a distributive law $\boldsymbol{t}: \boldsymbol{R}_{\boldsymbol{l}} \boldsymbol{T}_{\boldsymbol{l}} \rightarrow \boldsymbol{T}_{\boldsymbol{l}} \boldsymbol{R}_{\boldsymbol{l}}$, where $\boldsymbol{R}_{\boldsymbol{l}}$ and $\boldsymbol{T}_{\boldsymbol{l}}$ are constructed as in Example 1.2. Obviously, $\boldsymbol{t}^{-1}: \boldsymbol{T}_{l} \boldsymbol{R}_{\boldsymbol{l}} \rightarrow \boldsymbol{R}_{l} \boldsymbol{T}_{\boldsymbol{l}}$ is also a distributive law.

1.C. Admissible septuples and transposition maps. The main result. In this section we introduce admissible septuples and transposition morphisms of them. We show that to these data one associates functorially para-cocyclic objects. Our aim is twofold. On one hand, in this way we obtain a very general but at the same time technically very simple framework. In particular, it can be used to associate para-cocyclic objects to (co)module algebras of bialgebroids, cf. Section 2.A. On the other hand, the resulting setting will be easily dualized to describe in Section 2.B the situation dual to that in Section 2.A, i.e. the (para-)cyclic objects associated to (co)module corings of bialgebroids.

Definition 1.7. An admissible septuple $\mathcal{S}:=\left(\mathcal{M}, \mathcal{C}, \boldsymbol{T}_{\boldsymbol{l}}, \boldsymbol{T}_{\boldsymbol{r}}, \boldsymbol{\Pi}, \boldsymbol{t}, \boldsymbol{i}\right)$ is defined by the following data:

- Two categories $\mathcal{M}$ and $\mathcal{C}$;

- Two monads $\boldsymbol{T}_{\boldsymbol{l}}$ and $\boldsymbol{T}_{\boldsymbol{r}}$ on $\mathcal{M}$;

- A functor $\Pi: \mathcal{M} \rightarrow \mathcal{C}$;

- A distributive law $t: T_{r} T_{l} \rightarrow T_{l} T_{r}$

- A natural transformation $i: \Pi T_{l} \rightarrow \Pi T_{r}$.

These data are assumed to satisfy the relations

$$
i \circ \Pi u_{T_{l}}=\Pi u_{T_{r}} \quad \text { and } \quad i \circ \Pi m_{T_{l}}=\Pi m_{T_{r}} \circ i T_{r} \circ \Pi t \circ i T_{l} .
$$

Examples of admissible septuples will be given in Section 1.D, where also several applications of the main result of this section, Theorem 1.10, will be indicated.

By [We, p. 281], to every monad $\boldsymbol{T}_{\boldsymbol{l}}: \mathcal{M} \rightarrow \mathcal{M}$ and object $X$ in $\mathcal{M}$ one can associate a cosimplicial object of components $\boldsymbol{T}_{l}^{n+1} X$ in $\mathcal{M}$. Thus in particular an admissible septuple $\mathcal{S}$ in 
Definition 1.7 determines a cosimplicial object in $\mathcal{M}$. It can be transported to $\mathcal{C}$ via the functor $\Pi: \mathcal{M} \rightarrow \mathcal{C}$ in Definition 1.7. The resulting cosimplex in $\mathcal{C}$ will be denoted by $Z^{*}(\mathcal{S}, X)$. By construction, $Z^{n}(\mathcal{S}, X)=\boldsymbol{\Pi} \boldsymbol{T}_{l}{ }^{n+1} X$ and, for every $k \in\{0, \ldots, n\}$, the coface maps $d_{k}: \boldsymbol{\Pi} \boldsymbol{T}_{l}^{n} X \rightarrow$ $\boldsymbol{\Pi} \boldsymbol{T}_{l}{ }^{n+1} X$ and the codegeneracy maps $s_{k}: \boldsymbol{\Pi} \boldsymbol{T}_{l}{ }^{n+2} X \rightarrow \boldsymbol{\Pi} \boldsymbol{T}_{l}{ }^{n+1} X$ are given by

$$
d_{k}:=\boldsymbol{\Pi} \boldsymbol{T}_{l}^{k} \boldsymbol{u}_{\boldsymbol{T}_{l}} \boldsymbol{T}_{\boldsymbol{l}}^{n-k} X, \quad s_{k}:=\boldsymbol{\Pi} \boldsymbol{T}_{l}^{k} \boldsymbol{m}_{\boldsymbol{T}_{\boldsymbol{l}}} \boldsymbol{T}_{\boldsymbol{l}}{ }^{n-k} X .
$$

Our aim is to construct a category $\mathcal{W}_{\mathcal{S}}$ such that $Z^{*}(\mathcal{S},-)$ can be regarded as a functor from $\mathcal{W}_{\mathcal{S}}$ to the category of para-cocyclic objects in $\mathcal{C}$. Observe that, for an admissible septuple $\mathcal{S}$ in Definition 1.7, the distributive law $\boldsymbol{t}$ is lifted to a natural transformation $\boldsymbol{t}_{n}: \boldsymbol{\Pi} \boldsymbol{T}_{\boldsymbol{r}} \boldsymbol{T}_{l}{ }^{n} \rightarrow \boldsymbol{\Pi} \boldsymbol{T}_{l}{ }^{n} \boldsymbol{T}_{\boldsymbol{r}}$,

$$
\boldsymbol{t}_{n}:=\boldsymbol{\Pi} \boldsymbol{T}_{l}^{n-1} \boldsymbol{t} \circ \boldsymbol{\Pi} \boldsymbol{T}_{l}^{n-2} \boldsymbol{t} \boldsymbol{T}_{l} \circ \cdots \circ \boldsymbol{\Pi} \boldsymbol{T}_{l} \boldsymbol{t} \boldsymbol{T}_{l}{ }^{n-2} \circ \Pi \boldsymbol{t} \boldsymbol{T}_{l}{ }^{n-1} .
$$

Definition 1.8. Let $\mathcal{S}:=\left(\mathcal{M}, \mathcal{C}, \boldsymbol{T}_{\boldsymbol{l}}, \boldsymbol{T}_{\boldsymbol{r}}, \boldsymbol{\Pi}, \boldsymbol{t}, \boldsymbol{i}\right)$ be an admissible septuple. We say that an arrow $w: \boldsymbol{T}_{\boldsymbol{r}} X \rightarrow \boldsymbol{T}_{l} X$ in $\mathcal{M}$ is a transposition morphism with respect to $\mathcal{S}$ if

$$
w \circ \boldsymbol{u}_{\boldsymbol{T}_{\boldsymbol{r}}} X=\boldsymbol{u}_{\boldsymbol{T}_{l}} X \quad \text { and } \quad w \circ \boldsymbol{m}_{\boldsymbol{T}_{r}} X=\boldsymbol{m}_{\boldsymbol{T}_{l}} X \circ \boldsymbol{T}_{l} w \circ \boldsymbol{t} X \circ \boldsymbol{T}_{\boldsymbol{r}} w .
$$

The category of pairs $(X, w)$, with $w: \boldsymbol{T}_{\boldsymbol{r}} X \rightarrow \boldsymbol{T}_{l} X$ a transposition morphism of $\mathcal{S}$, will be denoted by $\mathcal{W}_{\mathcal{S}}$. A morphism from $(X, w)$ to $\left(X^{\prime}, w^{\prime}\right)$ is an arrow $f: X \rightarrow X^{\prime}$ in $\mathcal{M}$ such that $\boldsymbol{T}_{l} f \circ w=w^{\prime} \circ \boldsymbol{T}_{r} f$.

Morphisms $w: \boldsymbol{T}_{\boldsymbol{r}} X \rightarrow \boldsymbol{T}_{l} X$ satisfying (1.5), for a distributive law $\boldsymbol{t}: \boldsymbol{T}_{\boldsymbol{r}} \boldsymbol{T}_{\boldsymbol{l}} \rightarrow \boldsymbol{T}_{l} \boldsymbol{T}_{\boldsymbol{r}}$, were termed $\boldsymbol{t}$-algebras in [Burr]. Based on [Burr, Proposition I.1.1], transposition morphisms can be characterized as in Proposition 1.9 below. Recall that a module of a monad $\left(\boldsymbol{T}, \boldsymbol{m}_{\boldsymbol{T}}, \boldsymbol{u}_{\boldsymbol{T}}\right)$ on a category $\mathcal{M}$ is a pair $(Y, \varrho)$, consisting of an object $Y$ and a morphism $\varrho: T Y \rightarrow Y$ in $\mathcal{M}$, such that $\varrho \circ \boldsymbol{T} \varrho=\varrho \circ \boldsymbol{m}_{\boldsymbol{T}} Y$ and $\varrho \circ \boldsymbol{u}_{\boldsymbol{T}} Y=\mathrm{Id}_{Y}$ (i.e. $\varrho$ is associative and unital). A morphism of $\boldsymbol{T}$-modules $(Y, \varrho) \rightarrow\left(Y^{\prime}, \varrho^{\prime}\right)$ is a morphism $f: Y \rightarrow Y^{\prime}$ in $\mathcal{M}$, such that $f \circ \varrho=\varrho^{\prime} \circ \boldsymbol{T} f$.

Proposition 1.9. Consider an admissible septuple $\mathcal{S}:=\left(\mathcal{M}, \mathcal{C}, \boldsymbol{T}_{\boldsymbol{l}}, \boldsymbol{T}_{\boldsymbol{r}}, \boldsymbol{\Pi}, \boldsymbol{t}, \boldsymbol{i}\right)$. There is a bijective correspondence between objects $(X, w)$ in the category $\mathcal{W}_{\mathcal{S}}$ and $\boldsymbol{T}_{\boldsymbol{r}}$-modules of the form $\left(\boldsymbol{T}_{l} X, \varrho\right)$, satisfying

$$
\boldsymbol{m}_{\boldsymbol{T}_{l}} X \circ \boldsymbol{T}_{l} \varrho \circ \boldsymbol{t} \boldsymbol{T}_{\boldsymbol{l}} X=\varrho \circ \boldsymbol{T}_{\boldsymbol{r}} \boldsymbol{m}_{\boldsymbol{T}_{l}} X .
$$

Moreover, a morphism $f: X \rightarrow X^{\prime}$ in $\mathcal{M}$ is a morphism in $\mathcal{W}_{\mathcal{S}}$ if and only if $\boldsymbol{T}_{l} f$ is a $\boldsymbol{T}_{\boldsymbol{r}}$-module morphism.

Proof. Similarly to the proof of [Burr, Proposition I.1.1] one checks that, for an object $(X, w)$ in $\mathcal{W}_{\mathcal{S}}$, an associative and unital $\boldsymbol{T}_{\boldsymbol{r}}$-action on $\boldsymbol{T}_{\boldsymbol{l}} X$ satisfying (1.6) is given by $\varrho_{w}:=\boldsymbol{m}_{\boldsymbol{T}_{l}} X \circ$ $\boldsymbol{T}_{l} w \circ \boldsymbol{t} X: \boldsymbol{T}_{r} \boldsymbol{T}_{l} X \rightarrow \boldsymbol{T}_{l} X$. Conversely, note that for a $\boldsymbol{T}_{r}$-module $\left(\boldsymbol{T}_{l} X, \varrho\right)$, (1.6) is equivalent to $\varrho=\boldsymbol{m}_{\boldsymbol{T}_{l}} X \circ \boldsymbol{T}_{l} \varrho \circ \boldsymbol{T}_{l} \boldsymbol{T}_{\boldsymbol{r}} \boldsymbol{u}_{\boldsymbol{T}_{l}} X \circ \boldsymbol{t} X$. With this identity at hand, the pair $\left(X, w_{\varrho}:=\varrho \circ \boldsymbol{T}_{\boldsymbol{r}} \boldsymbol{u}_{\boldsymbol{T}_{l}} X\right)$ is checked to be an object in $\mathcal{W}_{\mathcal{S}}$. A straightforward computation shows that the two constructions are mutual inverses. A morphism $\boldsymbol{T}_{l} f$ is a morphism of $\boldsymbol{T}_{\boldsymbol{r}}$-modules $\left(\boldsymbol{T}_{l} X, \varrho_{w}\right) \rightarrow\left(\boldsymbol{T}_{l} X^{\prime}, \varrho_{w^{\prime}}\right)$ if

$$
\boldsymbol{m}_{\boldsymbol{T}_{l}} X^{\prime} \circ \boldsymbol{T}_{\boldsymbol{l}} w^{\prime} \circ \boldsymbol{T}_{\boldsymbol{l}} \boldsymbol{T}_{\boldsymbol{r}} f \circ \boldsymbol{t} X=\boldsymbol{m}_{\boldsymbol{T}_{l}} X^{\prime} \circ \boldsymbol{T}_{l} \boldsymbol{T}_{l} f \circ \boldsymbol{T}_{\boldsymbol{l}} w \circ \boldsymbol{t} X .
$$

If $f$ is a morphism in $\mathcal{W}_{\mathcal{S}}$ then (1.7) obviously holds. In order to prove the converse implication, compose both sides of (1.7) with $\boldsymbol{T}_{\boldsymbol{r}} \boldsymbol{u}_{\boldsymbol{T}_{l}} X$ on the right.

TheOrem 1.10. Consider an admissible septuple $\mathcal{S}$ and a transposition map $w: \boldsymbol{T}_{\boldsymbol{r}} X \rightarrow \boldsymbol{T}_{\boldsymbol{l}} X$ in $\mathcal{W}_{\mathcal{S}}$. The cosimplicial object $Z^{*}(\mathcal{S}, X)$ is para-cocyclic with respect to

$$
w_{n}:=\boldsymbol{\Pi} \boldsymbol{T}_{l}^{n} w \circ \boldsymbol{t}_{n} X \circ \boldsymbol{i} \boldsymbol{T}_{l}^{n} X .
$$

We shall denote this para-cocyclic object by $Z^{*}(\mathcal{S}, w)$. For a morphism $f:(X, w) \rightarrow\left(X^{\prime}, w^{\prime}\right)$ in $\mathcal{W}_{\mathcal{S}}$, the morphisms $\boldsymbol{\Pi} \boldsymbol{T}_{l}{ }^{n+1} f: Z^{n}(\mathcal{S}, w) \rightarrow Z^{n}\left(\mathcal{S}, w^{\prime}\right)$ determine a morphism of para-cocyclic objects.

Proof. In Figure 4 we show that the morphism (1.8) is compatible with the coface maps, that is

$$
w_{n} \circ d_{0}=d_{n} \quad \text { and } \quad w_{n} \circ d_{k}=d_{k-1} \circ w_{n-1}
$$

for any $k \in\{1, \ldots, n\}$. The proof of the first equation is given in three steps in the left picture. To simplify the diagrams, we draw the $n$ strings representing $\boldsymbol{T}_{l}^{n}$ as a black stripe. For the first 
equality we used the compatibility between $\boldsymbol{i}$ and the unit of $\boldsymbol{T}_{\boldsymbol{l}}$, that is the first equation in (1.2). Next we applied $n$ times the compatibility relation between the distributive law $\boldsymbol{t}$ and the unit of $\boldsymbol{T}_{\boldsymbol{r}}$, i.e. the third equality in Figure 3. The first relation in (1.5) implies the third equality. The
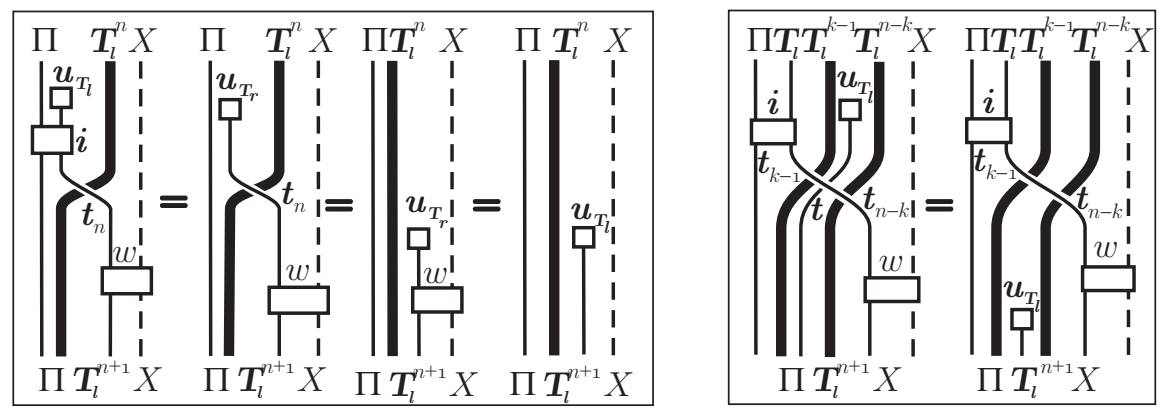

Figure 4. The proof of the relations (1.9).

second relation in (1.9) follows in a similar way, as it is shown in the right picture in Figure 4. Note that the leftmost black stripe represents $\boldsymbol{T}_{l}{ }^{k-1}$ and the other one represents $\boldsymbol{T}_{l}{ }^{n-k}$. Since $\boldsymbol{u}_{\boldsymbol{T}_{l}}$ is a natural transformation, the box representing it can be pushed down along the string until it meets the crossing $\boldsymbol{t}$. By the fourth identity in Figure 3, one can push $\boldsymbol{u}_{T_{l}}$ under the string in the crossing. To conclude the proof of this equality, we use once again that $\boldsymbol{u}_{\boldsymbol{T}_{l}}$ is a natural transformation to move it to the bottom of the diagram.

Next we prove that the morphism (1.8) and the codegeneracy maps are compatible too, that is

$$
w_{n} \circ s_{0}=s_{n} \circ\left(w_{n+1}\right)^{2} \quad \text { and } \quad w_{n} \circ s_{k}=s_{k-1} \circ w_{n+1}
$$

for any $k \in\{1, \ldots, n\}$. The proof of the first relation can be found in the left picture in Figure 5 . As before, the black stripe represents $\boldsymbol{T}_{l}{ }^{n}$. The morphisms corresponding to the first two diagrams are equal in view of the second equation in (1.2). By applying $n$ times the first identity in Figure 3, it follows that the second and the third diagrams represent the same morphism. Taking into account the second relation in (1.5) we got the penultimate equality, while for the last one we used that $\boldsymbol{i}$ is a natural transformation.
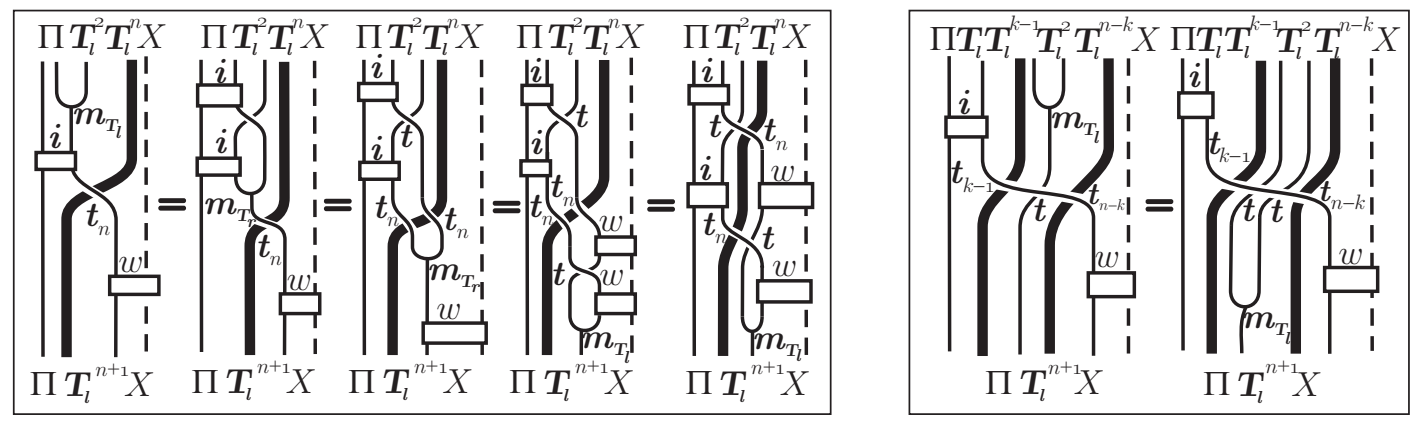

Figure 5. The proof of the relations (1.10).

The other relation in (1.10) immediately follows by the second identity in Figure 3 and the fact $\boldsymbol{m}_{\boldsymbol{T}_{l}}$ is natural (see the second picture in Figure 5).

Since the coface and codegeneracy morphisms (1.3) are defined in terms of natural transformations, the morphisms $\boldsymbol{\Pi} \boldsymbol{T}_{l}{ }^{n+1} f: Z^{n}(\mathcal{S}, X) \rightarrow Z^{n}\left(\mathcal{S}, X^{\prime}\right)$ determine a morphism $Z^{*}(\mathcal{S}, f)$ : $Z^{*}(\mathcal{S}, X) \rightarrow Z^{*}\left(\mathcal{S}, X^{\prime}\right)$ of cosimplicial objects, for any morphism $f: X \rightarrow X^{\prime}$ in $\mathcal{M}$. It follows 
immediately from the definition of a morphism in $\mathcal{W}_{\mathcal{S}}$ that if $f:(X, w) \rightarrow\left(X^{\prime}, w^{\prime}\right)$ is a morphism in $\mathcal{W}_{\mathcal{S}}$ then $Z^{*}(\mathcal{S}, f)$ is a morphism of para-cocyclic objects $Z^{*}(\mathcal{S}, w) \rightarrow Z^{*}\left(\mathcal{S}, w^{\prime}\right)$.

Corollary 1.11. Let $\mathcal{S}$ be an admissible septuple as in Definition 1.7 and let $w: \boldsymbol{T}_{\boldsymbol{r}} X \rightarrow \boldsymbol{T}_{\boldsymbol{l}} X$ be a transposition morphism in $\mathcal{W}_{\mathcal{S}}$. Consider the corresponding para-cocyclic morphism $w_{n}$ in (1.8). If the coequalizer

$$
Z^{n}(\mathcal{S}, w) \underset{\operatorname{Id}_{Z^{n}}(\mathcal{S}, w)}{\stackrel{\left(w_{n}\right)^{n+1}}{\longrightarrow}} Z^{n}(\mathcal{S}, w) \longrightarrow \widehat{Z}^{n}(\mathcal{S}, w)
$$

exists in $\mathcal{C}$, for every non-negative integer $n$, then it defines a cocyclic cosimplex $\widehat{Z}^{*}(\mathcal{S}, w)$.

Proof. Let $k \in\{0, \ldots, n\}$. It follows by (1.9) that $d_{k}$ satisfies $d_{k} \circ\left(w_{n}\right)^{n+1}=\left(w_{n+1}\right)^{n+2} \circ d_{k}$. Similarly, by (1.10), the codegeneracy morphism $s_{k}$ satisfies $s_{k} \circ\left(w_{n}\right)^{n+1}=\left(w_{n-1}\right)^{n} \circ s_{k}$. Hence $d_{k}$ and $s_{k}$ determine coface morphisms $\widehat{d}_{k}$ and codegeneracy morphisms $\widehat{s}_{k}$ on $\widehat{Z}^{n}(\mathcal{S}, X)$. Together with the projection $\widehat{w}_{n}$ of $w_{n}$ onto $\widehat{Z}^{n}(\mathcal{S}, X)$ they define a cocyclic object $\left(\widehat{Z}^{*}(\mathcal{S}, w), \widehat{d}^{*}, \widehat{s}^{*}, \widehat{w}^{*}\right)$.

1.D. Examples of admissible septuples and transposition maps. Applications. In this section we shall apply Theorem 1.10 to several examples of admissible septuples. In this way we shall show that the most known (co)cyclic objects in the literature can be obtained as direct applications of the result obtained in Section 1.C.

A functorial construction of a para-cyclic object in a category of endofunctors, of a somewhat similar flavour to that in Theorem 1.10, was proposed in [Šk $]$. The following example is its dual version.

EXAmple 1.12. Let $\boldsymbol{T}=(\boldsymbol{T}, \boldsymbol{m}, \boldsymbol{u})$ be a monad on a category $\mathcal{M}$ and $\boldsymbol{t}: \boldsymbol{T} \boldsymbol{T} \rightarrow \boldsymbol{T} \boldsymbol{T}$ be a distributive law. Assume that $\boldsymbol{t}$ satisfies the Yang-Baxter relation

$$
t T \circ T t \circ t T=T t \circ t T \circ T t
$$

of natural transformations $\boldsymbol{T} \boldsymbol{T} \boldsymbol{T} \rightarrow \boldsymbol{T} \boldsymbol{T} \boldsymbol{T}$, and $\boldsymbol{m} \circ \boldsymbol{t} \circ \boldsymbol{t}=\boldsymbol{m}$. As a consequence of (1.11), also $\boldsymbol{T}^{0}=(\boldsymbol{T}, \boldsymbol{m} \circ \boldsymbol{t}, \boldsymbol{u})$ is a monad, and $\boldsymbol{t}$ can be regarded as a distributive law $\boldsymbol{T}^{\mathbf{0}} \boldsymbol{T} \rightarrow \boldsymbol{T} \boldsymbol{T}^{\mathbf{0}}$. Furthermore, the datum $\mathcal{S}:=\left(\mathcal{M}, \mathcal{M}, \boldsymbol{T}, \boldsymbol{T}^{\mathbf{0}}, \operatorname{Id}_{\mathcal{M}}, \boldsymbol{t}, \operatorname{Id}_{\boldsymbol{T}}\right)$ (where $\operatorname{Id}_{\mathcal{M}}$ denotes the identity functor $\mathcal{M} \rightarrow \mathcal{M}$ and $\operatorname{Id}_{\boldsymbol{T}}$ is the identity natural transformation $\left.\boldsymbol{T} \rightarrow \boldsymbol{T}\right)$ is an admissible septuple. For any object $X$ in $\mathcal{M}$, the identity morphism $\operatorname{Id}_{\boldsymbol{T} X}$ is a transposition morphism. The corresponding para-cocyclic morphism is $\boldsymbol{t}_{n}$ in (1.4).

The simplest example of an admissible septuple can be obtained by starting with a morphism $\varphi: R \rightarrow T$ of $\mathbb{K}$-algebras. As in Example 1.2, we define two monads on the category $\mathcal{M}:=R$ Mod- $R$ by $\boldsymbol{T}_{l}:=T \otimes_{R}(-)$ and $\boldsymbol{T}_{\boldsymbol{r}}:=(-) \otimes_{R} T$. The category $\mathcal{C}$ is, by definition, the category Mod- $\mathbb{K}$ of $\mathbb{K}$-modules. The functor $\boldsymbol{\Pi}$ is constructed below.

Definition 1.13. On the objects $X \in R$-Mod- $R$, the functor $\Pi: R$-Mod- $R \rightarrow$ Mod- $\mathbb{K}$ is defined as a coequalizer

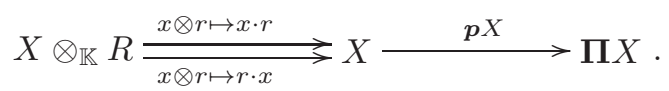

For a morphism $f: X \rightarrow Y$ of $R$-bimodules, $\Pi f$ is the unique $\mathbb{K}$-linear map such that $p Y \circ f=$ $\Pi f \circ p X$. Hence $p$ can be interpreted as a natural epimorphism from the forgetful functor $U$ : $R$-Mod- $R \rightarrow$ Mod- $\mathbb{K}$ to $\Pi$.

Remark 1.14. An $R$-bimodule $X$ can be considered as a left or right module for the enveloping algebra $R^{e}:=R \otimes_{\mathbb{K}} R^{o p}$ of $R$. In terms of the functor $\Pi$ in Definition 1.13, the cyclic tensor product $X \widehat{\otimes}_{R} Y$ of two $R$-bimodules $X$ and $Y$ is defined by $X \widehat{\otimes}_{R} Y:=\Pi\left(X \otimes_{R} Y\right) \cong X \otimes_{R^{e}} Y$. With this interpretation in mind, the $\mathbb{K}$-module $\Pi X \cong R \otimes_{R^{e}} X$ can be seen as the cyclic tensor product of $R$ and $X$. For $R$-bimodules $X_{1}, \ldots, X_{n}$, the $n$-fold cyclic module tensor product is defined as

$$
X_{1} \widehat{\otimes}_{R} \ldots \widehat{\otimes}_{R} X_{n}:=\Pi\left(X_{1} \otimes_{R} \ldots \otimes_{R} X_{n}\right)=\left(X_{1} \otimes_{R} \ldots \otimes_{R} X_{k}\right) \widehat{\otimes}_{R}\left(X_{k+1} \otimes_{R} \ldots \otimes_{R} X_{n}\right),
$$

for $k \in\{1, \ldots, n-1\}$. It is generated by the cyclic tensor monomials $x_{1} \widehat{\otimes}_{R} \ldots \widehat{\otimes}_{R} x_{n}$. It is well known that the symmetry $c_{X, Y}: X \otimes_{\mathbb{K}} Y \rightarrow Y \otimes_{\mathbb{K}} X$ induces a natural isomorphism $i_{X, Y}$ : 
$X \widehat{\otimes}_{R} Y \cong Y \widehat{\otimes}_{R} X$. In particular, there is a natural isomorphism $i_{X_{1}, \ldots, X_{n}}: X_{1} \widehat{\otimes}_{R} \ldots \widehat{\otimes}_{R} X_{n} \rightarrow$ $X_{2} \widehat{\otimes}_{R} \ldots \widehat{\otimes}_{R} X_{n} \widehat{\otimes}_{R} X_{1}$, that maps a generator $x_{1} \widehat{\otimes}_{R} \ldots \widehat{\otimes}_{R} x_{n}$ to $x_{2} \widehat{\otimes}_{R} \ldots \widehat{\otimes}_{R} x_{n} \widehat{\otimes}_{R} x_{1}$.

As we have noticed in Example 1.5, the associativity constraint of the monoidal category $R$-Mod- $R$ defines a distributive law $\boldsymbol{t}: \boldsymbol{T}_{\boldsymbol{r}} \boldsymbol{T}_{\boldsymbol{l}} \rightarrow \boldsymbol{T}_{\boldsymbol{l}} \boldsymbol{T}_{\boldsymbol{r}}$. Thus, in this particular case that we are investigating, $\boldsymbol{t} X$ is the canonical isomorphism $\left(T \otimes_{R} X\right) \otimes_{R} T \cong T \otimes_{R}\left(X \otimes_{R} T\right)$, for any $X$ in $R$-Mod- $R$. Let us define $\boldsymbol{i} X: \boldsymbol{\Pi} \boldsymbol{T}_{\boldsymbol{l}} X \rightarrow \boldsymbol{\Pi} \boldsymbol{T}_{\boldsymbol{r}} X$ by $\boldsymbol{i} X:=\boldsymbol{i}_{T, X}$, as in Remark 1.14. In terms of these natural transformations we can give one of the main examples of admissible septuples.

Proposition 1.15. Let $\varphi: R \rightarrow T$ be a morphism of $\mathbb{K}$-algebras. The following data:

- the categories $\mathcal{M}:=R$-Mod- $R$ and $\mathcal{C}:=\operatorname{Mod}-\mathbb{K}$,

- the monads $\boldsymbol{T}_{\boldsymbol{l}}:=T \otimes_{R}(-)$ and $\boldsymbol{T}_{\boldsymbol{r}}:=(-) \otimes_{R} T$,

- the functor $\Pi: \mathcal{M} \rightarrow \mathcal{C}, \Pi X:=R \otimes_{R^{e}} X$,

- the natural transformation $\boldsymbol{t} X:\left(T \otimes_{R} X\right) \otimes_{R} T \rightarrow T \otimes_{R}\left(X \otimes_{R} T\right)$ defined by the canonical isomorphism;

- the natural transformation $i X: T \widehat{\otimes}_{R} X \rightarrow X \widehat{\otimes}_{R} T, t \widehat{\otimes}_{R} x \mapsto x \widehat{\otimes}_{R} t$,

define an admissible septuple $\mathcal{S}_{T}$.

Proof. Let $X$ be an $R$-bimodule. By definition,

$$
\boldsymbol{\Pi} \boldsymbol{T}_{l}^{n} X=R \otimes_{R^{e}}\left(T^{\otimes_{R} n} \otimes_{R} X\right) \cong T^{\widehat{\otimes}_{R} n} \widehat{\otimes}_{R} X \quad \text { and } \quad \boldsymbol{\Pi} \boldsymbol{T}_{r}{ }^{n} X \cong X \widehat{\otimes}_{R} T^{\widehat{\otimes}_{R}{ }^{n}} .
$$

Via these identifications, $\boldsymbol{i} \boldsymbol{T}_{\boldsymbol{l}} X=\boldsymbol{i}_{T, T, X}$ and $\boldsymbol{i} \boldsymbol{T}_{\boldsymbol{r}} X=\boldsymbol{i}_{T, X, T}$. So the conditions (1.2) take the form

$$
\boldsymbol{i}_{T, X} \circ\left(\varphi \widehat{\otimes}_{R} X\right)=X \widehat{\otimes}_{R} \varphi \quad \text { and } \quad \boldsymbol{i}_{T, X} \circ\left(m_{T} \widehat{\otimes}_{R} X\right)=\left(X \widehat{\otimes}_{R} m_{T}\right) \circ \boldsymbol{i}_{T \otimes_{R} T, X},
$$

identities which are obvious.

Let $\mathcal{S}_{T}$ be the admissible septuple associated to an algebra morphism $\varphi: R \rightarrow T$. A morphism of $R$-bimodules $w: X \otimes_{R} T \rightarrow T \otimes_{R} X$ is a transposition map in $\mathcal{W}_{\mathcal{S}_{T}}$ if, and only if, it satisfies the conditions

$$
w \circ\left(X \otimes_{R} \varphi\right)=\varphi \otimes_{R} X \quad \text { and } \quad w \circ\left(X \otimes_{R} m_{T}\right)=\left(m_{T} \otimes_{R} X\right) \circ\left(T \otimes_{R} w\right) \circ\left(w \otimes_{R} T\right),
$$

where $m_{T}: T \otimes_{R} T \rightarrow T$ denotes the multiplication map $t \otimes_{R} t^{\prime} \mapsto t t^{\prime}$. Note in passing the similarity of conditions (1.12) to some of those defining an entwining structure over $R$. (For the definition of entwining structures see [BMa, Definition 2.1], and for a reformulation over an arbitrary base algebra $R$ see [BB, Section 2.3].) By Proposition 1.9, there is a bijective correspondence between transposition maps $w: X \otimes_{R} T \rightarrow T \otimes_{R} X$ on one hand, and right $T$-actions on $T \otimes_{R} X$, which are left $T$-module maps with respect to the left $T$-action $t^{\prime} \cdot\left(t \otimes_{R} x\right)=t^{\prime} t \otimes_{R} x$, on the other hand.

THEOREM 1.16. Let $\mathcal{S}_{T}$ be the admissible septuple associated to an algebra morphism $\varphi: R \rightarrow T$. Let $w: X \otimes_{R} T \rightarrow T \otimes_{R} X$ be a transposition map in $\mathcal{W}_{\mathcal{S}_{T}}$, that is, a morphism of $R$-bimodules satisfying (1.12). Then there is a cocylic quotient $\widehat{Z}^{*}\left(\mathcal{S}_{T}, w\right)$ of $T^{\widehat{\otimes}_{R}{ }^{*+1}} \widehat{\otimes}_{R} X$ such that its cocyclic structure is induced by the para-cocyclic morphisms $w_{n}: T^{\widehat{\otimes}_{R} n+1} \widehat{\otimes}_{R} X \rightarrow T^{\widehat{\otimes}_{R} n+1} \widehat{\otimes}_{R} X$,

$$
w_{n}:=\left(T^{\widehat{\otimes}_{R} n} \widehat{\otimes}_{R} w\right) \circ \boldsymbol{i}_{T, \ldots, T, X},
$$

where $\boldsymbol{i}_{T, \ldots, T, X}$ is the $\mathbb{K}$-linear map defined in Remark 1.14.

Proof. Apply Theorem 1.10 for $\mathcal{S}=\mathcal{S}_{T}$. It yields a para-cocyclic object $Z^{*}\left(\mathcal{S}_{T}, w\right)$ whose paracocyclic operator is given in formula (1.8). For $\mathcal{S}_{T}$, the map $\boldsymbol{t}_{n}$ is the identity morphism of $T^{\widehat{\otimes}_{R} n+1} \widehat{\otimes}_{R} X$, cf. (1.4). Hence $w_{n}$ satisfies (1.13). We conclude the proof by applying Corollary 1.11 .

Corollary 1.17. Let $\mathcal{S}_{T}$ be the admissible septuple associated to a $\mathbb{K}$-algebra homomorphism $\varphi: R \rightarrow T$ as in Proposition 1.15. Then the canonical isomorphism $w_{T}: R \otimes_{R} T \rightarrow T \otimes_{R} R$ is a 
transposition map in $\mathcal{W}_{\mathcal{S}_{T}}$. The corresponding cocyclic cosimplex $Z^{*}\left(\mathcal{S}_{T}, w_{T}\right)$ has in degree $n$ the $\mathbb{K}$-module $Z^{n}\left(\mathcal{S}_{T}, w_{T}\right)=T^{\widehat{\otimes}_{R} n+1}$. The coface and codegeneracy maps are

$$
\begin{aligned}
& d_{k}\left(t_{0} \widehat{\otimes}_{R} t_{1} \widehat{\otimes}_{R} \ldots \widehat{\otimes}_{R} t_{n-1}\right)=t_{0} \widehat{\otimes}_{R} t_{1} \widehat{\otimes}_{R} \ldots \widehat{\otimes}_{R} t_{k-1} \widehat{\otimes}_{R} 1_{T} \widehat{\otimes}_{R} t_{k} \widehat{\otimes}_{R} \ldots \widehat{\otimes}_{R} t_{n-1} \\
& s_{k}\left(t_{0} \widehat{\otimes}_{R} t_{1} \widehat{\otimes}_{R} \ldots \widehat{\otimes}_{R} t_{n+1}\right)=t_{0} \widehat{\otimes}_{R} t_{1} \widehat{\otimes}_{R} \ldots \widehat{\otimes}_{R} t_{k-1} \widehat{\otimes}_{R} t_{k} t_{k+1} \widehat{\otimes}_{R} t_{k+2} \widehat{\otimes}_{R} \ldots \widehat{\otimes}_{R} t_{n+1},
\end{aligned}
$$

where $k \in\{0, \ldots, n\}$. The cocyclic operator is given by

$$
w_{n}\left(t_{0} \widehat{\otimes}_{R} t_{1} \widehat{\otimes}_{R} \ldots \widehat{\otimes}_{R} t_{n}\right)=t_{1} \widehat{\otimes}_{R} t_{2} \widehat{\otimes}_{R} \ldots \widehat{\otimes}_{R} t_{n} \widehat{\otimes}_{R} t_{0}
$$

REMARK 1.18. As $u_{T}: k \rightarrow T$, the unit of a $\mathbb{K}$-algebra $T$, is an algebra map, we can apply Proposition 1.15 to get an admissible septuple $\mathcal{S}_{u_{T}}$. Corresponding transposition maps were also considered by Kaygun in [Kay06] to construct cocyclic $\mathbb{K}$-modules. His approach should be considered, however, dual to our one (see related remarks in the Introduction).

It follows by an observation in [Burr, page 11] that for the admissible septuple $\mathcal{S}_{T}$, associated to an algebra morphism $\varphi: R \rightarrow T$ in Proposition 1.15, any $R-T$ bimodule $Y$ admits a transposition morphism $w_{Y}: Y \otimes_{R} T \rightarrow T \otimes_{R} Y, y \otimes_{R} t \mapsto 1_{T} \otimes_{R} y \cdot t$. In particular, for any $R$-bimodule $X$, the pair $\left(X \otimes_{R} T,\left(u_{T} \otimes_{R} X \otimes_{R} T\right) \circ\left(X \otimes_{R} m_{T}\right)\right)$ is an object in $\mathcal{W}_{\mathcal{S}_{T}}$. Corresponding para-cocyclic objects are given in the following

EXAMPLE 1.19. Let $\mathcal{S}_{T}$ be the admissible septuple associated to a $\mathbb{K}$-algebra homomorphism $\varphi$ : $R \rightarrow T$ as in Proposition 1.15. For any $R-T$ bimodule $Y$, there is a para-cocyclic cosimplex $Z^{*}\left(\mathcal{S}_{T}, w_{Y}\right)$, given in degree $n$ by the $\mathbb{K}$-module $Z^{n}\left(\mathcal{S}_{T}, w_{Y}\right)=T^{\widehat{\otimes}_{R}{ }^{n+1} \widehat{\otimes}_{R} Y}$. The coface and codegeneracy maps are

$$
\begin{aligned}
& d_{k}\left(t_{0} \widehat{\otimes}_{R} t_{1} \widehat{\otimes}_{R} \ldots \widehat{\otimes}_{R} t_{n-1} \widehat{\otimes}_{R} y\right)=t_{0} \widehat{\otimes}_{R} t_{1} \widehat{\otimes}_{R} \ldots \widehat{\otimes}_{R} t_{k-1} \widehat{\otimes}_{R} 1_{T} \widehat{\otimes}_{R} t_{k} \widehat{\otimes}_{R} \ldots \widehat{\otimes}_{R} t_{n-1} \widehat{\otimes}_{R} y \\
& s_{k}\left(t_{0} \widehat{\otimes}_{R} t_{1} \widehat{\otimes}_{R} \ldots \widehat{\otimes}_{R} t_{n+1} \widehat{\otimes}_{R} y\right)=t_{0} \widehat{\otimes}_{R} t_{1} \widehat{\otimes}_{R} \ldots \widehat{\otimes}_{R} t_{k-1} \widehat{\otimes}_{R} t_{k} t_{k+1} \widehat{\otimes}_{R} t_{k+2} \widehat{\otimes}_{R} \ldots \widehat{\otimes}_{R} t_{n+1} \widehat{\otimes}_{R} y
\end{aligned}
$$

where $k \in\{0, \ldots, n\}$. The para-cocyclic operator is given by

$$
w_{n}\left(t_{0} \widehat{\otimes}_{R} t_{1} \widehat{\otimes}_{R} \ldots \widehat{\otimes}_{R} t_{n} \widehat{\otimes}_{R} y\right)=t_{1} \widehat{\otimes}_{R} \ldots \widehat{\otimes}_{R} t_{n} \widehat{\otimes}_{R} 1_{T} \widehat{\otimes}_{R} y \cdot t_{0} .
$$

Note that $w_{n}$ is degenerate in the sense that the cocyclic quotient of $Z^{*}\left(\mathcal{S}_{T}, w_{Y}\right)$ (cf. Corollary $1.11)$ is given by $\widehat{Z}^{n}\left(\mathcal{S}_{T}, w_{Y}\right)=R \widehat{\otimes}_{R} Y$, in every degree $n$.

Next we are going to associate an admissible septuple to every ribbon algebra. Recall that a ribbon algebra is an algebra $\left(T, m_{T}, u_{T}\right)$ in a braided monoidal category $\mathcal{M}$ together with an automorphism $\sigma: T \rightarrow T$ in $\mathcal{M}$ such that

$$
\sigma \circ u_{T}=u_{T} \quad \text { and } \quad \sigma \circ m_{T}=m_{T} \circ(\sigma \otimes \sigma) \circ \boldsymbol{c}_{T, T}^{2} .
$$

Ribbon algebras appeared in [AM], where they are used to define cyclic homology of quasialgebras (non-associative algebras that are obtained by a cochain twist). We shall show that the ribbon automorphism $\sigma$ can be used to define a certain admissible septuple. For, we start with an algebra $\left(T, m_{T}, u_{T}\right)$ and an automorphism $\sigma: T \rightarrow T$ in a braided monoidal category. It is easy to see that $T$ is also an associative and unital algebra with respect to $m_{T}^{\prime}:=m_{T} \circ \boldsymbol{c}_{T, T}$ and $u_{T}^{\prime}:=u_{T}$. To make distinction between $T$ and the new algebra, latter one will be denoted by $T^{\prime}$.

Consider the monads $\boldsymbol{T}_{l}^{\prime}$ and $\boldsymbol{T}_{\boldsymbol{l}}$ on $\mathcal{M}$, defined as in Example 1.2. In the following, $\boldsymbol{T}_{\boldsymbol{l}}^{\prime}$ will play the role of $\boldsymbol{T}_{\boldsymbol{r}}$ in the definition of an admissible septuple. We have seen in Example 1.6 that $\boldsymbol{c}_{T, T}: T \otimes T \rightarrow T \otimes T$ induces a distributive law $\boldsymbol{t}: \boldsymbol{T}_{\boldsymbol{l}}^{\prime} \boldsymbol{T}_{\boldsymbol{l}} \rightarrow \boldsymbol{T}_{\boldsymbol{l}} \boldsymbol{T}_{\boldsymbol{l}}^{\prime}$

$$
\boldsymbol{t} X:=\boldsymbol{a}_{T, T, X} \circ\left(\boldsymbol{c}_{T, T} \otimes X\right) \circ \boldsymbol{a}_{T, T, X}^{-1},
$$

where $X$ is an arbitrary object in $\mathcal{M}$. Furthermore, we take $\mathcal{C}=\mathcal{M}$ and $\boldsymbol{\Pi}=\operatorname{Id}_{\mathcal{M}}$. By definition, the natural transformation $i: T_{l} \rightarrow T_{l}^{\prime}$ is

$$
\boldsymbol{i} X:=(\sigma \otimes X) .
$$

It is not difficult to prove that the relations in (1.2) hold if, and only if, the identities in (1.14) are satisfied. Thus, we have the following 
Proposition 1.20. The algebra $\left(T, m_{T}, u_{T}\right)$ in a braided monoidal category $\mathcal{M}$ is a ribbon algebra with ribbon automorphism $\sigma$ if and only if $\mathcal{S}_{T, \sigma}:=\left(\mathcal{M}, \mathcal{M}, \boldsymbol{T}_{\boldsymbol{l}}, \boldsymbol{T}_{l}^{\prime}, \operatorname{Id}_{\mathcal{M}}, \boldsymbol{t}, \boldsymbol{i}\right)$, the septuple constructed above, is admissible.

Let $\left(T, m_{T}, u_{T}\right)$ be a ribbon algebra with ribbon automorphism $\sigma$. In view of Proposition 1.20, we can speak about transposition morphisms with respect to $\mathcal{S}_{T, \sigma}$. A morphism $w: T \otimes X \rightarrow T \otimes X$ in $\mathcal{M}$ is a transposition map in $\mathcal{W}_{\mathcal{S}_{T, \sigma}}$ if, and only if

$$
\begin{aligned}
& w \circ\left(u_{T} \otimes X\right)=u_{T} \otimes X, \\
& w \circ\left(m_{T} \otimes X\right) \circ\left(\boldsymbol{c}_{T, T} \otimes X\right)=\left(m_{T} \otimes X\right) \circ(T \otimes w) \circ\left(\boldsymbol{c}_{T, T} \otimes X\right) \circ(T \otimes w) .
\end{aligned}
$$

Note that, in the second equation, we omitted the associativity constraints, to make the formula as short as possible. In fact, in view of the Coherence Theorem, we can omit bracketing in any equality involving morphisms in an arbitrary monoidal category $\mathcal{M}$. (For MacLane's Coherence Theorem consult e.g. [MacL, Theorem 1, p. 162] or [Mj, pp. 420-421]).

Relations (1.15) and (1.16) already appeared in the definition of braided twistors, structures that are used to construct new associative and unitary multiplications on $T \otimes T$. For details the reader is referred to [LPvO].

As an application of Theorem 1.10 we get Proposition 1.21 below. Note that, for simplifying the formulae of coface, codegeneracy and para-cocyclic morphisms, we omitted the associativity and unit constraints.

Proposition 1.21. Let $\left(T, m_{T}, u_{T}, \sigma\right)$ be a ribbon algebra in a braided monoidal category $\mathcal{M}$. For every object $X$ in $\mathcal{M}$, the sequence $Z^{n}\left(\mathcal{S}_{T, \sigma}, X\right):=T^{\otimes n+1} \otimes X$ defines a cosimplicial object, with respect to the coface and codegeneracy morphisms

$$
d_{k}:=T^{\otimes k} \otimes u_{T} \otimes T^{\otimes n-k} \otimes X \quad \text { and } \quad s_{k}:=T^{\otimes k} \otimes m_{T} \otimes T^{\otimes n-k} \otimes X,
$$

where $k \in\{0, \ldots, n\}$. Moreover, if $w: T \otimes X \rightarrow T \otimes X$ is a transposition map in $\mathcal{W}_{\mathcal{S}_{T, \sigma}}$, then $Z^{*}\left(\mathcal{S}_{T, \sigma}, X\right)$ admits a para-cocyclic structure with respect to the operator

$$
w_{n}:=\left(T^{\otimes n} \otimes w\right) \circ\left(T^{\otimes n-1} \otimes \boldsymbol{c}_{T, T} \otimes X\right) \circ \cdots \circ\left(\boldsymbol{c}_{T, T} \otimes T^{\otimes n-1} \otimes X\right) \circ\left(\sigma \otimes T^{\otimes n} \otimes X\right) .
$$

We shall denote this para-cocyclic object by $Z^{*}\left(\mathcal{S}_{T, \sigma}, w\right)$.

REMARKS 1.22. (i) Every ribbon algebra $(T, \sigma)$ in a braided category $(\mathcal{M}, \otimes, \boldsymbol{a}, \boldsymbol{l}, \boldsymbol{r}, \boldsymbol{c}, \mathbf{1})$ can be seen as an algebra with ribbon element $\sigma^{-1}$ in the opposite braided category of $\mathcal{M}$. Recall that the opposite of the braided category $\mathcal{M}$ is $(\mathcal{M}, \otimes, \boldsymbol{a}, \boldsymbol{l}, \boldsymbol{r}, \widetilde{\boldsymbol{c}}, \mathbf{1})$, where

$$
\widetilde{c}_{X, Y}=c_{Y, X}^{-1} \text {. }
$$

(ii) To every (para)-cocyclic object with invertible para-cocyclic morphism, there corresponds a (para)-cyclic object, namely its cyclic dual. Roughly speaking, the cyclic dual is obtained by interchanging the coface and codegeneracy morphisms and inverting the para-cocyclic operator. The interested reader can find the definition of the cyclic dual in [KR05]. The cyclic dual of $Z^{*}\left(\mathcal{S}_{T, \sigma^{-1}}, \operatorname{Id}_{T}\right)$ is, modulo a sign in the formula of $w_{n}$, the cyclic object in [AM, Theorem 4]. Note that, via the identification $T \otimes \mathbf{1} \cong T$, the identity morphism $\operatorname{Id}_{T}$ can be regarded as a transposition map in $\mathcal{W}_{\mathcal{S}_{T, \sigma^{-1}}}$. Thus, for an arbitrary $w$ in $\mathcal{W}_{\mathcal{S}_{T, \sigma^{-1}}}$, the cyclic dual of $Z^{*}\left(\mathcal{S}_{T, \sigma^{-1}}, w\right)$ may be interpreted as a generalization of cyclic homology introduced in [AM].

Other examples of para-cocyclic objects, obtained as applications of Theorem 1.10, will be discussed in Theorem 2.4 and Theorem 2.7.

1.E. The dual construction. In this section we turn to studying the situation dual to that in Section 1.C, i.e. application of Theorem 1.10 to the opposite categories $\mathcal{C}^{o p}$ and $\mathcal{M}^{o p}$. By $\mathcal{M}^{o p}$ we mean the category with the same classes of objects and morphisms in $\mathcal{M}$, with composition opposite to that in $\mathcal{M}$. Note that any diagram expressing an identity of morphisms in $\mathcal{M}$, yields a diagram in $\mathcal{M}^{o p}$, by interchanging the top and the bottom. In particular, a comonad on $\mathcal{M}$ is a monad on $\mathcal{M}^{o p}$. That is, a triple $\left(\boldsymbol{T}_{\boldsymbol{l}}, \Delta_{\boldsymbol{T}_{l}}, \boldsymbol{\varepsilon}_{\boldsymbol{T}_{l}}\right)$, consisting of a functor $\boldsymbol{T}_{\boldsymbol{l}}: \mathcal{M} \rightarrow \mathcal{M}$ and natural 
transformations $\Delta_{\boldsymbol{T}_{l}}: \boldsymbol{T}_{l} \rightarrow \boldsymbol{T}_{l}^{2}$ and $\varepsilon_{\boldsymbol{T}_{l}}: \boldsymbol{T}_{l} \rightarrow \boldsymbol{I} \boldsymbol{d}_{\mathcal{M}}$. Their compatibility axioms are obtained by reversing the arrows in the first two diagrams in Figure 2. For two comonads $\left(\boldsymbol{T}_{l}, \Delta_{\boldsymbol{T}_{l}}, \varepsilon_{\boldsymbol{T}_{l}}\right)$ and $\left(\boldsymbol{T}_{\boldsymbol{r}}, \Delta_{\boldsymbol{T}_{r}}, \varepsilon_{\boldsymbol{T}_{r}}\right)$ on a category $\mathcal{M}$, a dual distributive law is a distributive law for the monads $\boldsymbol{T}_{l}$ and $\boldsymbol{T}_{\boldsymbol{r}}$ on $\mathcal{M}^{o p}$. That is, a natural transformation $\boldsymbol{t}: \boldsymbol{T}_{l} \boldsymbol{T}_{\boldsymbol{r}} \rightarrow \boldsymbol{T}_{\boldsymbol{r}} \boldsymbol{T}_{l}$ such that the relations encoded in the up-down mirror images of the diagrams in Figure 3 hold.

To dualize admissible septuples we need two comonads $\boldsymbol{T}_{\boldsymbol{l}}$ and $\boldsymbol{T}_{\boldsymbol{r}}$ on a category $\mathcal{M}$, a dual distributive law $\boldsymbol{t}: \boldsymbol{T}_{l} \boldsymbol{T}_{\boldsymbol{r}} \rightarrow \boldsymbol{T}_{r} \boldsymbol{T}_{l}$, a covariant functor $\boldsymbol{\Pi}: \mathcal{M} \rightarrow \mathcal{C}$ and a natural transformation $i: \Pi T_{r} \rightarrow \Pi T_{l}$ that satisfy the identities

$$
\Pi \varepsilon_{T_{l}} \circ i=\Pi \varepsilon_{T_{r}} \quad \text { and } \quad \Pi \Delta_{T_{l}} \circ i=i T_{l} \circ \Pi t \circ i T_{r} \circ \Pi \Delta_{T_{r}} .
$$

Such a dual admissible septuple $\mathcal{S}^{0}=\left(\mathcal{M}, \mathcal{C}, \boldsymbol{T}_{\boldsymbol{l}}, \boldsymbol{T}_{\boldsymbol{r}}, \boldsymbol{\Pi}, \boldsymbol{t}, \boldsymbol{i}\right)$ determines a simplicial object $Z_{*}\left(\mathcal{S}^{0}, X\right)$ in $\mathcal{C}$, which in degree $n$ is given by $Z_{n}\left(\mathcal{S}^{0}, X\right)=\boldsymbol{\Pi} \boldsymbol{T}_{l}{ }^{n+1} X$. Its face maps $d_{k}: \boldsymbol{\Pi} \boldsymbol{T}_{l}{ }^{n+1} X \rightarrow \boldsymbol{\Pi} \boldsymbol{T}_{l}{ }^{n} X$ and degeneracy maps $s_{k}: \boldsymbol{\Pi} \boldsymbol{T}_{l}^{n+1} X \rightarrow \boldsymbol{\Pi} \boldsymbol{T}_{l}^{n+2} X$ are

$$
d_{k}:=\boldsymbol{\Pi} \boldsymbol{T}_{l}^{k} \varepsilon_{\boldsymbol{T}_{l}} \boldsymbol{T}_{l}^{n-k} X, \quad s_{k}:=\boldsymbol{\Pi} \boldsymbol{T}_{l}^{k} \Delta_{\boldsymbol{T}_{l}} \boldsymbol{T}_{l}{ }^{n-k} X,
$$

for any $k \in\{0, \ldots, n\}$. An arrow $w: \boldsymbol{T}_{l} X \rightarrow \boldsymbol{T}_{r} X$ in $\mathcal{M}$ is a transposition morphism with respect to the dual admissible septuple $\mathcal{S}^{0}$ if, and only if

$$
\boldsymbol{\varepsilon}_{\boldsymbol{T}_{r}} X \circ w=\varepsilon_{\boldsymbol{T}_{l}} X \quad \text { and } \quad \boldsymbol{\Delta}_{\boldsymbol{T}_{r}} X \circ w=\boldsymbol{T}_{\boldsymbol{r}} w \circ \boldsymbol{t} X \circ \boldsymbol{T}_{\boldsymbol{l}} w \circ \boldsymbol{\Delta}_{\boldsymbol{T}_{l}} X .
$$

Morphisms between transpositions maps can be easily defined by duality. The category of transposition maps with respect to $\mathcal{S}^{0}$ will be denoted by $\mathcal{W}_{\mathcal{S}^{0}}$.

Note that $\boldsymbol{t}$ can be lifted to a natural transformation $\boldsymbol{t}_{n}: \boldsymbol{\Pi} \boldsymbol{T}_{l}{ }^{n} \boldsymbol{T}_{\boldsymbol{r}} \rightarrow \boldsymbol{\Pi} \boldsymbol{T}_{\boldsymbol{r}} \boldsymbol{T}_{l}{ }^{n}$,

$$
\boldsymbol{t}_{n}^{0}:=\Pi \boldsymbol{t} \boldsymbol{T}_{l}^{n-1} \circ \boldsymbol{\Pi} \boldsymbol{T}_{l} \boldsymbol{t} \boldsymbol{T}_{l}^{n-2} \circ \ldots \circ \boldsymbol{\Pi \boldsymbol { T } _ { l }}{ }^{n-2} \boldsymbol{t} \boldsymbol{T}_{l} \circ \Pi \boldsymbol{T}_{l}{ }^{n-1} \boldsymbol{t} .
$$

Now we can state, for future references, the dual of Theorem 1.10.

Theorem 1.23. Consider a dual admissible septuple $\mathcal{S}^{0}$ as above and a transposition morphism $w: \boldsymbol{T}_{l} X \rightarrow \boldsymbol{T}_{\boldsymbol{r}} X$ in $\mathcal{W}_{\mathcal{S}^{0}}$. The simplex $Z_{*}\left(\mathcal{S}^{0}, X\right)$ is para-cyclic with para-cyclic morphism

$$
w_{n}:=\boldsymbol{i} \boldsymbol{T}_{l}^{n} X \circ \boldsymbol{t}_{n}^{0} X \circ \boldsymbol{\Pi} \boldsymbol{T}_{l}^{n} w .
$$

We shall denote this cyclic object by $Z_{*}\left(\mathcal{S}^{0}, w\right)$. For a morphism $f:(X, w) \rightarrow\left(X^{\prime}, w^{\prime}\right)$ in $\mathcal{W}_{\mathcal{S}^{0}}$, the morphisms $\boldsymbol{\Pi} \boldsymbol{T}_{l}{ }^{n+1} f: Z_{n}\left(\mathcal{S}^{0}, w\right) \rightarrow Z_{n}\left(\mathcal{S}^{0}, w^{\prime}\right)$ determine a morphism of para-cyclic objects.

Dually to Example 1.12, we have the following

EXAmple 1.24. Let $\boldsymbol{T}=(\boldsymbol{T}, \boldsymbol{\Delta}, \boldsymbol{\varepsilon})$ be a comonad on a category $\mathcal{M}$ and $\boldsymbol{t}: \boldsymbol{T} \boldsymbol{T} \rightarrow \boldsymbol{T} \boldsymbol{T}$ a dual distributive law. Assume that $\boldsymbol{t}$ satisfies the Yang-Baxter relation 1.11 and $\boldsymbol{t} \circ \boldsymbol{t} \circ \boldsymbol{\Delta}=\boldsymbol{\Delta}$. Then $\boldsymbol{T}^{0}=(\boldsymbol{T}, \boldsymbol{t} \circ \Delta, \varepsilon)$ is a comonad, and $\boldsymbol{t}$ can be regarded as a distributive law $\boldsymbol{T}^{\mathbf{0}} \boldsymbol{T} \rightarrow \boldsymbol{T} \boldsymbol{T}^{\mathbf{0}}$. Furthermore, the datum $\mathcal{S}:=\left(\mathcal{M}, \mathcal{M}, \boldsymbol{T}^{0}, \boldsymbol{T}, \operatorname{Id}_{\mathcal{M}}, \boldsymbol{t}, \operatorname{Id}_{\boldsymbol{T}}\right)$ is a dual admissible septuple. For any object $X$ in $\mathcal{M}$, the identity morphism $\operatorname{Id}_{\boldsymbol{T} X}$ is a transposition morphism. The corresponding para-cyclic morphism is $\boldsymbol{t}_{\boldsymbol{n}}^{\mathbf{0}}$ in (1.19). Note that if in addition $\boldsymbol{t}$ is an invertible morphism in $\mathcal{M}$ then its properties assumed above are equivalent to the premises in [Šk, Theorem 1].

Let $R$ be an algebra over a commutative ring $\mathbb{K}$. It was recalled in Example 1.2 that $R$-Mod- $R$, the category of $R$-bimodules, is monoidal with respect to the tensor product $\otimes_{R}$ and unit object $R$. By definition, an $R$-coring $(C, \Delta, \epsilon)$ is a coalgebra in $\left(R\right.$-Mod- $\left.R, \otimes_{R}, R\right)$.

Proposition 1.25. Let $\left(C, \Delta_{C}, \epsilon_{C}\right)$ be an $R$-coring. The following data:

- the category $\mathcal{C}:=\operatorname{Mod}-\mathbb{K}$ of $\mathbb{K}$-modules and the category $\mathcal{M}:=R$-Mod- $R$ of $R$-bimodules,

- the comonads $\boldsymbol{T}_{l}:=C \otimes_{R}(-)$ and $\boldsymbol{T}_{\boldsymbol{r}}:=(-) \otimes_{R} C$ on $R$-Mod- $R$,

- the functor $\Pi: R$-Mod- $R \rightarrow \operatorname{Mod}-\mathbb{K}, M \mapsto R \otimes_{R^{e}} M$ in Definition 1.13,

- the trivial dual distributive law $\boldsymbol{t} X: C \otimes_{R}\left(X \otimes_{R} C\right) \rightarrow\left(C \otimes_{R} X\right) \otimes_{R} C$,

- the natural morphism $i X: X \widehat{\otimes}_{R} C \rightarrow C \widehat{\otimes}_{R} X$, given by the flip map $x \widehat{\otimes}_{R} c \mapsto c \widehat{\otimes}_{R} x$,

define a dual admissible septuple $\mathcal{S}_{C}$. 
Proof. We have to check the identities in (1.17). Recall that, for any $R$-bimodule $X$, the cyclic tensor product $\Pi(X):=R \otimes_{R^{e}} X$ is isomorphic to the quotient of $X$ modulo the $\mathbb{K}$-submodule $[X, R]$ generated by all commutators $[x, r]$, where $x \in X$ and $r \in R$. Hence $\widehat{r x}=\widehat{x r}$, where $\widehat{z}$ denotes the class of $z$ in the quotient module, for any $z \in X$. To prove the first relation in (1.17), note that

$$
\left(\Pi \varepsilon_{T_{l}} \circ i\right)\left(x \widehat{\otimes}_{R} c\right)=\widehat{\varepsilon_{C}(c) x}=\widehat{x \varepsilon_{C}(c)}=\Pi \varepsilon_{T_{r}}\left(x \widehat{\otimes}_{R} c\right) .
$$

For the coproduct in the coring $C$ we use a Sweedler type notation, namely we write $\Delta_{C}(c)=$ $c_{(1)} \otimes_{R} c_{(2)}$, with implicit summation understood. A straightforward computation yields

$$
\left(\boldsymbol{\Pi} \boldsymbol{\Delta}_{\boldsymbol{T}_{l}} \circ \boldsymbol{i}\right)\left(x \widehat{\otimes}_{R} c\right)=c_{(1)} \widehat{\otimes}_{R} c_{(2)} \widehat{\otimes}_{R} x=\left(\boldsymbol{i} \boldsymbol{T}_{\boldsymbol{l}} \circ \boldsymbol{\Pi} \boldsymbol{t} \circ \boldsymbol{i} \boldsymbol{T}_{\boldsymbol{r}} \circ \boldsymbol{\Pi} \boldsymbol{\Delta}_{\boldsymbol{T}_{\boldsymbol{r}}}\right)\left(x \widehat{\otimes}_{R} c\right),
$$

for any $x \in X$ and $c \in C$. Thus the second relation in (1.17) is also proven.

Let $\mathcal{S}_{C}$ be the dual admissible septuple associated to an $R$-coring $\left(C, \Delta_{C}, \epsilon_{C}\right)$. In this particular case, a map of $R$-bimodules $w: C \otimes_{R} X \rightarrow X \otimes_{R} C$ is a transposition map in $\mathcal{W}_{\mathcal{S}_{C}}$ if, and only if,

$$
\left(X \otimes_{R} \varepsilon_{C}\right) \circ w=\varepsilon_{C} \otimes_{R} X \quad \text { and } \quad\left(X \otimes_{R} \Delta_{C}\right) \circ w=\left(w \otimes_{R} C\right) \circ\left(C \otimes_{R} w\right) \circ\left(\Delta_{C} \otimes_{R} X\right) .
$$

TheOREM 1.26. Let $\mathcal{S}_{C}$ be the dual admissible septuple associated to an $R$-coring, as in Proposition 1.25. Let $w: C \otimes_{R} X \rightarrow X \otimes_{R} C$ be a transposition map in $\mathcal{W}_{\mathcal{S}_{C}}$, that is, a morphism of $R$ bimodules satisfying (1.21). Then there is a cyclic subobject $\widehat{Z}_{*}\left(\mathcal{S}_{C}, w\right)$ of $C^{\widehat{\otimes}_{R} *+1} \widehat{\otimes}_{R} X$ whose cyclic structure is restriction of the para-cyclic morphism $w_{n}: C^{\widehat{\otimes}_{R}{ }^{n+1}} \widehat{\otimes}_{R} X \rightarrow C^{\widehat{\otimes}_{R}{ }^{n+1}} \widehat{\otimes}_{R} X$,

$$
w_{n}:=i_{C, \ldots, C, X}^{-1} \circ\left(C^{\widehat{\otimes}_{R} n} \widehat{\otimes}_{R} w\right),
$$

where $\boldsymbol{i}_{C, \ldots, C, X}$ is the isomorphism constructed in Remark 1.14.

Proof. Proceed as in the proof of Theorem 1.16.

Dually to Corollary 1.17, the following holds.

Corollary 1.27. Let $\mathcal{S}_{C}$ be the dual admissible septuple associated to an $R$-coring, as in Proposition 1.25. Then the canonical isomorphism $w_{C}: C \otimes_{R} R \rightarrow R \otimes_{R} C$ is a transposition map in $\mathcal{W}_{\mathcal{S}_{C}}$. The corresponding cyclic object $Z_{*}\left(\mathcal{S}_{C}, w_{C}\right)$ has in degree $n$ the $\mathbb{K}$-module $Z_{n}\left(\mathcal{S}_{C}, w_{C}\right)=C^{\widehat{\otimes}_{R} n+1}$. The face and degeneracy maps are

$$
\begin{array}{ll}
d_{k}\left(c_{0} \widehat{\otimes}_{R} c_{1} \widehat{\otimes}_{R} \ldots \widehat{\otimes}_{R} c_{n}\right)= \begin{cases}c_{0} \widehat{\otimes}_{R} \ldots \widehat{\otimes}_{R} \epsilon_{C}\left(c_{k}\right) c_{k+1} \widehat{\otimes}_{R} \ldots \widehat{\otimes}_{R} c_{n}, & \text { for } 0 \leq k<n, \\
c_{0} \widehat{\otimes}_{R} c_{1} \widehat{\otimes}_{R} \ldots \widehat{\otimes}_{R} c_{n-2} \widehat{\otimes}_{R} c_{n-1} \epsilon_{C}\left(c_{n}\right), & \text { for } k=n,\end{cases} \\
s_{k}\left(c_{0} \widehat{\otimes}_{R} c_{1} \widehat{\otimes}_{R} \ldots \widehat{\otimes}_{R} c_{n}\right)=c_{0} \widehat{\otimes}_{R} \ldots \widehat{\otimes}_{R} c_{k-1} \widehat{\otimes}_{R} \Delta_{C}\left(c_{k}\right) \widehat{\otimes}_{R} c_{k+1} \widehat{\otimes}_{R} \ldots \widehat{\otimes}_{R} c_{n}, & \text { for } 0 \leq k \leq n .
\end{array}
$$

The cyclic operator is defined by

$$
w_{n}\left(c_{0} \widehat{\otimes}_{R} \ldots \widehat{\otimes}_{R} c_{n}\right)=c_{n} \widehat{\otimes}_{R} c_{0} \widehat{\otimes}_{R} \ldots \widehat{\otimes}_{R} c_{n-1} .
$$

A symmetrical version of the construction in Corollary 1.27 is described in [Ra, Proposition 3.1].

EXAmple 1.28. Let $\varphi: S \rightarrow T$ be a homomorphism of algebras over a commutative ring $\mathbb{K}$. It determines the Sweedler's $T$-coring $T \otimes_{S} T$, where on $T \otimes_{S} T$ we take the obvious $T$-bimodule structure. The coproduct $\Delta_{T \otimes_{S} T}$ and the counit $\epsilon_{T \otimes_{S} T}$ are respectively defined by

$$
\begin{array}{lll}
\Delta_{T \otimes_{S} T}: & T \otimes_{S} T \rightarrow\left(T \otimes_{S} T\right) \otimes_{T}\left(T \otimes_{S} T\right), & \Delta_{T \otimes_{S} T}\left(t \otimes_{S} t^{\prime}\right)=t \otimes_{S} 1_{T} \otimes_{S} t^{\prime}, \\
\epsilon_{T \otimes_{S} T}: & T \otimes_{S} T \rightarrow T, & \epsilon_{T \otimes_{S} T}\left(t \otimes_{S} t^{\prime}\right)=t t^{\prime},
\end{array}
$$

where in the definition of $\Delta_{T \otimes_{R} T}$ we identified $\left(T \otimes_{S} T\right) \otimes_{T}\left(T \otimes_{S} T\right)$ and $T \otimes_{S} T \otimes_{S} T$.

For $S$-bimodules $T$ and $X$, let $v: T \otimes_{S} X \rightarrow X \otimes_{S} T$ be an $S$-bimodule map. For $t \in T$ and $x \in X$ we shall use the notation $v\left(t \otimes_{S} x\right)=x_{v} \otimes_{S} t_{v}$, where in the right hand side implicit summation is understood. 
COROLlary 1.29. Let $\varphi: S \rightarrow T$ be a homomorphism of algebras over a commutative ring $\mathbb{K}, X$ be an $S$-bimodule and $v: T \otimes_{S} X \rightarrow X \otimes_{S} T$ be an $S$-bimodule map satisfying

$$
v \circ\left(\varphi \otimes_{S} X\right)=X \otimes_{S} \varphi \quad \text { and } \quad v \circ\left(m_{T} \otimes_{S} X\right)=\left(X \otimes_{S} m_{T}\right) \circ\left(v \otimes_{S} T\right) \circ\left(T \otimes_{S} v\right) .
$$

There is a cyclic object $Z_{*}(T / S, v)$, with $Z_{n}(T / S, v)=T^{\hat{\otimes}_{S}{ }^{n+1}} \hat{\otimes}_{S} X$ whose face and degeneracy maps are

$$
\begin{aligned}
& d_{k}\left(t_{0} \widehat{\otimes}_{S} t_{1} \widehat{\otimes}_{S} \cdots \widehat{\otimes}_{S} t_{n} \widehat{\otimes}_{S} x\right)= \begin{cases}t_{0} \widehat{\otimes}_{S} t_{1} \widehat{\otimes}_{S} \cdots \widehat{\otimes}_{S} t_{k} t_{k+1} \widehat{\otimes}_{S} \cdots \widehat{\otimes}_{S} t_{n} \widehat{\otimes}_{S} x, & \text { for } 0 \leq k<n, \\
\left(t_{n}\right)_{v} t_{0} \widehat{\otimes}_{S} t_{1} \widehat{\otimes}_{S} \cdots \widehat{\otimes}_{S} t_{n-1} \widehat{\otimes}_{S}(x)_{v}, & \text { for } k=n,\end{cases} \\
& s_{k}\left(t_{0} \widehat{\otimes}_{S} t_{1} \widehat{\otimes}_{S} \cdots \widehat{\otimes}_{S} t_{n} \widehat{\otimes}_{S} x\right)=t_{0} \widehat{\otimes}_{S} t_{1} \widehat{\otimes}_{S} \cdots \widehat{\otimes}_{S} t_{k} \widehat{\otimes}_{S} 1_{T} \widehat{\otimes}_{S} \cdots \widehat{\otimes}_{S} t_{n} \widehat{\otimes}_{S} x, \quad \text { for } 0 \leq k \leq n \text {. }
\end{aligned}
$$

The para-cyclic map is given by

$$
v_{n}\left(t_{0} \widehat{\otimes}_{S} \ldots \widehat{\otimes}_{S} t_{n} \widehat{\otimes}_{S} x\right)=\left(t_{n}\right)_{v} \widehat{\otimes}_{S} t_{0} \widehat{\otimes}_{S} \ldots \widehat{\otimes}_{S} t_{n-1} \widehat{\otimes}_{S}(x)_{v} .
$$

Proof. In terms of the map $v$, we can equip $X \otimes_{S} T$ with a $T$-bimodule structure by

$$
t_{1}\left(x \otimes_{S} t\right) t_{2}=v\left(t_{1} \otimes_{S} x\right) t t_{2} .
$$

Moreover,

$$
v \otimes_{S} T: T \otimes_{S} X \otimes_{S} T \cong\left(T \otimes_{S} T\right) \otimes_{T}\left(X \otimes_{S} T\right) \rightarrow\left(X \otimes_{S} T\right) \otimes_{T}\left(T \otimes_{S} T\right) \cong X \otimes_{S} T \otimes_{S} T
$$

is a transposition map for the Sweedler's $T$-coring $T \otimes_{S} T$ and the $T$-bimodule $X \otimes_{S} T$, in the sense of (1.18). Consequently, we can apply Corollary 1.27 to Sweedler's coring in Example 1.28. One proves that the corresponding para-cyclic object has $Z_{*}(T / S, v)$ as underlying simplicial structure and $v_{*}$ as para-cyclic map.

Remarks 1.30. (i) Let us take $X=S$. The canonical isomorphism $v: T \otimes_{S} S \rightarrow S \otimes_{S} T$ satisfies the hypothesis of Corollary 1.29. The corresponding para-cyclic (in fact cyclic) object was used in [JS] to define relative cyclic homology. Moreover, this cyclic object and the cocyclic object $Z^{*}\left(\mathcal{S}_{T}, w_{T}\right)$ in Corollary 1.17 are (cyclic) dual to each other. In the particular case when $R=k$ and $\varphi=u_{T}$, we rediscover the cyclic object introduced by A. Connes in order to define the cyclic homology of an algebra, cf. [We, p. 330]. Thus $Z^{*}\left(\mathcal{S}_{T}, w_{T}\right)$ is the cyclic dual of Connes' cyclic object.

(ii) Note that the construction of $Z_{*}(T / S, v)$ can be performed for any algebra $T$ in a symmetric monoidal category $\mathcal{M}$, by replacing everywhere $\widehat{\otimes}_{S}$ with $\otimes$, the tensor product in $\mathcal{M}$. Therefore, para-cyclic objects in [Kay06] and [HKRS2] are examples of this type.

(iii) If $w: X \otimes_{S} T \rightarrow T \otimes_{S} X$ is an invertible $S$-bimodule map satisfying (1.12), then $v=w^{-1}$ satisfies the relations in Corollary 1.29. Conversely, in the case when the morphism $v$ in the above construction is invertible, then its inverse is a transposition map in the sense of (1.12). As a matter of fact, the corresponding para-cocyclic object in Theorem 1.16 is cyclic dual of the para-cyclic object in Corollary 1.29. This suggests a categorical approach to cyclic duality, details of which will be studied elsewhere.

Other examples of para-cyclic objects, obtained as applications of Theorem 1.23, will be discussed in Theorem 2.9 and Theorem 2.11.

\section{Cyclic (CO)homology of Bialgebroids}

In this section we apply the categorical framework, obtained in Section 1, to examples provided by (co)module algebras and (co)module corings of bialgebroids, and analyze the structure of the resulting para-(co)cyclic objects. 
2.A. (Co)module algebras of bialgebroids. In this section we consider admissible septuples $\mathcal{S}_{T}$, coming from a $\mathbb{K}$-algebra homomorphism $\varphi: R \rightarrow T$ as in Proposition 1.15. As we have seen in Proposition 1.15, $\mathcal{S}_{T}$ determines a cosimplex

$$
Z^{n}\left(\mathcal{S}_{T}, X\right)=T^{\widehat{\otimes}_{R} n+1} \widehat{\otimes}_{R} X
$$

for any $R$-bimodule $X$. Coface and codegeneracy maps are given by

$$
\widehat{d}_{k}=T^{\widehat{\otimes}_{R} k} \widehat{\otimes}_{R} \varphi \widehat{\otimes}_{R} T^{\widehat{\otimes}_{R} n-k} \widehat{\otimes}_{R} X \quad \text { and } \quad \widehat{s}_{k}=T^{\widehat{\otimes}_{R} k} \widehat{\otimes}_{R} m_{T} \widehat{\otimes}_{R} T^{\widehat{\otimes}_{R} n-k} \widehat{\otimes}_{R} X,
$$

where $k=0, \ldots, n$ and $m_{T}$ denotes the multiplication map $T \otimes_{R} T \rightarrow T$. Furthermore, by Theorem 1.16, the cosimplex $Z^{*}\left(\mathcal{S}_{T}, X\right)$ is para-cocyclic provided that there is a transposition map $w: X \otimes_{R} T \rightarrow T \otimes_{R} X$. Conditions (1.12) characterizing a transposition map are reminiscent of some of the axioms of an entwining structure (over an algebra $R$ ), cf. [BB, Section 2.3]. Main examples of entwining structures over non-commutative algebras arise from Doi-Koppinen data of bialgebroids (in the sense of [BCM]). In a similar manner, the aim of this section is to construct canonical transposition maps in the case when $T$ is a (co)module algebra of an $R$-bialgebroid $\mathcal{B}$ and $X$ is a $\mathcal{B}$-(co)module.

Bialgebroids can be thought of as a generalization of bialgebras to arbitrary, non-commutative base algebras. The first form of the structure that is known today as a left bialgebroid was introduced by Takeuchi in [Ta] under the name $\times_{R}$-bialgebra. Another definition and the name 'bialgebroid' was proposed by $\mathrm{Lu}$ in [Lu]. The two definitions were proven to be equivalent in [BMi]. 'Left' and 'right' versions of bialgebroids were defined in [KSz].

Definition 2.1. Consider an algebra $R$ over a commutative ring $\mathbb{K}$. A left bialgebroid $\mathcal{B}$ over $R$ consists of the data $(B, \xi, \zeta, \Delta, \epsilon)$. Here $B$ is a $\mathbb{K}$-algebra and $\xi$ and $\zeta$ are $\mathbb{K}$-algebra homomorphisms $R \rightarrow B$ and $R^{o p} \rightarrow B$, respectively, such that their ranges are commuting subalgebras in $B$. In terms of the maps $\xi$ and $\zeta, B$ can be equipped with an $R$-bimodule structure as

$$
r_{1} \cdot b \cdot r_{2}:=\xi\left(r_{1}\right) \zeta\left(r_{2}\right) b, \quad \text { for } r_{1}, r_{2} \in R \quad \text { and } b \in B .
$$

By definition, the coproduct $\Delta: B \rightarrow B \otimes_{R} B$ and the counit $\epsilon: B \rightarrow R$ equip this bimodule with an $R$-coring structure. Between the algebra and coring structures of $B$ the following compatibility axioms are required. For the coproduct we introduce the index notation $\Delta(b)=b_{(1)} \otimes_{R} b_{(2)}$, where implicit summation is understood.

(i) $b_{(1)} \zeta(r) \otimes_{R} b_{(2)}=b_{(1)} \otimes_{R} b_{(2)} \xi(r)$, for $r \in R$ and $b \in B$.

(ii) $\Delta\left(1_{B}\right)=1_{B} \otimes_{R} 1_{B}$ and $\Delta\left(b b^{\prime}\right)=b_{(1)} b_{(1)}^{\prime} \otimes_{R} b_{(2)} b_{(2)}^{\prime}$, for $b, b^{\prime} \in B$.

(iii) $\epsilon\left(1_{B}\right)=1_{R}$ and $\epsilon\left(b b^{\prime}\right)=\epsilon\left(b \xi\left(\epsilon\left(b^{\prime}\right)\right)\right)$, for $b, b^{\prime} \in B$.

Axiom (i) in Definition 2.1 needs to be imposed in order for the second condition in axiom (ii) to make sense. Axiom (iii) implies that also $\epsilon\left(b b^{\prime}\right)=\epsilon\left(b \zeta\left(\epsilon\left(b^{\prime}\right)\right)\right)$, for $b, b^{\prime} \in B$. It follows by the $R$-module map properties, unitality and multiplicativity of the coproduct $\Delta$ that

$$
\Delta\left(\xi\left(r_{1}\right) \zeta\left(r_{2}\right) b \xi\left(r_{3}\right) \zeta\left(r_{4}\right)\right)=\xi\left(r_{1}\right) b_{(1)} \xi\left(r_{3}\right) \otimes_{R} \zeta\left(r_{2}\right) b_{(2)} \zeta\left(r_{4}\right),
$$

for $r_{1}, r_{2}, r_{3}, r_{4} \in R$ and $b \in \mathcal{B}$. Since the coproduct is coassociative, the Sweedler-Heynemann index notation can be used. That is, for the iterated power of the coproduct we write $\left(\Delta \otimes_{R} \mathcal{B} \otimes_{R}\right.$ $\left.\cdots \otimes_{R} \mathcal{B}\right) \circ \cdots \circ\left(\Delta \otimes_{R} \mathcal{B}\right) \circ \Delta(b)=b_{(1)} \otimes_{R} \cdots \otimes_{R} b_{(n-1)} \otimes_{R} b_{(n)}$, for any positive integer $n$ and $b \in \mathcal{B}$.

Note that the axioms in Definition 2.1 are not invariant under changing the multiplication in $B$ to the opposite multiplication. Definition 2.1 has a symmetrical counterpart, known as a right bialgebroid. For the details we refer to [KSz].

Definition 2.1 is motivated by the following result of Schauenburg. Consider two algebras $R$ and $B$ over a commutative ring $\mathbb{K}$ and two $\mathbb{K}$-algebra homomorphisms $\xi: R \rightarrow B$ and $\zeta: R^{o p} \rightarrow B$, whose ranges are commuting subalgebras of $B$. Clearly, in this setting any (left or right) $B$ module can be equipped with an $R$ bimodule structure using the maps $\xi$ and $\zeta$. For example, for a left $B$-module $V$ with action $\triangleright: B \otimes_{\mathbb{K}} V \rightarrow V$, one can define an $R$-bimodule structure as

$$
r_{1} \otimes_{\mathbb{K}} v \otimes_{\mathbb{K}} r_{2} \mapsto \xi\left(r_{1}\right) \zeta\left(r_{2}\right) \triangleright v .
$$


With respect to the resulting $R$-actions, $B$-module maps are $R$-bimodule maps. That is, there exists a forgetful functor from the category of (left or right) $B$-modules to the category of $R$-bimodules.

Theorem 2.2. [Sch98, Theorem 5.1] Consider two algebras $R$ and $B$ over a commutative ring $\mathbb{K}$ and two $\mathbb{K}$-algebra homomorphisms $\xi: R \rightarrow B$ and $\zeta: R^{o p} \rightarrow B$, whose ranges are commuting subalgebras of $B$. There exists a right (resp. left) bialgebroid $(B, \xi, \zeta, \Delta, \epsilon)$ if and only if the forgetful functor from the category of right (resp. left) $B$-modules to the category of R-bimodules is strict monoidal. That is, $R$ is a right (resp. left) $B$-module and the $R$-module tensor product of two right (resp. left) B-modules is a right (resp. left) B-module.

Similarly to the case of a bialgebra, in Theorem 2.2 for a left $R$-bialgebroid $(B, \xi, \zeta, \Delta, \epsilon)$ the following $B$-actions are used on $R$, and on the $R$-module tensor product of two left $B$-modules $V$ and $W$, respectively.

$$
b \triangleright r:=\epsilon(b \xi(r)) \quad \text { and } \quad b \triangleright\left(v \otimes_{R} w\right):=b_{(1)} \triangleright v \otimes_{R} b_{(2)} \triangleright w,
$$

for $r \in R, v \otimes_{R} w \in V \otimes_{R} W$ and $b \in B$. It was proven in [Sch98, Theorem 5.1] that the diagonal action in the second equation in (2.4) is meaningful by axiom (i) in Definition 2.1.

In light of Theorem 2.2, one can speak about right (resp. left) module algebras of a right (resp. left) bialgebroid $\mathcal{B}$, i.e. about algebras in the monoidal category of right (resp. left) $\mathcal{B}$-modules.

Definition 2.3. Consider an algebra $R$ over a commutative ring $\mathbb{K}$ and a left $R$-bialgebroid $\mathcal{B}$. A left $\mathcal{B}$-module algebra is a $\mathbb{K}$-algebra and left $\mathcal{B}$-module $T$, with $\mathcal{B}$-action $\triangleright: \mathcal{B} \otimes_{\mathbb{K}} T \rightarrow T$, such that the multiplication in $T$ is $R$-balanced and

$$
b \triangleright 1_{T}=\xi(\epsilon(b)) \triangleright 1_{T} \quad \text { and } \quad b \triangleright\left(t t^{\prime}\right)=\left(b_{(1)} \triangleright t\right)\left(b_{(2)} \triangleright t^{\prime}\right),
$$

for $b \in B$ and $t, t^{\prime} \in T$.

For example, the constituent algebra in a left bialgebroid $\mathcal{B}$ is itself a (so called left regular) left $\mathcal{B}$-module algebra via the action given by the product in $\mathcal{B}$.

Note that for a left module algebra $T$ of a left $R$-bialgebroid $\mathcal{B}=(B, \xi, \zeta, \Delta, \epsilon)$, there is a canonical $\mathbb{K}$-algebra homomorphism $R \rightarrow T, r \mapsto \xi(r) \triangleright 1_{T}$. Hence there is a corresponding admissible septuple as in Proposition 1.15.

A left comodule of a left $R$-bialgebroid $\mathcal{B}=(B, \xi, \zeta, \Delta, \epsilon)$ is defined as a left comodule of the underlying $R$-coring $(\mathcal{B}, \Delta, \epsilon)$. That is, a left $R$-module $X$, together with a left $R$-module map $X \rightarrow \mathcal{B} \otimes_{R} X, x \mapsto x_{[-1]} \otimes_{R} x_{[0]}$ (where implicit summation is understood), satisfying coassociativity and counitality axioms. Note that a left $\mathcal{B}$-comodule $X$ can be equipped with an $R$-bimodule structure by introducing a right $R$-action

$$
x \cdot r:=\epsilon\left(x_{[-1]} \xi(r)\right) \cdot x_{[0]}, \quad \text { for } r \in R \quad \text { and } \quad x \in X .
$$

With respect to the resulting bimodule structure, $\mathcal{B}$-comodule maps are $R$-bimodule maps. In particular, the left $\mathcal{B}$-coaction on $X$ is an $R$-bimodule map in the sense that, for $r, r^{\prime} \in R$ and $x \in X$,

$$
\left(r \cdot x \cdot r^{\prime}\right)_{[-1]} \otimes_{R}\left(r \cdot x \cdot r^{\prime}\right)_{[0]}=\xi(r) x_{[-1]} \xi\left(r^{\prime}\right) \otimes_{R} x_{[0]} .
$$

Furthermore, for any $x \in X$ and $r \in R$,

$$
x_{[-1]} \otimes_{R} x_{[0]} \cdot r=x_{[-1]} \zeta(r) \otimes_{R} x_{[0]} .
$$

THEOREM 2.4. Let $R$ be an algebra over a commutative ring $\mathbb{K}$ and let $\mathcal{B}$ be a left bialgebroid over $R$. Consider a left $\mathcal{B}$-module algebra $T$ with $\mathcal{B}$-action $\triangleright$ and a left $\mathcal{B}$-comodule $X$ with coaction $x \mapsto x_{[-1]} \otimes_{R} x_{[0]}$ (where implicit summation is understood). Then a transposition map for the admissible septuple $\mathcal{S}_{T}$, associated via Proposition 1.15 to the $\mathbb{K}$-algebra map $R \rightarrow T, r \mapsto \xi(r) \triangleright 1_{T}$, is given by

$$
w: X \otimes_{R} T \rightarrow T \otimes_{R} X, \quad x \otimes_{R} t \mapsto x_{[-1]} \triangleright t \otimes_{R} x_{[0]} .
$$

Hence the cosimplex (2.1) admits a para-cocyclic structure

$$
w_{n}\left(t_{0} \widehat{\otimes}_{R} \ldots \widehat{\otimes}_{R} t_{n} \widehat{\otimes}_{R} x\right)=t_{1} \widehat{\otimes}_{R} \ldots \widehat{\otimes}_{R} t_{n} \widehat{\otimes}_{R} x_{[-1]} \triangleright t_{0} \widehat{\otimes}_{R} x_{[0]} .
$$


Proof. The map (2.9) is a well defined left $R$-module homomorphism by (2.7). Its right $R$-module map property follows by (2.8). Conditions (1.12) follow by definition (2.5) of a module algebra as it is shown below. Denote by $\varphi$ the algebra homomorphism $R \rightarrow T, r \mapsto \xi(r) \triangleright 1_{T}=\zeta(r) \triangleright 1_{T}$. Omitting canonical isomorphisms $R \otimes_{R} X \cong X \cong X \otimes_{R} R$, we have

$$
\begin{aligned}
& \left(w \circ\left(X \otimes_{R} \varphi\right)\right)(x)=x_{[-1]} \triangleright 1_{T} \otimes_{R} x_{[0]}=\zeta\left(\epsilon\left(x_{[-1]}\right)\right) \triangleright 1_{T} \otimes_{R} x_{[0]}=1_{T} \otimes_{R} \epsilon\left(x_{[-1]}\right) \cdot x_{[0]}= \\
& 1_{T} \otimes_{R} x=\left(\left(\varphi \otimes_{R} X\right)\right)(x), \\
& \left(\left(m_{T} \otimes_{R} X\right) \circ\left(T \otimes_{R} w\right) \circ\left(w \otimes_{R} T\right)\right)\left(x \otimes_{R} t \otimes_{R} t^{\prime}\right)=\left(x_{[-1]} \triangleright t\right)\left(x_{[0][-1]} \triangleright t^{\prime}\right) \otimes_{R} x_{[0][0]}= \\
& \quad\left(x_{[-1](1)} \triangleright t\right)\left(x_{[-1](2)} \triangleright t^{\prime}\right) \otimes_{R} x_{[0]}=x_{[-1]} \triangleright t t^{\prime} \otimes_{R} x_{[0]}=\left(w \circ\left(X \otimes_{R} m_{T}\right)\right)\left(x \otimes_{R} t \otimes_{R} t^{\prime}\right) .
\end{aligned}
$$

Analogously to (2.6), also a right comodule $V$ of a right $R$-bialgebroid $\mathcal{B}=(B, \xi, \zeta, \Delta, \epsilon)$ can be equipped with an $R$-bimodule structure by introducing a left $R$-action

$$
r \cdot v:=v^{[0]} \cdot \epsilon\left(\xi(r) v^{[1]}\right), \quad \text { for } r \in R \quad \text { and } \quad v \in V,
$$

where $v \mapsto v^{[0]} \otimes_{R} v^{[1]}$ denotes the right $\mathcal{B}$-coaction on $V$, with implicit summation understood. Hence there exists a forgetful functor from the category of right $\mathcal{B}$-comodules to the category of $R$-bimodules. With this observation in mind, the next theorem follows by a symmetrical form of [Sch98, Proposition 5.6].

TheOREM 2.5. Consider an algebra $R$ over a commutative ring $\mathbb{K}$ and a right $R$-bialgebroid $\mathcal{B}$. Then the forgetful functor from the category of right $\mathcal{B}$-comodules to the category of $R$-bimodules is strict monoidal. That is, $R$ is a right $\mathcal{B}$-comodule and the $R$-module tensor product of two right $\mathcal{B}$-comodules is a right $\mathcal{B}$-comodule.

Similarly to a bialgebra, in Theorem 2.5 for a right $R$-bialgebroid $\mathcal{B}=(B, \xi, \zeta, \Delta, \epsilon)$ the following $\mathcal{B}$-coactions on $R$, and on the $R$-module tensor product $V \otimes_{R} W$ of two right $\mathcal{B}$-comodules $V$ and $W$, are used.

$R \rightarrow R \otimes_{R} \mathcal{B} \cong \mathcal{B}, \quad r \mapsto \xi(r) \quad$ and $\quad V \otimes_{R} W \rightarrow V \otimes_{R} W \otimes_{R} \mathcal{B}, \quad v \otimes_{R} w \mapsto v^{[0]} \otimes_{R} w^{[0]} \otimes_{R} v^{[1]} w^{[1]}$.

The coaction on $V \otimes_{R} W$ is well defined by the right bialgebroid versions of properties (2.7) and (2.8), i.e. the identities

$$
\left(r \cdot v \cdot r^{\prime}\right)^{[0]} \otimes_{R}\left(r \cdot v \cdot r^{\prime}\right)^{[1]}=v^{[0]} \otimes_{R} \xi(r) v^{[1]} \xi\left(r^{\prime}\right) \quad \text { and } \quad r \cdot v^{[0]} \otimes_{R} v^{[1]}=v^{[0]} \otimes_{R} \zeta(r) v^{[1]},
$$

for $r, r^{\prime} \in R$ and $v \in V$. Symmetrically, the forgetful functors from the category of left comodules of a right $R$-bialgebroid, and from the categories of right or left comodules of a left $R$-bialgebroid, to the category of $R$-bimodules are strict (anti-)monoidal.

In light of Theorem 2.5, one can speak about right comodule algebras of a right bialgebroid $\mathcal{B}$, i.e. about algebras in the monoidal category of right $\mathcal{B}$-comodules.

Definition 2.6. Consider an algebra $R$ over a commutative ring $\mathbb{K}$ and a right $R$-bialgebroid $\mathcal{B}$. A right $\mathcal{B}$-comodule algebra is a $\mathbb{K}$-algebra and right $\mathcal{B}$-comodule $T$, with coaction $t \mapsto t^{[0]} \otimes_{R} t^{[1]}$, such that the multiplication in $T$ is $R$-balanced and, for $t, t^{\prime} \in T$,

$$
1_{T}^{[0]} \otimes_{R} 1_{T}^{[1]}=1_{T} \otimes_{R} 1_{T} \quad \text { and } \quad\left(t t^{\prime}\right)^{[0]} \otimes_{R}\left(t t^{\prime}\right)^{[1]}=t^{[0]} t^{[0]} \otimes_{R} t^{[1]} t^{\prime[1]} .
$$

For example, the constituent algebra in a right bialgebroid $\mathcal{B}$ is itself a (so called right regular) right $\mathcal{B}$-comodule algebra via the coaction given by the coproduct in $\mathcal{B}$.

Note that the second condition in (2.13) is meaningful since the multiplication in $T$ is $R$-balanced and the second condition in (2.12) holds. For a right comodule algebra $T$ of a right $R$-bialgebroid $\mathcal{B}$, there is a canonical $\mathbb{K}$-algebra homomorphism $R \rightarrow T, r \mapsto r \cdot 1_{T}=1_{T} \cdot r$, in terms of which $r \cdot t=\left(r \cdot 1_{T}\right) t$ and $t \cdot r=t\left(r \cdot 1_{T}\right)$. Hence there is a corresponding admissible septuple as in Proposition 1.15 . 
THEOREM 2.7. Let $R$ be an algebra over a commutative ring $\mathbb{K}$ and let $\mathcal{B}$ be a right bialgebroid over $R$. Consider a right $\mathcal{B}$-comodule algebra $T$, with coaction $t \mapsto t^{[0]} \otimes_{R} t^{[1]}$ (where implicit summation is understood) and a right $\mathcal{B}$-module $X$ with action $\triangleleft$. Then a transposition map for the admissible septuple $\mathcal{S}_{T}$, associated via Proposition 1.15 to the $\mathbb{K}$-algebra map $R \rightarrow T, r \mapsto r \cdot 1_{T}=1_{T} \cdot r$, is given by

$$
w: X \otimes_{R} T \rightarrow T \otimes_{R} X, \quad x \otimes_{R} t \mapsto t^{[0]} \otimes_{R} x \triangleleft t^{[1]} .
$$

Hence the cosimplex (2.1) admits a para-cocyclic structure

$$
w_{n}\left(t_{0} \widehat{\otimes}_{R} \ldots \widehat{\otimes}_{R} t_{n} \widehat{\otimes}_{R} x\right)=t_{1} \widehat{\otimes}_{R} \ldots \widehat{\otimes}_{R} t_{n} \widehat{\otimes}_{R} t_{0}^{[0]} \widehat{\otimes}_{R} x \triangleleft t_{0}^{[1]} .
$$

Proof. The map $w$ is a well defined $R$-bimodule homomorphism by (2.12). Conditions (1.12) follow by definition (2.13) of a comodule algebra.

2.B. (Co)module corings of bialgebroids. In this section we consider comonads $\boldsymbol{T}_{\boldsymbol{l}}:=C \otimes_{R}(-)$ on the category of $R$-bimodules as in Proposition 1.25, determined by a coring $(C, \Delta, \epsilon)$ over an algebra $R$. Let $\Pi$ be the functor in Definition 1.13. By Proposition 1.25, for any $R$-bimodule $X$, there is an associated simplicial module $Z_{*}\left(\mathcal{S}_{C}, X\right)$, which in degree $n$ is given by

$$
Z_{n}\left(\mathcal{S}_{C}, X\right)=C^{\widehat{\otimes}_{R} n+1} \widehat{\otimes}_{R} X
$$

Face and degeneracy maps in degree $n$ are

$$
\widehat{d}_{k}=C^{\widehat{\otimes}_{R} k} \widehat{\otimes}_{R} \epsilon_{C} \widehat{\otimes}_{R} C^{\widehat{\otimes}_{R} n-k} \widehat{\otimes}_{R} X \text { and } \widehat{s}_{k}=C^{\widehat{\otimes}_{R} k} \widehat{\otimes}_{R} \Delta_{C} \widehat{\otimes}_{R} C^{\widehat{\otimes}_{R} n-k} \widehat{\otimes}_{R} X,
$$

for $k \in\{0, \ldots, n\}$. As we have seen in Proposition 1.25, we can choose a second comonad $\boldsymbol{T}_{\boldsymbol{r}}:=$ $(-) \otimes_{R} C$ on the category of $R$-bimodules, and a natural transformation $i:(-) \widehat{\otimes}_{R} C \rightarrow C \widehat{\otimes}_{R}(-)$, given by the flip map. Taking the trivial natural transformation $t: C \otimes_{R}(-) \otimes_{R} C \rightarrow C \otimes_{R}(-) \otimes_{R} C$, the conditions in (1.17) hold. By Theorem 1.26, the simplex $Z_{*}\left(\mathcal{S}_{C}, X\right)$ is para-cyclic provided that there exists a morphism $w: C \otimes_{R} X \rightarrow X \otimes_{R} C$, satisfying (1.21). Note the similarity of conditions (1.21) to some of the axioms of an entwining structure over $R$. Similarly to the way Doi-Koppinen data determine entwining structures, in this section we construct dual transposition maps in the case when $C$ is a (co)module coring of a bialgebroid $\mathcal{B}$ and $X$ is a $\mathcal{B}$-(co)module.

In light of (a symmetrical version of) Theorem 2.5, one can speak about left comodule corings of a left bialgebroid $\mathcal{B}$, i.e. about coalgebras in the monoidal category of left $\mathcal{B}$-comodules.

Definition 2.8. Consider an algebra $R$ over a commutative ring $\mathbb{K}$ and a left $R$-bialgebroid $\mathcal{B}=(B, \xi, \zeta, \Delta, \epsilon)$. A left $\mathcal{B}$-comodule coring is an $R$-coring and left $\mathcal{B}$-comodule $C$, with one and the same underlying $R$-bimodule structure, such that for $c \in C$

$$
\xi\left(\epsilon_{C}(c)\right)=\zeta\left(\epsilon_{C}\left(c_{[0]}\right)\right) c_{[-1]} \quad \text { and } \quad c_{[-1]}{\underset{R}{R}}_{[0](1)} \underset{R}{\otimes} c_{[0](2)}=c_{(1)[-1]} c_{(2)[-1]} \underset{R}{\otimes} c_{(1)[0]}{\underset{R}{R}}_{(2)[0]} c_{(2)}
$$

where $\epsilon_{C}$ is the counit and $\Delta_{C}: c \mapsto c_{(1)} \otimes_{R} c_{(2)}$ is the coproduct of $C$ and $c \mapsto c_{[-1]} \otimes_{R} c_{[0]}$ is the $\mathcal{B}$-coaction on $C$.

The second condition in (2.16) is meaningful by (2.7) and (2.8). For example, the constituent $R$-coring in a left $R$-bialgebroid $\mathcal{B}$ is itself a (so called left regular) left $\mathcal{B}$-comodule coring via the coaction given by the coproduct.

THEOREM 2.9. Let $R$ be an algebra over a commutative ring $\mathbb{K}$ and let $\mathcal{B}$ be a left bialgebroid over $R$. Consider a left $\mathcal{B}$-comodule coring $C$ with coaction $c \mapsto c_{[-1]} \otimes_{R} c_{[0]}$, and a left $\mathcal{B}$-module $X$ with action $\triangleright$. Then a transposition map $w: C \otimes_{R} X \rightarrow X \otimes_{R} C$ for the dual admissible septuple $\mathcal{S}_{C}$, associated via Proposition 1.25 to the $R$-coring $C$, is given by $w\left(c \otimes_{R} x\right):=c_{[-1]} \triangleright x \otimes_{R} c_{[0]}$. Hence the simplex (2.14) admits a para-cyclic structure

$$
w_{n}\left(c_{0} \widehat{\otimes}_{R} \ldots \widehat{\otimes}_{R} c_{n} \widehat{\otimes}_{R} x\right)=c_{n[0]} \widehat{\otimes}_{R} c_{0} \widehat{\otimes}_{R} \ldots \widehat{\otimes}_{R} c_{n-1} \widehat{\otimes}_{R} c_{n[-1]} \triangleright x .
$$

Proof. The map $w$ is a well defined $R$-bimodule homomorphism by (2.7) and (2.8). Conditions (1.21) follow by definition (2.16) of a comodule coring.

In light of Theorem 2.2, one can speak about right (resp. left) module corings of a right (resp. left) bialgebroid $\mathcal{B}$, i.e. about coalgebras in the monoidal category of right (resp. left) $\mathcal{B}$-modules. 
Definition 2.10. Consider an algebra $R$ over a commutative ring $\mathbb{K}$ and a right $R$-bialgebroid $\mathcal{B}=(B, \xi, \zeta, \Delta, \epsilon)$. A right $\mathcal{B}$-module coring is an $R$-coring and right $\mathcal{B}$-module $C$, with one and the same underlying $R$-bimodule structure, such that for $c \in C$ and $b \in B$

$$
\epsilon_{C}(c \triangleleft b)=\epsilon\left(\xi\left(\epsilon_{C}(c)\right) b\right) \quad \text { and } \quad(c \triangleleft b)^{(1)} \otimes_{R}(c \triangleleft b)^{(2)}=c^{(1)} \triangleleft b^{(1)} \otimes_{R} c^{(2)} \triangleleft b^{(2)},
$$

where $\epsilon_{C}$ is the counit and $\Delta_{C}: c \mapsto c^{(1)} \otimes_{R} c^{(2)}$ is the coproduct in $C$, the symbol $\triangleleft$ denotes the $\mathcal{B}$-action on $C$ and for the coproduct in $\mathcal{B}$ the index notation $\Delta: b \mapsto b^{(1)} \otimes_{R} b^{(2)}$ is used, with implicit summation understood.

For example, the constituent $R$-coring in a right $R$-bialgebroid $\mathcal{B}$ is itself a (so called right regular) right $\mathcal{B}$-module coring via the action given by the product in $\mathcal{B}$.

THEOREM 2.11. Let $R$ be an algebra over a commutative ring $\mathbb{K}$ and let $\mathcal{B}$ be a right bialgebroid over $R$. Consider a right $\mathcal{B}$-module coring $C$ with action $\triangleleft$, and a right $\mathcal{B}$-comodule $X$ with coaction $x \mapsto x^{[0]} \otimes_{R} x^{[1]}$. Then a transposition map $w: C \otimes_{R} X \rightarrow X \otimes_{R} C$, for the dual admissible septuple $\mathcal{S}_{C}$, associated via Proposition 1.25 to the $R$-coring $C$, is given by $w\left(c \otimes_{R} x\right):=x^{[0]} \otimes_{R} c \triangleleft x^{[1]}$. Hence the simplex (2.14) admits a para-cyclic structure

$$
w_{n}\left(c_{0} \widehat{\otimes}_{R} \ldots \widehat{\otimes}_{R} c_{n} \widehat{\otimes}_{R} x\right)=c_{n} \triangleleft x^{[1]} \widehat{\otimes}_{R} c_{0} \widehat{\otimes}_{R} \ldots \widehat{\otimes}_{R} c_{n-1} \widehat{\otimes}_{R} x^{[0]} .
$$

Proof. The map $w$ is a well defined $R$-bimodule homomorphism by (2.12). Conditions (1.21) follow by definition (2.17) of a module coring.

2.C. Stable anti Yetter-Drinfel'd modules of $\times_{R}$-Hopf algebras. For a right module coring $C$ and right comodule $X$ of a right bialgebroid $\mathcal{B}=(B, \xi, \zeta, \Delta, \epsilon)$ over a $\mathbb{K}$-algebra $R$, there is a $\mathbb{K}$-module isomorphism

$$
C^{\widehat{\otimes}_{R} n+1} \widehat{\otimes}_{R} X \cong C^{\otimes_{R} n+1} \otimes_{R^{e}} X .
$$

Assume that $X$ has an additional left $\mathcal{B}$-module structure. In this case, corresponding to the $\mathbb{K}$ algebra homomorphism $R^{e} \rightarrow B, r \otimes_{\mathbb{K}} r^{\prime} \mapsto \xi(r) \zeta\left(r^{\prime}\right)=\zeta\left(r^{\prime}\right) \xi(r)$, there is a canonical epimorphism $C^{\widehat{\otimes}_{R} n+1} \widehat{\otimes}_{R} X \rightarrow C^{\otimes_{R} n+1} \otimes_{\mathcal{B}} X$, where $C^{\otimes_{R} n+1}$ is understood to be a right $\mathcal{B}$-module via the diagonal action $\left(c_{1} \otimes_{R} \ldots \otimes_{R} c_{n+1}\right) \triangleleft b:=c_{1} \triangleleft b^{(1)} \otimes_{R} \ldots \otimes_{R} c_{n+1} \triangleleft b^{(n+1)}$, given by the iterated coproduct in $\mathcal{B}$. It is a well defined action by (a symmetrical version of) [Sch98, Theorem 5.1].

Lemma 2.12. Let $R$ be an algebra over a commutative ring $\mathbb{K}$ and let $\mathcal{B}$ be a right bialgebroid over $R$. Consider a right $\mathcal{B}$-module coring $C$ and a left $\mathcal{B}$-module right $\mathcal{B}$-comodule $X$. Then the simplex in Theorem 2.11 projects to a simplex $C^{\otimes_{R} n+1} \otimes_{\mathcal{B}} X$.

Proof. Since the coproduct $\Delta_{C}$ and the counit $\epsilon_{C}$ of $C$ are right $\mathcal{B}$-module maps by definition, face and degeneracy maps of the simplex in Example 1.27 are right $\mathcal{B}$-module maps with respect to the diagonal action. Hence we can take their tensor product with the identity map on $X$ over the algebra $\mathcal{B}$, yielding a simplex as stated.

The task of this section is to find criteria for the cyclicity of the quotient simplex in Lemma 2.12. In order to do so, some restriction on the involved bialgebroid is needed.

Definition 2.13. [Sch00, Theorem and Definition 3.5] Let $R$ be an algebra over a commutative ring $\mathbb{K}$. A right $R$-bialgebroid $\mathcal{B}=(B, \xi, \zeta, \Delta, \epsilon)$ is said to be a right $\times_{R}$-Hopf algebra provided that the map

$$
\vartheta: \mathcal{B} \otimes_{R^{o p}} \mathcal{B} \rightarrow \mathcal{B} \otimes_{R} \mathcal{B}, \quad b \otimes_{R^{o p}} b^{\prime} \mapsto b b^{\prime(1)} \otimes_{R} b^{\prime(2)}
$$

is bijective. In the domain of the map in (2.19), $R^{o p}$-module structures are given by right and left multiplication by $\zeta(r)$, for $r \in R$. In the codomain of the map in (2.19), $R$-module structures are given by right multiplication by $\xi(r)$ and $\zeta(r)$, for $r \in R$.

The notion of a $\times_{R}$-Hopf algebra extends that of a Hopf algebra. Indeed, if $\mathcal{B}$ is a bialgebra over a commutative ring $R$, with coproduct $b \mapsto b^{(1)} \otimes_{R} b^{(2)}$, then the map (2.19) is bijective if and only if $\mathcal{B}$ is a Hopf algebra. In this case the inverse is given in terms of the antipode $S$ as $\vartheta^{-1}\left(b \otimes_{R} b^{\prime}\right):=b S\left(b^{\prime(1)}\right) \otimes_{R} b^{\prime(2)}$. 
For an algebra $R$, consider a right $\times_{R}$-Hopf algebra $\mathcal{B}$. Since the map $\vartheta$ in $(2.19)$ is a left $\mathcal{B}$-module map, its inverse is determined by the restriction $\vartheta^{-1}\left(1_{\mathcal{B}} \otimes_{R} b\right)=: b_{-} \otimes_{R^{o p}} b_{+}$, where implicit summation is understood. Lemma 2.14, which is essentially a symmetrical version of [Sch00, Proposition 3.7], collects properties of the map $b \mapsto b_{-} \otimes_{R^{o p}} b_{+}$.

Lemma 2.14. For an algebra $R$, consider a right $\times_{R}$-Hopf algebra $\mathcal{B}=(B, \xi, \zeta, \Delta, \epsilon)$. Write $\Delta(b)=: b^{(1)} \otimes_{R} b^{(2)}$ for the coproduct and in terms of the map (2.19) put $b_{-} \otimes_{R^{o p}} b_{+}:=\vartheta^{-1}\left(1_{\mathcal{B}} \otimes_{R} b\right)$. The following identities hold, for $b, b^{\prime} \in \mathcal{B}$ and $r \in R$.

(i) $b_{-} b_{+}{ }^{(1)} \otimes_{R} b_{+}{ }^{(2)}=1_{\mathcal{B}} \otimes_{R} b$

(ii) $b^{(1)} b^{(2)}-\otimes_{R^{o p}} b^{(2)}+=1_{\mathcal{B}} \otimes_{R^{o p}} b$

(iii) $\left(b b^{\prime}\right)_{-} \otimes_{R^{o p}}\left(b b^{\prime}\right)_{+}=b_{-}^{\prime} b_{-} \otimes_{R^{o p}} b_{+} b_{+}^{\prime}$

(iv) $1_{\mathcal{B}-} \otimes_{R^{\text {op }}} 1_{\mathcal{B}+}=1_{\mathcal{B}} \otimes_{R^{o p}} 1_{\mathcal{B}}$

(v) $b_{-} \otimes_{R^{o p}} b_{+}{ }^{(1)} \otimes_{R} b_{+}{ }^{(2)}=b^{(1)}{ }_{-} \otimes_{R^{o p}} b^{(1)}+\otimes_{R} b^{(2)}$

(vi) $b_{-}{ }^{(1)} \otimes_{R} b_{-}{ }^{(2)} \otimes_{R^{o p}} b_{+}=b_{+-} \otimes_{R} b_{-} \otimes_{R^{o p}} b_{++}$

(vii) $b=\zeta\left(\epsilon\left(b_{-}\right)\right) b_{+}$

(viii) $b_{-} b_{+}=\xi(\epsilon(b))$

(ix) $\zeta(r) b_{-} \otimes_{R^{o p}} b_{+}=b_{-} \otimes_{R^{o p}} b_{+} \zeta(r)$.

Next Definition 2.15 extends [JŞ, Definition 4.1] or [HKRS1, Definition 2.1].

Definition 2.15. For an algebra $R$, consider a right $\times_{R}$ Hopf algebra $\mathcal{B}=(B, \xi, \zeta, \Delta, \epsilon)$. Let $X$ be a right $\mathcal{B}$-comodule and left $\mathcal{B}$-module. Denote the right $\mathcal{B}$-coaction on $X$ by $x \mapsto x^{[0]} \otimes_{R} x^{[1]}$, for $x \in X$ (where implicit summation is understood) and denote the left $\mathcal{B}$-action by $b \triangleright x$, for $b \in \mathcal{B}$ and $x \in X$. We say that $X$ is an anti Yetter-Drinfel'd module provided that the following conditions hold.

(i) The $R$-bimodule structures of $X$, underlying its module and comodule structures, coincide. That is, for $x \in X$ and $r \in R$,

$$
x \cdot r=\zeta(r) \triangleright x \quad \text { and } \quad r \cdot x=\xi(r) \triangleright x,
$$

where $x \cdot r$ denotes the right $R$-action on the right $\mathcal{B}$-comodule $X$ and $r \cdot x$ is the canonical left $R$-action (2.11) coming from the right $\mathcal{B}$-coaction.

(ii) For $b \in \mathcal{B}$ and $x \in X$,

$$
(b \triangleright x)^{[0]} \otimes_{R}(b \triangleright x)^{[1]}=b^{(1)}+\triangleright x^{[0]} \otimes_{R} b^{(2)} x^{[1]} b^{(1)}{ }_{-},
$$

where for the coproduct $\Delta$ and the inverse of the map (2.19) the respective index notations (with implicit summation), $\Delta(b)=b^{(1)} \otimes_{R} b^{(2)}$, and $\vartheta^{-1}\left(1_{\mathcal{B}} \otimes_{R} b\right)=b_{-} \otimes_{R^{o p}} b_{+}$are used, for $b \in \mathcal{B}$.

The anti Yetter-Drinfel'd module $X$ is said to be stable if in addition, for any $x \in X$,

$$
x^{[1]} \triangleright x^{[0]}=x .
$$

We need to show that condition (ii) in Definition 2.15 is meaningful, i.e. the expression on the right hand side of (2.21) is well defined. This follows by the following

Lemma 2.16. For an algebra $R$, consider a right $\times_{R}$-Hopf algebra $\mathcal{B}=(B, \xi, \zeta, \Delta, \epsilon)$. Let $X$ be a right $\mathcal{B}$-comodule and left $\mathcal{B}$-module. Keeping the notations in Definition 2.15, assume that axiom (i) in Definition 2.15 holds. Then the following hold.

(1) Considering $\mathcal{B}$ as a left $R$-module via $\zeta$, the $R$-module tensor product $X \otimes_{R} \mathcal{B}$ is a left $\mathcal{B}$-module via the action

$$
b^{\prime} \triangleright\left(x \otimes_{R} b\right):=b_{+}^{\prime} \triangleright x \otimes_{R} b b_{-}^{\prime} .
$$

(2) $X \otimes_{R} \mathcal{B}$ is a $\mathcal{B}-\mathcal{B}^{o p}$ bimodule, via the left $\mathcal{B}$-action in part (i) and the right $\mathcal{B}^{o p}$-action

$$
\left(x \otimes_{R} b\right) \triangleleft b^{\prime}:=x \otimes_{R} b^{\prime} b .
$$

(3) For any elements $x \in X$ and $r \in R$,

$$
\xi(r) \triangleright\left(x^{[0]} \otimes_{R} x^{[1]}\right)=\left(x^{[0]} \otimes_{R} x^{[1]}\right) \triangleleft \zeta(r) .
$$


Proof. By the first condition in (2.20) and Lemma 2.14 (ix), there is a well defined map

$$
\mathcal{B} \otimes_{\mathbb{K}}\left(X \otimes_{R} \mathcal{B}\right) \rightarrow X \otimes_{R} \mathcal{B}, \quad b^{\prime} \otimes_{\mathbb{K}}\left(x \otimes_{R} b\right) \mapsto b_{+}^{\prime} \triangleright x \otimes_{R} b b_{-}^{\prime} .
$$

It is an associative left $\mathcal{B}$-action by part (iii) of Lemma 2.14 and it is unital by part (iv). This proves claim (1). Claim (2) is obvious. Claim (3) follows by the following computation, for $r \in R$ and $x \in X$.

$$
\begin{aligned}
\xi(r) \triangleright\left(x^{[0]} \otimes_{R} x^{[1]}\right) & =\xi(r) x^{[0]} \otimes_{R} x^{[1]}=x^{[0][0]} \cdot \epsilon\left(\zeta(r) x^{[0][1]}\right) \otimes_{R} x^{[1]} \\
& =x^{[0]} \otimes_{R} x^{[1](2)} \zeta\left(\epsilon\left(\zeta(r) x^{[1](1)}\right)\right)=x^{[0]} \otimes_{R} \zeta(r) x^{[1]} \\
& =\left(x^{[0]} \otimes_{R} x^{[1]}\right) \triangleleft \zeta(r) .
\end{aligned}
$$

In the first equality we used that, by unitality and right $R$-module map property of the coproduct, the map (2.19) satisfies $\vartheta\left(1_{\mathcal{B}} \otimes_{R^{o p}} \xi(r)\right)=1_{\mathcal{B}} \otimes_{R} \xi(r)$. Hence $\xi(r)_{-} \otimes_{R^{o p}} \xi(r)_{+}=1_{\mathcal{B}} \otimes_{R^{o p}} \xi(r)$. The second equality follows by the second condition in (2.20). The third equality follows by coassociativity of the $\mathcal{B}$-coaction on $X$. The fourth equality follows by counitality of $\Delta$ and the right bialgebroid version of (2.3), i.e. the identity

$$
\Delta\left(\xi\left(r_{1}\right) \zeta\left(r_{2}\right) b \xi\left(r_{3}\right) \zeta\left(r_{4}\right)\right)=\zeta\left(r_{2}\right) b^{(1)} \zeta\left(r_{4}\right) \otimes_{R} \xi\left(r_{1}\right) b^{(2)} \xi\left(r_{3}\right),
$$

for $r_{1}, r_{2}, r_{3}, r_{4} \in R$ and $b \in \mathcal{B}$.

Using the notations in Lemma 2.16, the right hand side of (2.21) is equal to the well defined expression $b^{(1)} \triangleright\left(x^{[0]} \otimes_{R} x^{[1]}\right) \triangleleft b^{(2)}$.

REMARK 2.17. Note that a Hopf algebra $H$ over a commutative ring $\mathbb{K}$ is a $\times_{\mathbb{K}}$-Hopf algebra. The bialgebroid structure is given by the equal source and target maps $\mathbb{K} \rightarrow H, \kappa \mapsto \kappa 1_{H}$, and the coproduct and counit in $H$. The canonical map (2.19) has an inverse $\vartheta^{-1}\left(h^{\prime} \otimes_{\mathbb{K}} h\right)=$ $h^{\prime} S\left(h^{(1)}\right) \otimes_{\mathbb{K}} h^{(2)}$, where $h \mapsto h^{(1)} \otimes_{\mathbb{K}} h^{(2)}$ is the usual Sweedler index notation for the coproduct, with implicit summation understood. That is, $h_{-} \otimes_{\mathbb{K}} h_{+}=S\left(h^{(1)}\right) \otimes_{\mathbb{K}} h^{(2)}$. Clearly, in this case (2.20) becomes the trivial condition

$$
x \kappa=\left(\kappa 1_{H}\right) \triangleright x=\kappa x,
$$

for $\kappa \in \mathbb{K}$ and any element $x$ of a left $H$-module right $H$-comodule $X$. This condition simply expresses the requirement that the left and right $\mathbb{K}$-actions on $X$ are equal, and are induced by the $H$-module structure. The second condition (2.21) in Definition 2.15 reduces to

$$
(b \triangleright x)^{[0]} \otimes(b \triangleright x)^{[1]}=b^{(2)} \triangleright x^{[0]} \otimes b^{(3)} x^{[1]} S\left(b^{(1)}\right),
$$

for $b \in H$ and $x \in X$, which is the defining property of a (left-right) anti Yetter-Drinfel'd module $X$ of a Hopf algebra $H$ in [JŞ, Definition 4.1] or [HKRS1, Definition 2.1]. So we conclude that Definition 2.15 generalizes these definitions.

ExAmple 2.18. For an algebra $R$, consider a right $\times_{R}$-Hopf algebra $\mathcal{B}=(B, \xi, \zeta, \Delta, \epsilon)$. Note that left $B$-actions $\triangleright$ on $R$, satisfying $\zeta\left(r^{\prime}\right) \triangleright r=r r^{\prime}$, are in bijective correspondence with maps $\chi: B \rightarrow R$, obeying the following properties, for $r \in R, b, b^{\prime} \in B$.

$$
\chi(\zeta(r) b)=\chi(b) r \quad \chi\left(b b^{\prime}\right)=\chi\left(b \zeta\left(\chi\left(b^{\prime}\right)\right)\right), \quad \chi\left(1_{B}\right)=1_{R} .
$$

Indeed, in terms of such a map $\chi$, one can put $b \triangleright r:=\chi(b \zeta(r))$. Furthermore, right $B$-coactions on $R$, with underlying right regular $R$-module structure, are in bijective correspondence with grouplike elements in $B$, i.e. $g \in B$ such that $\Delta(g)=g \otimes_{R} g$ and $\epsilon(g)=1_{R}$. Indeed, in terms of a grouplike element $g$, a right $B$-coaction on $R$ is given by $r \mapsto 1_{R} \otimes_{R} g \xi(r)$. One checks that the left $B$-module determined by $\chi$ and the right $B$-comodule determined by $g$ combine into an anti Yetter-Drinfel'd module on $R$ if and only if, for $r \in R$ and $b \in B$,

$$
\epsilon(\xi(r) g)=\chi(\xi(r)), \quad \text { and } \quad g \xi(\chi(b))=b^{(2)} g b^{(1)}-\zeta\left(\chi\left(b^{(1)}+\right)\right) .
$$

The anti Yetter-Drinfel'd module $R$ is stable if in addition $\chi(g \xi(r))=r$, for all $r \in R$. The pair $(\chi, g)$ with these properties generalizes the notion of a modular pair in involution for a Hopf algebra in [CM01] or a weak Hopf algebra in [V]. 
Proposition 2.19. Let $\mathcal{B}$ be a right $\times_{R}$-Hopf algebra over an algebra $R$. Consider a right $\mathcal{B}$-module coring $C$ with $\mathcal{B}$-action $\triangleleft$.

(1) For an anti Yetter-Drinfel'd module $X$ of $\mathcal{B}$, the para-cyclic object in Theorem 2.11 projects to a para-cyclic structure on $C^{\otimes_{R} n+1} \otimes_{\mathcal{B}} X$.

(2) For a stable anti Yetter-Drinfel'd module $X$ of $\mathcal{B}$, the para-cyclic object $C^{\otimes_{R} n+1} \otimes_{\mathcal{B}} X$ in part (1) is cyclic, in which case it will be denoted by $Z_{*}(C, M)$.

Proof. We need to show that the composite map

$$
C^{\widehat{\otimes}_{R} n+1} \widehat{\otimes}_{R} X \stackrel{w_{n}}{\longrightarrow} C^{\widehat{\otimes}_{R} n+1} \widehat{\otimes}_{R} X \longrightarrow C^{\otimes_{R} n+1} \otimes_{\mathcal{B}} X
$$

is $\mathcal{B}$-balanced, i.e. that

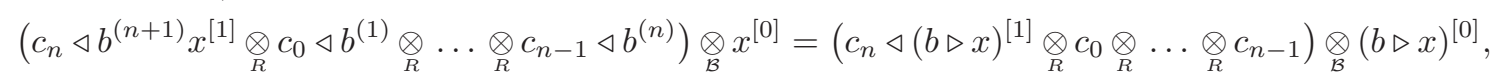

for $b \in \mathcal{B}, x \in X$ and $c_{0} \otimes_{R} \ldots \otimes_{R} c_{n} \in C^{\otimes_{R}{ }^{n+1}}$. By counitality of the coproduct in $\mathcal{B}$, the left hand side is equal to

$$
\begin{aligned}
c_{n} \triangleleft b^{(n+2)} & x^{[1]} \otimes_{R} c_{0} \triangleleft b^{(2)} \zeta\left(\epsilon\left(b^{(1)}\right)\right) \otimes_{R} c_{1} \triangleleft b^{(3)} \otimes_{R} \cdots \otimes_{R} c_{n-1} \triangleleft b^{(n+1)} \otimes_{\mathcal{B}} x^{[0]} \\
& =c_{n} \triangleleft b^{(n+2)} x^{[1]} \xi\left(\epsilon\left(b^{(1)}\right)\right) \otimes_{R} c_{0} \triangleleft b^{(2)} \otimes_{R} c_{1} \triangleleft b^{(3)} \otimes_{R} \cdots \otimes_{R} c_{n-1} \triangleleft b^{(n+1)} \otimes_{\mathcal{B}} x^{[0]} \\
& =c_{n} \triangleleft b^{(n+2)} x^{[1]} b^{(1)}{ }^{(1)} b^{(1)}+\otimes_{R} c_{0} \triangleleft b^{(2)} \otimes_{R} c_{1} \triangleleft b^{(3)} \otimes_{R} \cdots \otimes_{R} c_{n-1} \triangleleft b^{(n+1)} \otimes_{\mathcal{B}} x^{[0]} \\
& =c_{n} \triangleleft b_{+}{ }^{(n+2)} x^{[1]} b_{-} b_{+}{ }^{(1)} \otimes_{R} c_{0} \triangleleft b_{+}{ }^{(2)} \otimes_{R} \cdots \otimes_{R} c_{n-1} \triangleleft b_{+}{ }^{(n+1)} \otimes_{\mathcal{B}} x^{[0]} \\
& =\left(c_{n} \triangleleft b_{+}{ }^{(2)} x^{[1]} b_{-} \otimes_{R} c_{0} \otimes_{R} \cdots \otimes_{R} c_{n-1}\right) \triangleleft b_{+}{ }^{(1)} \otimes_{\mathcal{B}} x^{[0]} \\
& =\left(c_{n} \triangleleft b_{+}{ }^{(2)} x^{[1]} b_{-} \otimes_{R} c_{0} \otimes_{R} \cdots \otimes_{R} c_{n-1}\right) \otimes_{\mathcal{B}} b_{+}{ }^{(1)} \triangleright x^{[0]} \\
& =\left(c_{n} \triangleleft(b \triangleright x)^{[1]} \otimes_{R} c_{0} \otimes_{R} \cdots \otimes_{R} c_{n-1}\right) \otimes_{\mathcal{B}}(b \triangleright x)^{[0]} .
\end{aligned}
$$

The second equality follows by part (viii) in Lemma 2.14 and the third one follows by part (v). The last equality is a consequence of Lemma $2.14(\mathrm{v})$ and (2.21). This proves that the para-cyclic map $w_{n}$ in Theorem 2.11 factors to a map $\widehat{w}_{n}: C^{\otimes_{R} n+1} \otimes_{\mathcal{B}} X \rightarrow C^{\otimes_{R} n+1} \otimes_{\mathcal{B}} X$, hence it defines a paracyclic structure on the simplex in Lemma 2.12. This completes the proof of part (1). Furthermore, $\left(\widehat{w}_{n}\right)^{n+1}$ takes an element $\left(c_{0} \otimes_{R} \cdots \otimes_{R} c_{n}\right) \otimes_{\mathcal{B}} x \in C^{\otimes_{R} n+1} \otimes_{\mathcal{B}} X$ to

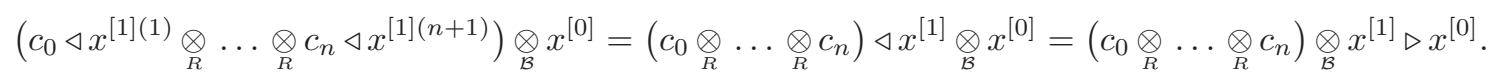

Hence if $X$ is a stable anti Yetter-Drinfel'd module, i.e. condition $(2.22)$ holds, then $\left(\widehat{w}_{n}\right)^{n+1}$ is the identity map. Thus we have claim (2) proven.

Remark 2.20. Let $\mathcal{B}=(B, \xi, \zeta, \Delta, \epsilon)$ be a right bialgebroid over an algebra $R$. Consider $\mathcal{B}$ as an $R^{o p}$-bimodule via right multiplications by $\xi$ and $\zeta$. This bimodule has an $R^{o p}$-coring structure with counit $\epsilon$ and coproduct $\Delta_{c o p}: b \mapsto b^{(2)} \otimes_{R^{o p}} b^{(1)}$, co-opposite to $\Delta$. Together with the opposite algebra $B^{o p}$, they constitute a left $R^{o p}$-bialgebroid $\mathcal{B}_{c o p}^{o p}=\left(B^{o p}, \xi, \zeta, \Delta_{c o p}, \epsilon\right)$. A right $\mathcal{B}$-module algebra $T$ determines a left $\mathcal{B}_{c o p}^{o p}$-module algebra $T^{o p}$, canonically. Furthermore, a right $\mathcal{B}$-comodule $X$ can be looked at as a left $\mathcal{B}_{c o p}^{o p}$-comodule. Application of Theorem 2.4 to the left $R^{o p}$-bialgebroid $\mathcal{B}_{c o p}^{o p}$, the left $\mathcal{B}_{c o p}^{o p}$-module algebra $T^{o p}$ and the left $\mathcal{B}_{c o p}^{o p}$-comodule $X$, yields a para-cocyclic cosimplex that has in degree $n$

$$
\left(T^{o p}\right)^{\widehat{\otimes}_{R^{o p} n+1}} \widehat{\otimes}_{R^{o p}} X \cong T^{\widehat{\otimes}_{R} n+1} \widehat{\otimes}_{R} X,
$$

where the isomorphism is given by reversing the order of the factors, i.e.

$$
t_{0} \widehat{\otimes}_{R^{o p}} t_{1} \widehat{\otimes}_{R^{o p}} \ldots \widehat{\otimes}_{R^{o p}} t_{n} \widehat{\otimes}_{R^{o p}} x \mapsto t_{n} \widehat{\otimes}_{R} t_{n-1} \widehat{\otimes}_{R} \ldots \widehat{\otimes}_{R} t_{0} \widehat{\otimes}_{R} x .
$$

Resulting coface and codegeneracy maps on $T \widehat{\otimes}_{R} \ldots \widehat{\otimes}_{R} T \widehat{\otimes}_{R} X$ are the maps in (2.2) and the para-cocyclic map comes out as

$$
t_{0} \widehat{\otimes}_{R} \ldots \widehat{\otimes}_{R} t_{n} \widehat{\otimes}_{R} x \quad \mapsto t_{n} \triangleleft x^{[1]} \widehat{\otimes}_{R} t_{0} \widehat{\otimes}_{R} \ldots \widehat{\otimes}_{R} t_{n-1} \widehat{\otimes}_{R} x^{[0]} .
$$

By the right $\mathcal{B}$-module map property of the maps $\varphi: R \rightarrow T, r \mapsto 1_{T} \triangleleft \xi(r)$, and the multiplication map $m_{T}: T \otimes_{R} T \rightarrow T$, the coface and codegeneracy maps (2.2) project to $T^{\otimes_{R} n+1} \otimes_{\mathcal{B}} X$. Note 
moreover that the para-cocyclic map (2.24) and the para-cyclic map (2.18) are of the same form. Hence it follows by the computation in the proof of Proposition 2.19 that also the para-cocyclic map (2.24) projects to $T^{\otimes_{R} n+1} \otimes_{\mathcal{B}} X$, whenever $X$ is an anti Yetter-Drinfel'd module of $\mathcal{B}$. That is, in this case $T^{\otimes_{R}{ }^{*+1}} \otimes_{\mathcal{B}} X$ is a para-cocyclic object, which is cocyclic if the anti Yetter-Drinfel'd module $X$ is stable.

2.D. Galois extensions of $\times_{R}$-Hopf algebras. For a right $\times_{R}$-Hopf algebra $\mathcal{B}$ over an algebra $R$, consider a right comodule algebra $T$ with coaction $t \mapsto t^{[0]} \otimes_{R} t^{[1]}$ (where implicit summation is understood). The subalgebra $S$ of coinvariants in $T$ consists of those elements $s \in T$ for which $s^{[0]} \otimes_{R} s^{[1]}=s \otimes_{R} 1_{\mathcal{B}}$. To the inclusion map $S \hookrightarrow T$ one can associate a cyclic simplex

$$
Z_{*}(T / S)=T^{\widehat{\otimes}_{S} *+1}
$$

as in Corollary 1.29. On the other hand, it follows by Proposition 2.19 that, regarding $\mathcal{B}$ as a right $\mathcal{B}$-module coring, for any stable anti Yetter-Drinfel'd module $X$ of $\mathcal{B}$ there is a cyclic simplex

$$
Z_{*}(B, X)=\mathcal{B}^{\otimes_{R} *+1} \otimes_{\mathcal{B}} X,
$$

where $\mathcal{B}^{\otimes_{R} n+1}$ is understood to be a right $\mathcal{B}$-module via the diagonal action. In this section, under the additional assumption that $T$ is a $\mathcal{B}$-Galois extension of $S$, we construct a stable anti Yetter-Drinfel'd module $X:=T /\{s t-t s \mid s \in S, t \in T\} \cong S \otimes_{S^{e}} T$ of $\mathcal{B}$, such that the cyclic simplices (2.25) and (2.26) are isomorphic. This extends [JŞ, Theorem 3.7].

In a right comodule algebra $T$ of a right bialgebroid $\mathcal{B}$ over an algebra $R$, we denote the coaction by $\varrho: t \mapsto t^{[0]} \otimes_{R} t^{[1]}$, where implicit summation is understood. For the iterated power of the coaction we write $\left(\varrho \otimes_{R} \mathcal{B}^{\otimes_{R} n-1}\right) \circ \cdots \circ\left(\varrho \otimes_{R} \mathcal{B}\right) \circ \varrho(t)=: t^{[0]} \otimes_{R} \cdots \otimes_{R} t^{[n-1]} \otimes_{R} t^{[n]}$.

Definition 2.21. Let $\mathcal{B}$ be a right bialgebroid over an algebra $R$. A right $\mathcal{B}$-comodule algebra $T$ is said to be a $\mathcal{B}$-Galois extension of its coinvariant subalgebra $S$ if the canonical map

$$
\text { can }: T \otimes_{S} T \rightarrow T \otimes_{R} \mathcal{B}, \quad t^{\prime} \otimes_{S} t \mapsto t^{\prime} t^{[0]} \otimes_{R} t^{[1]}
$$

is bijective.

For example, if $\mathcal{B}=(B, \xi, \zeta, \Delta, \epsilon)$ is a right $\times_{R}$-Hopf algebra, then the right regular $\mathcal{B}$-comodule algebra is a $\mathcal{B}$-Galois extension of the coinvariant subalgebra $\zeta(R) \cong R^{o p}$.

Let $\mathcal{B}$ be a right $R$-bialgebroid and $T$ a right $\mathcal{B}$-comodule algebra. It follows by the right $R$-module map property of a right $\mathcal{B}$-coaction and (2.13) that, for a coinvariant $s \in S$ and $r \in R$,

$$
\left(\left(1_{T} \cdot r\right) s\right)^{[0]} \otimes_{R}\left(\left(1_{T} \cdot r\right) s\right)^{[1]}=s \otimes_{R} \xi(r)=\left(s\left(1_{T} \cdot r\right)\right)^{[0]} \otimes_{R}\left(s\left(1_{T} \cdot r\right)\right)^{[1]} .
$$

Hence, applying the counit of $\mathcal{B}$ to the second factor on both sides, we conclude that the elements $s \in S$ commute with $1_{T} \cdot r$, for all $r \in R$. Hence $T \otimes_{R} \mathcal{B}$ is a right $S$-module, with action

$$
\left(t \otimes_{R} b\right) \cdot s:=t s \otimes_{R} b .
$$

Consider a right $\times_{R}$-Hopf algebra $\mathcal{B}$ over an algebra $R$, and a $\mathcal{B}$-Galois extension $S \subseteq T$. As in Lemma 2.14, in terms of the maps (2.19) and (2.27), introduce the index notations

$$
\operatorname{can}^{-1}\left(1_{T} \otimes_{R} b\right)=: b^{\{-\}} \otimes_{S} b^{\{+\}} \quad \text { and } \quad \vartheta^{-1}\left(1_{\mathcal{B}} \otimes_{R} b\right)=: b_{-} \otimes_{R^{o p}} b_{+},
$$

for $b \in \mathcal{B}$, where in both cases implicit summation is understood. The following lemma is a right bialgebroid version of [H, Lemma 4.1.21]. It extends Lemma 2.14 (vi).

Lemma 2.22. Consider a right $\times_{R}$-Hopf algebra $\mathcal{B}=(B, \xi, \zeta, \Delta, \epsilon)$ over an algebra $R$, and a $\mathcal{B}$ Galois extension $S \subseteq T$. Using the notations in (2.28), for any $b \in \mathcal{B}$ the following pentagonal equation holds in $\left(T \otimes_{R} \mathcal{B}\right) \otimes_{S} T$.

$$
b^{\{-\}[0]} \otimes_{R} b^{\{-\}[1]} \otimes_{S} b^{\{+\}}=b_{+}{ }^{\{-\}} \otimes_{R} b_{-} \otimes_{S} b_{+}{ }^{\{+\}} .
$$

Proof. The second condition in (2.12) implies that there is a well defined bijection

$$
\operatorname{can}_{13}:\left(T \otimes_{R} \mathcal{B}\right) \otimes_{S} T \rightarrow T \otimes_{R}\left(\mathcal{B} \otimes_{R^{o p}} \mathcal{B}\right), \quad\left(t^{\prime} \otimes_{R} b\right) \otimes_{S} t \mapsto t^{\prime} t^{[0]} \otimes_{R}\left(b \otimes_{R^{o p}} t^{[1]}\right),
$$

where in the $R^{o p}$-module tensor product $\mathcal{B} \otimes_{R^{o p}} \mathcal{B}$ the $R^{o p}$-actions $R^{o p} \otimes_{\mathbb{K}} \mathcal{B} \otimes_{\mathbb{K}} R^{o p} \rightarrow \mathcal{B}, r_{1} \otimes_{\mathbb{K}}$ $b \otimes_{\mathbb{K}} r_{2} \mapsto \zeta\left(r_{2}\right) b \zeta\left(r_{1}\right)$ are used, and $\mathcal{B} \otimes_{R^{o p}} \mathcal{B}$ is meant to be a left $R$-module via the action 
$r \cdot\left(b \otimes_{R^{o p}} b^{\prime}\right)=b \otimes_{R^{o p}} b^{\prime} \zeta(r)$. A straightforward computation using (2.13) shows that the $S$ bimodule map and right $R$-module map (2.27), and the left $R$-module map (2.19) satisfy

$$
\left(\operatorname{can} \otimes_{R} \mathcal{B}\right) \circ\left(T \otimes_{S} \operatorname{can}\right)=\left(T \otimes_{R} \vartheta\right) \circ \operatorname{can}_{13} \circ\left(\operatorname{can} \otimes_{S} T\right) .
$$

Hence, by bijectivity of all involved maps,

$$
\left(\operatorname{can} \otimes_{S} T\right) \circ\left(T \otimes_{S} \operatorname{can}^{-1}\right)=\operatorname{can}_{13}^{-1} \circ\left(T \otimes_{R} \vartheta^{-1}\right) \circ\left(\operatorname{can} \otimes_{R} \mathcal{B}\right) .
$$

Application of this identity to $1_{T} \otimes_{S} 1_{T} \otimes_{R} b$ yields the claim.

For an algebra extension $S \subseteq T, T$ has a canonical $S$-bimodule structure. Hence application of the functor $\Pi: S$-Mod- $S \rightarrow$ Mod- $\mathbb{K}$ in Definition 1.13 to $T$ yields a $\mathbb{K}$-module $\Pi T \cong S \widehat{\otimes}_{S} T$.

Proposition 2.23. Consider a right $\times_{R}$-Hopf algebra $\mathcal{B}$ over an algebra $R$, and a $\mathcal{B}$-Galois extension $S \subseteq T$. Then the quotient

$$
T_{S}:=S \widehat{\otimes}_{S} T
$$

is a stable anti Yetter-Drinfel'd module.

Proof. Since the $S$-, and $R$-actions on $T$ commute (cf. second paragraph following Definition 2.21), there is a unique $R$-bimodule structure on $T_{S}$ such that the epimorphism $p_{T}: T \rightarrow T_{S}$ is an $R$ bimodule map. Furthermore, by the $S$-bimodule map property of the coaction $\varrho: t \mapsto t^{[0]} \otimes_{R} t^{[1]}$ in $T$, the map $\left(p_{T} \otimes_{R} \mathcal{B}\right) \circ \varrho: T \rightarrow T_{S} \otimes_{R} \mathcal{B}$ coequalizes the left and right $S$-actions on $T$. Hence there exists a unique right $\mathcal{B}$-comodule structure on $T_{S}$ such that $p_{T}: T \rightarrow T_{S}$ is a right $\mathcal{B}$-comodule map.

The algebra map $S \hookrightarrow T$ equips $T$ with an $S$-bimodule structure. The center $\left(T \otimes_{S} T\right)^{S}$ of the $S$ bimodule $T \otimes_{S} T$ is an algebra, with multiplication $\left(\sum_{i} u_{i} \otimes_{S} u_{i}^{\prime}\right)\left(\sum_{j} v_{j} \otimes_{S} v_{j}^{\prime}\right)=\sum_{i, j} v_{j} u_{i} \otimes_{S} u_{i}^{\prime} v_{j}^{\prime}$. Recall from [JŞ, Section 2.2] that for any any $T$-bimodule $M$, the quotient $S \widehat{\otimes}_{S} M \cong M /\{s \cdot m-$ $m \cdot s \mid s \in S, m \in M\}$ is a left $\left(T \otimes_{S} T\right)^{S}$-module via the action

$$
\left(T \otimes_{S} T\right)^{S} \otimes_{\mathbb{K}} M_{S} \rightarrow M_{S}, \quad\left(\sum_{i} u_{i} \otimes_{S} u_{i}^{\prime}\right) \otimes_{\mathbb{K}} p_{M}(m) \mapsto \sum_{i} p_{M}\left(u_{i}^{\prime} m u_{i}\right),
$$

where $p_{M}: M \rightarrow S \widehat{\otimes}_{S} M$ denotes the canonical epimorphism. In particular, $T_{S}$ is a left $\left(T \otimes_{S} T\right)^{S}-$ module.

On the other hand, for a Galois extension $S \subseteq T$ by a right $R$-bialgebroid $\mathcal{B}$, using the notation in (2.28), the map

$$
\mathcal{B} \rightarrow\left(T \otimes_{S} T\right)^{S}, \quad b \mapsto b^{\{-\}} \otimes_{S} b^{\{+\}}
$$

is an algebra homomorphism. Indeed, by the $S$-bimodule map property of the coaction on $T$ it follows that

$$
\operatorname{can}\left(s b^{\{-\}} \otimes_{S} b^{\{+\}}\right)=s \otimes_{R} b=\operatorname{can}\left(b^{\{-\}} \otimes_{S} b^{\{+\}} s\right) .
$$

Hence, by bijectivity of can, $b^{\{-\}} \otimes_{S} b^{\{+\}} \in\left(T \otimes_{S} T\right)^{S}$, for all $b \in \mathcal{B}$. The map (2.30) is unital and multiplicative since by (2.13) for all $b, b^{\prime} \in \mathcal{B}$,

$$
\operatorname{can}\left(1_{T} \otimes_{S} 1_{T}\right)=1_{T} \otimes_{S} 1_{\mathcal{B}} \quad \text { and } \quad \operatorname{can}\left(b^{\prime\{-\}} b^{\{-\}} \otimes_{S} b^{\{+\}} b^{\prime\{+\}}\right)=1_{T} \otimes_{R} b b^{\prime}
$$

and can is bijective. This proves that $T_{S}$ is a left $\mathcal{B}$-module, with a so called Miyashita-Ulbrich type action

$$
b \triangleright p_{T}(t)=p_{T}\left(b^{\{+\}} t b^{\{-\}}\right) .
$$

It remains to check the compatibility conditions in Definition 2.15 between the $\mathcal{B}$-module and $\mathcal{B}$-comodule structures on $T_{S}$. It follows by the $R$-bimodule map property of (2.27) that

$$
\zeta(r)^{\{-\}} \otimes_{S} \zeta(r)^{\{+\}}=r \cdot 1_{T} \otimes_{S} 1_{T} \quad \text { and } \quad \xi(r)^{\{-\}} \otimes_{S} \xi(r)^{\{+\}}=1_{T} \otimes_{S} 1_{T} \cdot r .
$$

Hence

$\zeta(r) \triangleright p_{T}(t)=p_{T}\left(t\left(r \cdot 1_{T}\right)\right)=p_{T}(t \cdot r)=p_{T}(t) \cdot r$ and $\xi(r) \triangleright p_{T}(t)=p_{T}\left(\left(1_{T} \cdot r\right) t\right)=p_{T}(r \cdot t)=r \cdot p_{T}(t)$. 
Furthermore, for $b \in \mathcal{B}$ and $t \in T$,

$$
\begin{aligned}
\left(b \triangleright p_{T}(t)\right)^{[0]} \otimes_{R}\left(b \triangleright p_{T}(t)\right)^{[1]} & =p_{T}\left(b^{\{+\}[0]} t^{[0]} b^{\{-\}[0]}\right) \otimes_{R} b^{\{+\}[1]} t^{[1]} b^{\{-\}[1]} \\
& =p_{T}\left(b^{(1)\{+\}} t^{[0]} b^{(1)\{-\}[0]}\right) \otimes_{R} b^{(2)} t^{[1]} b^{(1)\{-\}[1]} \\
& =p_{T}\left(b^{(1)}+{ }^{\{+\}} t^{[0]} b^{(1)}+{ }^{\{-\}}\right) \otimes_{R} b^{(2)} t^{[1]} b^{(1)}- \\
& =b^{(1)}+\triangleright p_{T}(t)^{[0]} \otimes_{R} b^{(2)} p_{T}(t)^{[1]} b^{(1)}-.
\end{aligned}
$$

The first equality follows by (2.31), the second condition in (2.13) and the comodule map property of $p_{T}$. The second equality is a consequence of the right $\mathcal{B}$-comodule map property of $(2.27)$ hence of the map (2.30). The third equality is resulted by the application of Lemma 2.22. The last equality follows by (2.31) and the comodule map property of $p_{T}$ again. Thus we proved that $T_{S}$ is an anti Yetter-Drinfel'd module. Finally, by the comodule map property of $p_{T}$ and the identity

$$
t^{[0]} t^{[1]\{-\}} \otimes_{S} t^{[1]\{+\}}=\operatorname{can}^{-1}\left(\operatorname{can}\left(1_{T} \otimes_{S} t\right)\right)=1_{T} \otimes_{S} t, \quad \text { for } t \in T,
$$

it follows that

$$
p_{T}(t)^{[1]} \triangleright p_{T}(t)^{[0]}=t^{[1]} \triangleright p_{T}(t)^{[0]}=p_{T}\left(t^{[1]\{+\}} t^{[0]} t^{[1]\{-\}}\right)=p_{T}(t) .
$$

That is, the anti Yetter-Drinfel'd module $T_{S}$ is stable.

LEMma 2.24. For an algebra $R$ over a commutative ring $\mathbb{K}$, consider a right $R$-bialgebroid $\mathcal{B}$ and a $\mathcal{B}$-Galois extension $S \subseteq T$. Using the notation in (2.28), for any non-negative integer $n$ there exist $S$-bimodule isomorphisms $T^{\otimes_{S} n+1} \cong T \otimes_{R} \mathcal{B}^{\otimes_{R} n}$,

$$
\begin{aligned}
& \alpha_{n}\left(t_{0}{\underset{S}{S}}_{\infty} \ldots{\underset{S}{S}}_{n} t_{n}\right)=t_{0} t_{1}^{[0]} t_{2}^{[0]} \ldots t_{n}^{[0]} \otimes_{R} t_{1}^{[1]} t_{2}^{[1]} \ldots t_{n}^{[1]} \otimes_{R} t_{2}^{[2]} t_{3}^{[2]} \ldots t_{n}^{[2]}{\underset{R}{\otimes}}_{R} \ldots{\underset{R}{R}}_{n-1}^{[n-1]} t_{n}^{[n-1]} \otimes_{R} t_{n}^{[n]}, \\
& \alpha_{n}^{-1}\left(t \otimes_{R}^{\otimes} b_{1} \otimes_{R} \cdots \otimes_{R}^{\otimes} b_{n}\right)=t b_{1}^{\{-\}} \otimes_{S} b_{1}^{\{+\}} b_{2}{ }^{\{-\}} \otimes_{S} b_{2}^{\{+\}} b_{3}{ }^{\{-\}} \otimes_{S} \cdots \otimes_{S}^{\otimes} b_{n-1}{ }^{\{+\}} b_{n}{ }^{\{-\}} \otimes_{S} b_{n}{ }^{\{+\}} \text {. }
\end{aligned}
$$

Projections of the above isomorphisms yield $\mathbb{K}$-module isomorphisms $\widehat{\alpha}_{n}: T^{\widehat{\otimes}_{S} n+1} \rightarrow T_{S} \otimes_{R} \mathcal{B}^{\otimes_{R} n}$, where $T_{S}$ is the $R$-bimodule (2.29).

Proof. It follows by the $S$-bimodule map property of the right $\mathcal{B}$-coaction on $T$ that $\alpha_{n}$ is a well defined $S$-bimodule map. The to-be-inverse $\alpha_{n}^{-1}$ is well defined by (2.32) and Lemma 2.14 (iii). We prove by induction that the maps $\alpha_{n}$ and $\alpha_{n}^{-1}$ are mutual inverses. For $n=0$, both $\alpha_{0}$ and $\alpha_{0}^{-1}$ are equal to the identity map on $T$, hence they are mutual inverses. It follows by the second condition in (2.13) that, for all values of $n$,

$$
\alpha_{n+1}=\left(\alpha_{n} \otimes_{R} \mathcal{B}\right) \circ\left(T^{\otimes_{S} n} \otimes_{S} \text { can }\right) \quad \text { and } \quad \alpha_{n+1}^{-1}=\left(T^{\otimes_{S} n} \otimes_{S} \mathrm{can}^{-1}\right) \circ\left(\alpha_{n}^{-1} \otimes_{R} \mathcal{B}\right) .
$$

Hence if $\alpha_{n}^{-1}$ is the inverse of $\alpha_{n}$ then $\alpha_{n+1}$ is also an $S$-bimodule isomorphism with inverse $\alpha_{n+1}^{-1}$. Applying the functor in Definition 1.13, from the category of $S$-bimodules to the category of $\mathbb{K}$ modules, it takes $\alpha_{n}$ to the required $\mathbb{K}$-module isomorphism $\widehat{\alpha}_{n}: T^{\widehat{\otimes}_{S} n+1} \rightarrow T_{S} \otimes_{R} \mathcal{B}^{\otimes_{R} n}$.

Lemma 2.25. Let $\mathcal{B}$ be a right $\times_{R}$-Hopf algebra over an algebra $R$. For the inverse of the canonical map (2.19) use the index notation in (2.28). Then, for any non-negative integer $n$, there exist right $\mathcal{B}$-module isomorphisms $\mathcal{B}^{\otimes_{R} n} \otimes_{R^{o p}} \mathcal{B} \cong \mathcal{B}^{\otimes_{R}}{ }^{n+1}$,

$$
\begin{aligned}
& \beta_{n}\left(b_{1} \otimes_{R} \cdots \otimes_{R} b_{n} \otimes_{R^{o p}} b^{\prime}\right)=b_{1} b^{(1)} \otimes_{R} \cdots \otimes_{R} b_{n} b^{\prime(n)} \otimes_{R} b^{(n+1)} \\
& \beta_{n}^{-1}\left(b_{1} \otimes_{R} \cdots \otimes_{R} b_{n} \otimes_{R} b^{\prime}\right)=b_{1} b_{-}^{\prime(1)} \otimes_{R} \cdots \otimes_{R} b_{n} b_{-}^{\prime(n)} \otimes_{R^{o p}} b_{+}^{\prime} .
\end{aligned}
$$

Proof. For any right $\mathcal{B}$-module $N$, the $\mathcal{B}$-bimodule isomorphism (2.19) induces a right $\mathcal{B}$-module isomorphism $N \otimes_{\mathcal{B}} \vartheta: N \otimes_{\mathcal{B}} \mathcal{B} \otimes_{R^{o p}} \mathcal{B} \cong N \otimes_{R^{o p}} \mathcal{B} \rightarrow N \otimes_{\mathcal{B}} \mathcal{B} \otimes_{R} \mathcal{B} \cong N \otimes_{R} \mathcal{B}$. Consider $\mathcal{B}^{\otimes_{R} n}$ as a right $\mathcal{B}$-module via the diagonal action. Then $\beta_{n}=\mathcal{B}^{\otimes_{R} n} \otimes_{\mathcal{B}} \vartheta$ is a right $\mathcal{B}$-module isomorphism as stated. 
THEOREM 2.26. Let $\mathcal{B}$ be a right $\times_{R}$-Hopf algebra over an algebra $R$ and let $S \subseteq T$ be a $\mathcal{B}$ Galois extension. Consider the right regular $\mathcal{B}$-module coring and the stable anti Yetter-Drinfel'd module $T_{S}$ in Proposition 2.23. Then the associated cyclic simplex $Z_{*}\left(B, T_{S}\right)$ in Proposition 2.19 is isomorphic to the cyclic simplex $Z_{*}(T / S)$ in Corollary 1.29.

Proof. In terms of the maps in Lemmata 2.24 and 2.25, for any non-negative integer $n$, one constructs a $\mathbb{K}$-module isomorphism $\omega_{n}$ as the composition of the following morphisms

$$
T^{\widehat{\otimes}_{S} n+1} \stackrel{\widehat{\alpha}_{n}}{\longrightarrow} T_{S} \underset{R}{\otimes} \mathcal{B}^{\otimes_{R} n} \longrightarrow \mathcal{B}^{\otimes_{R} n} \underset{R^{o p}}{\otimes} T_{S} \stackrel{\cong}{\longrightarrow} \mathcal{B}^{\otimes_{R} n} \underset{R^{o p}}{\otimes} \mathcal{B} \underset{\mathcal{B}}{\otimes} T_{S} \stackrel{\beta_{n} \otimes_{\mathcal{B}} T_{S}}{\longrightarrow} \mathcal{B}^{\otimes_{R} n+1} \underset{\mathcal{B}}{\otimes} T_{S}
$$

Explicitly,

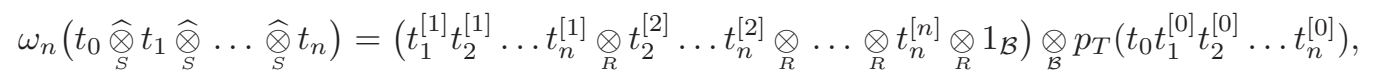

where $p_{T}: T \rightarrow T_{S} \cong S \widehat{\otimes}_{S} T$ denotes the canonical epimorphism. We show that (2.34) is a homomorphism of cyclic simplices.

Denote the counit in $\mathcal{B}$ by $\epsilon$. By multiplicativity of the coproduct in $\mathcal{B}$, for any integer $0 \leq k<n$,

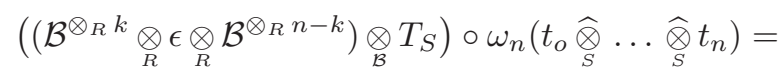

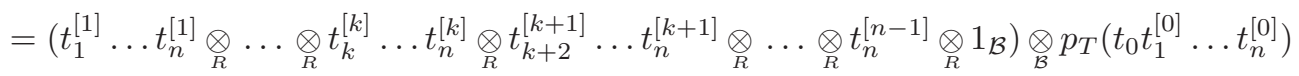

$$
\begin{aligned}
& =\omega_{n-1}\left(t_{o} \underset{S}{\widehat{\otimes}} \ldots \underset{S}{\widehat{\otimes}} t_{k-1} \underset{S}{\widehat{\otimes}} t_{k} t_{k+1} \underset{S}{\widehat{\otimes}} t_{k+2} \underset{S}{\widehat{\otimes}} \ldots \underset{S}{\widehat{\otimes}} t_{n}\right) \text {. }
\end{aligned}
$$

Furthermore, using the form of the (diagonal) right $\mathcal{B}$-action on $\mathcal{B}^{\otimes_{R}} n$ (in the second equality) and the form of the left $\mathcal{B}$-action (2.31) on $T_{S}$ (in the third equality), one computes

$$
\begin{aligned}
& \left(\left(\mathcal{B}^{\otimes_{R} n} \underset{R}{\otimes} \epsilon\right) \underset{\mathcal{B}}{\otimes} T_{S}\right) \circ \omega_{n}\left(t_{o} \underset{S}{\widehat{\otimes}} \ldots \underset{S}{\widehat{\otimes}} t_{n}\right)= \\
& =\left(t_{1}^{[1]} \ldots t_{n}^{[1]} \otimes_{R} t_{2}^{[2]} \ldots t_{n}^{[2]} \otimes_{R} \ldots \otimes_{R} t_{n}^{[n]}\right){\underset{\mathcal{B}}{B}}_{p_{T}}\left(t_{0} t_{1}^{[0]} \ldots t_{n}^{[0]}\right) \\
& =\left(t_{1}^{[1]} \ldots t_{n-1}^{[1]} \underset{R}{\otimes} t_{2}^{[2]} \ldots t_{n-1}^{[2]} \underset{R}{\otimes} \ldots{\underset{R}{\otimes}}_{n-1}^{[n-1]} \otimes_{R} 1_{\mathcal{B}}\right) \triangleleft t_{n}^{[1]}{\underset{\mathcal{B}}{\otimes}}_{\otimes_{T}}\left(t_{0} t_{1}^{[0]} \ldots t_{n}^{[0]}\right) \\
& =\left(t_{1}^{[1]} \ldots t_{n-1}^{[1]}{\underset{R}{R}}_{2}^{[2]} \ldots t_{n-1}^{[2]}{\underset{R}{R}}_{R} \ldots \otimes_{R} t_{n-1}^{[n-1]} \otimes_{R} 1_{\mathcal{B}}\right){\underset{\mathcal{B}}{\mathcal{B}}}_{T} p_{T}\left(t_{n}^{[1]\{+\}} t_{0} t_{1}^{[0]} \ldots t_{n}^{[0]} t_{n}^{[1]\{-\}}\right) \\
& =(t_{1}^{[1]} \ldots t_{n-1}^{[1]}{\underset{R}{R}}_{2}^{[2]} \ldots t_{n-1}^{[2]}{\underset{R}{R}}_{\ldots} \ldots{\underset{R}{R-1}}_{R}^{[n-1]} \underbrace{}_{R} 1_{\mathcal{B}}) \underset{\mathcal{B}}{\otimes} p_{T}\left(t_{n} t_{0} t_{1}^{[0]} \ldots t_{n-1}^{[0]}\right) \\
& =\omega_{n-1}\left(t_{n} t_{o} \underset{S}{\widehat{\otimes}} t_{1} \widehat{\bigotimes}_{S} \ldots \widehat{\bigotimes}_{S} t_{n-1}\right),
\end{aligned}
$$

where the penultimate equality follows by (2.33). This proves that the map $\omega_{n}$ is compatible with the face maps.

Denote the coproduct in $\mathcal{B}$ by $\Delta$. It follows by its multiplicativity that, for any integer $0 \leq k<n$,

$$
\begin{aligned}
& \left(\left(\mathcal{B}^{\otimes_{R} k} \otimes_{R} \Delta \underset{R}{\otimes_{1}} \mathcal{B}_{R} n-k\right) \underset{\mathcal{B}}{\otimes} T_{S}\right) \circ \omega_{n}\left(t_{o} \underset{S}{\widehat{\otimes}} \ldots \widehat{\bigotimes}_{S} t_{n}\right)= \\
& =\left(t_{1}^{[1]} \ldots t_{n}^{[1]} \otimes_{R} \ldots{\underset{R}{R}}_{k+1}^{[k+1]} \ldots t_{n}^{[k+1]} \otimes_{R} t_{k+1}^{[k+2]} \ldots t_{n}^{[k+2]} \otimes_{R} \ldots{\underset{R}{\otimes}}_{n}^{[n+1]}{\underset{R}{R}}_{\mathcal{B}}\right){\underset{\mathcal{B}}{B}}_{T} p_{T}\left(t_{0} t_{1}^{[0]} \ldots t_{n}^{[0]}\right) \\
& =\omega_{n+1}\left(t_{o} \underset{S}{\widehat{\otimes}} \ldots \widehat{S}_{S}^{\widehat{\otimes}} t_{k} \underset{S}{\widehat{\otimes}} 1_{T} \underset{S}{\widehat{\otimes}} t_{k+1} \underset{S}{\widehat{\otimes}} \ldots \underset{S}{\widehat{\otimes}} t_{n}\right) \text {. }
\end{aligned}
$$

Furthermore, by unitality of $\Delta$ and of the $\mathcal{B}$-coaction on $T$,

$$
\begin{aligned}
& \left(\left(\mathcal{B}^{\otimes_{R} n} \underset{R}{\otimes} \Delta\right) \underset{\mathcal{B}}{\otimes} T_{S}\right) \circ \omega_{n}\left(t_{o} \underset{S}{\widehat{\otimes}} \cdots \underset{S}{\widehat{\otimes}} t_{n}\right)=
\end{aligned}
$$

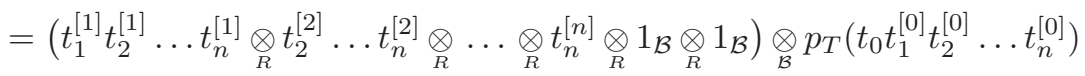

$$
\begin{aligned}
& =\omega_{n+1}\left(t_{o} \underset{S}{\widehat{\otimes}} \ldots \underset{S}{\widehat{\otimes}} t_{n} \underset{S}{\widehat{\otimes}} 1_{T}\right) \text {. }
\end{aligned}
$$

This proves that the map $\omega_{n}$ is compatible with the degeneracy maps. 
Finally, by similar steps as in (2.35), the cyclic map $\widehat{w}_{n}$ in Proposition 2.19 is checked to satisfy

$$
\begin{aligned}
& \widehat{w}_{n} \circ \omega_{n}\left(t_{o} \widehat{\otimes}_{S} \ldots \widehat{\otimes}_{S} t_{n}\right)=
\end{aligned}
$$

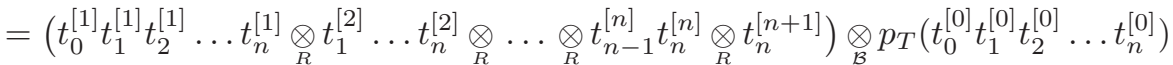

$$
\begin{aligned}
& =\left(t_{0}^{[1]} t_{1}^{[1]} t_{2}^{[1]} \ldots t_{n-1}^{[1]} \underset{R}{\otimes} t_{1}^{[2]} \ldots t_{n-1}^{[2]}{\underset{R}{R}}_{R} \ldots{\underset{R}{\otimes}}_{n-1}^{[n]}{ }_{R}^{\otimes} 1_{\mathcal{B}}\right) \underset{\mathcal{B}}{\otimes} p_{T}\left(t_{n} t_{0}^{[0]} t_{1}^{[0]} t_{2}^{[0]} \ldots t_{n-1}^{[0]}\right) \\
& =\omega_{n}\left(t_{n} \underset{S}{\widehat{\otimes}} t_{0} \underset{S}{\widehat{\otimes}} \ldots \widehat{\otimes}_{S} t_{n-1}\right) \text {. }
\end{aligned}
$$

Hence $\omega_{n}$ is compatible with the cyclic maps as well, what proves the claim.

\section{CyClic homology of Groupoids}

Consider a groupoid $\mathcal{G}$ (i.e. a small category in which all morphisms are invertible) with a finite set $\mathcal{G}^{0}$ of objects and an arbitrary set $\mathcal{G}^{1}$ of morphisms. Via the map associating to $x \in \mathcal{G}^{0}$ the identity morphism $x \rightarrow x$, we consider $\mathcal{G}^{0}$ as a subset of $\mathcal{G}^{1}$. Composition in $\mathcal{G}$ is denoted by o while the source and target maps $\mathcal{G}^{1} \rightarrow \mathcal{G}^{0}$ are denoted by $s$ and $t$, respectively. For any field $\mathbb{K}$, the $\mathbb{K}$-vector space $B:=\mathbb{K} \mathcal{G}^{1}$, spanned by the elements of $\mathcal{G}^{1}$, has a right $\times_{R}$-Hopf algebra structure over the commutative base algebra $R:=\mathbb{K} \mathcal{G}^{0}$. Structure maps are the following. Multiplication in the $\mathbb{K}$-algebra $B$ is given on basis elements $g, g^{\prime} \in \mathcal{G}^{1}$ by $g \circ g^{\prime}$, if $g$ and $g^{\prime}$ are composable, i.e. $s(g)=t\left(g^{\prime}\right)$, and zero otherwise. We denote by juxtaposition this product, linearly extended to all elements of $B$. Unit element is $1_{B}=\sum_{x \in \mathcal{G}^{0}} x$. Similarly, $R$ is a commutative $\mathbb{K}$-algebra with minimal orthogonal idempotents $\left\{x \in \mathcal{G}^{0}\right\}$. Both algebra maps $\xi$ and $\zeta: R \rightarrow B$ are induced by the inclusion map $\mathcal{G}^{0} \hookrightarrow \mathcal{G}^{1}$. That is, $B$ is an $R$-module (or $R$-bimodule, with coinciding left and right actions) via multiplication on the right. Coproduct is diagonal on the basis elements $g \in \mathcal{G}^{1}$, i.e. $\Delta(g):=g \otimes_{R} g$. Counit $\epsilon$ maps $g \in \mathcal{G}^{1}$ to $s(g) \in \mathcal{G}^{0}$. The canonical map (2.19) has the explicit form on the generating set $\left\{g \otimes_{R} g^{\prime} \mid g, g^{\prime} \in \mathcal{G}^{1}\right\}$,

$$
\vartheta: B \otimes_{R} B \rightarrow B \otimes_{R} B, \quad g \otimes_{R} g^{\prime} \mapsto g g^{\prime} \otimes_{R} g^{\prime} .
$$

(Note that $R$-module structures in the domain and codomain are different, cf. Definition 2.13.) It obviously has an inverse $\vartheta^{-1}\left(g \otimes_{R} g^{\prime}\right)=g g^{\prime-1} \otimes_{R} g^{\prime}$.

In this final section we apply the theory developed in the earlier sections to the groupoid bialgebroid $\mathcal{B}$ and its stable anti Yetter-Drinfel'd modules. In this way, we obtain expressions for Hochschild and cyclic homologies of a groupoid with finitely many objects. Describing then any groupoid as a direct limit of groupoids with finitely many objects, we extend the computation of cyclic homology to arbitrary groupoids. Similar arguments don't seem to apply in case of Hochschild homology.

3.A. Anti Yetter-Drinfel'd modules for groupoids. The subject of this section is a complete characterization of (stable) anti Yetter-Drinfel'd modules of a groupoid bialgebroid. As a first step, we study comodules.

Proposition 3.1. Let $\mathcal{G}$ be a small groupoid with finite set of objects. Let $\mathcal{B}$ be the groupoid $R$ bialgebroid associated to $\mathcal{G}$. Then any right $\mathcal{B}$-comodule $M$ has a direct sum decomposition $M \cong$ $\oplus_{g \in \mathcal{G}^{1}} M_{g}$ (as an $R$-module) such that the $R$-action - and the $\mathcal{B}$-coaction @ satisfy the conditions

(i) $m \cdot x=\delta_{x, s(g)} m$ and

(ii) $\varrho(m)=m \otimes_{R} g$,

for $x \in \mathcal{G}^{0}, g \in \mathcal{G}^{1}$ and $m \in M_{g}$. Conversely, on an $R$-module $M \cong \oplus_{g \in \mathcal{G}^{1}} M_{g}$, which is subject to condition (i), there is a unique right $\mathcal{B}$-coaction satisfying (ii).

Proof. Recall that by definition $R$ acts on $B$ by right multiplication. For any $g \in \mathcal{G}^{1}$ there is an $R$-module map $\chi_{g}: B \rightarrow R, h \mapsto \delta_{g, h} s(g)$. Introduce the map

$$
\pi_{g}:=\left(M \otimes_{R} \chi_{g}\right) \circ \varrho: M \rightarrow M .
$$

We claim that $M$ is isomorphic to a direct sum of the $R$-modules $M_{g}:=\operatorname{Im}\left(\pi_{g}\right)$. Since $B$ is a free $\mathbb{K}$-module, there exist (non-unique) elements $\left\{m_{g} \mid g \in \mathcal{G}^{1}\right\}$ in $M$, in terms of which $\varrho(m)=\sum_{g \in \mathcal{G}^{1}} m_{g} \otimes_{R} g$, hence $\pi_{g}(m)=m_{g} \cdot s(g)$. By construction, for a given element $m \in M$ 
there are only finitely many elements $g \in \mathcal{G}^{1}$ such that $m_{g} \neq 0$, hence $\pi_{g}(m) \neq 0$. By coassociativity of $\varrho$,

$$
\sum_{g, h \in \mathcal{G}^{1}}\left(m_{h}\right)_{g} \otimes_{R} g \otimes_{R} h=\sum_{g \in \mathcal{G}^{1}} m_{g} \otimes_{R} g \otimes_{R} g .
$$

Hence applying $M \otimes_{R} \chi_{g^{\prime}} \otimes_{R} \chi_{h^{\prime}}$, for some $g^{\prime}, h^{\prime} \in \mathcal{G}^{1}$, we conclude that $\left(m_{h^{\prime}}\right)_{g^{\prime}} \cdot s\left(g^{\prime}\right) s\left(h^{\prime}\right)=$ $\delta_{g^{\prime}, h^{\prime}} m_{g^{\prime}} \cdot s\left(g^{\prime}\right)$, i.e. $\pi_{g^{\prime}} \circ \pi_{h^{\prime}}=\delta_{g^{\prime}, h^{\prime}} \pi_{g^{\prime}}$. By counitality of $\varrho$, for all $m \in M$,

$$
\sum_{g \in \mathcal{G}^{1}} \pi_{g}(m)=\sum_{g \in \mathcal{G}^{1}} m_{g} \cdot s(g)=m
$$

This proves the direct sum decomposition of $M$ as an $R$-module. Condition (i) follows by the computation, for $m \in M, g \in \mathcal{G}^{1}$ and $x \in \mathcal{G}^{0}$,

$$
\pi_{g}(m) \cdot x=m_{g} \cdot s(g) x=\delta_{s(g), x} m_{g} \cdot s(g)=\delta_{s(g), x} \pi_{g}(m) .
$$

Moreover, for $m \in M$,

$$
\varrho(m)=\sum_{g \in \mathcal{G}^{1}} m_{g} \otimes_{R} g=\sum_{g \in \mathcal{G}^{1}} m_{g} \otimes_{R} g s(g)=\sum_{g \in \mathcal{G}^{1}} m_{g} \cdot s(g) \otimes_{R} g=\sum_{g \in \mathcal{G}^{1}} \pi_{g}(m) \otimes_{R} g .
$$

Hence, by orthogonality of the projections $\pi_{g}$,

$$
\varrho\left(\pi_{h}(m)\right)=\sum_{g \in \mathcal{G}^{1}} \pi_{g}\left(\pi_{h}(m)\right) \otimes_{R} g=\pi_{h}(m) \otimes_{R} h,
$$

which proves condition (ii). Conversely, assume that for an $R$-module $M \cong \oplus_{g \in \mathcal{G}^{1}} M_{g}$ condition (i) holds. Put $\varrho_{g}: M_{g} \rightarrow M_{g} \otimes_{R} B, m \mapsto m \otimes_{R} g$. One can check that it makes $M_{g}$ to a right $\mathcal{B}$-comodule. By universality of a direct sum, this defines a unique right $\mathcal{B}$-coaction $\varrho$ on $M$, such that condition (ii) holds.

Note that property (ii) in Proposition 3.1 characterizes uniquely the elements $m$ of a component $M_{g}$. Indeed, if for $m \in M, \varrho(m)=m \otimes_{R} g$, then by counitality of the coaction $\varrho$ we obtain $\pi_{g}(m)=m \cdot s(g)=m$.

We are ready to characterize (stable) anti Yetter-Drinfel'd modules of groupoids.

THEOREM 3.2. Let $\mathcal{G}$ be a small groupoid with finite set of objects. Let $\mathcal{B}$ be the groupoid bialgebroid associated to $\mathcal{G}$. A left $\mathcal{B}$-module $M \cong \oplus_{g \in \mathcal{G}^{1}} M_{g}$, with action $\triangleright$, is an anti Yetter-Drinfel'd $\mathcal{B}$ module if and only if the following conditions hold.

(i) For $x \in \mathcal{G}^{0}, g \in \mathcal{G}^{1}$ and $m \in M_{g}, \delta_{x, s(g)} m=x \triangleright m=\delta_{x, t(g)} m$.

(ii) For $g, h \in \mathcal{G}^{1}$ and $m \in M_{g}$, the element $h \triangleright m$ is zero if $h g h^{-1}=0$ in $B$ and it belongs to $M_{h g h^{-1}}$ if $h g h^{-1} \neq 0$ in $B$.

The anti Yetter-Drinfel'd $\mathcal{B}$-module $M$ is stable if and only if in addition $g \triangleright m=m$, for all $g \in \mathcal{G}^{1}$ and $m \in M_{g}$.

Proof. By Proposition 3.1 there is a unique $\mathcal{B}$-coaction on $M$ corresponding to the given direct sum decomposition. For $g \in \mathcal{G}^{1}$, it takes $m \in M_{g}$ to $m \otimes_{R} g$. The $R$-bimodule structure corresponding to this coaction comes out, for $x, y \in \mathcal{G}^{0}, g \in \mathcal{G}^{1}$ and $m \in M_{g}$, as

$$
x \cdot m \cdot y=m \cdot \epsilon(x g y)=\delta_{s(g), y} \delta_{t(g), x} m \cdot s(g)=\delta_{s(g), y} \delta_{t(g), x} m .
$$

Hence axiom (2.20) of an anti Yetter-Drinfel'd module translates to condition (i) in the theorem. A straightforward computation shows that for a groupoid bialgebroid, axiom (2.21) of an anti Yetter-Drinfel'd module takes the form

$$
\varrho(h \triangleright m)=h \triangleright m \otimes_{R} h g h^{-1},
$$

for $h, g \in \mathcal{G}^{1}$ and $m \in M_{g}$. If $h g h^{-1}=0$ in $B$, then the right hand side of (3.1) vanishes. Since $\varrho$ is a monomorphism of $R$-modules (split by $M \otimes_{R} \epsilon$ ), this is equivalent to $h \triangleright m=0$. If $h g h^{-1} \neq 0$ in $B$, then (3.1) is equivalent to $h \triangleright m \in M_{h g h^{-1}}$ (cf. Proposition 3.1, and discussions following it). This completes the proof. 
As a consequence of Theorem 3.2 (condition (i)), in the direct sum decomposition of an anti Yetter-Drinfel'd module $M$ of a groupoid bialgebroid, $M_{g}$ is non-zero for only those elements $g \in \mathcal{G}^{1}$ for which $s(g)=t(g)$, i.e. which are loops. That is, introducing the notation $\mathcal{L}(\mathcal{G}):=\{g \in$ $\left.\mathcal{G}^{1} \mid s(g)=t(g)\right\}$, one can write $M \cong \oplus_{l \in \mathcal{L}(\mathcal{G})} M_{l}$.

Our next aim is to decompose an anti Yetter-Drinfel'd module $M$ of a groupoid bialgebroid $\mathcal{B}$ as a direct sum of anti Yetter-Drinfel'd modules. For a loop $l \in \mathcal{L}(\mathcal{G})$, denote by $[l]$ the orbit of $l$ in $\mathcal{L}(\mathcal{G})$ for the adjoint action, that is, the set of different non-zero elements of the form $g l g^{-1}$, as $g$ runs through $\mathcal{G}^{1}$. This gives a ( $\mathcal{G}$-invariant) partition $\mathcal{T}(\mathcal{G})$ of $\mathcal{L}(\mathcal{G})$. Using Theorem 3.2 one concludes that $M_{[l]}:=\oplus_{l^{\prime} \in[l]} M_{l^{\prime}}$ is an anti Yetter Drinfel'd $\mathcal{B}$-module, and

$$
M \cong \bigoplus_{[l] \in \mathcal{T}(\mathcal{G})} M_{[l]},
$$

as anti Yetter-Drinfel'd modules. Let us give an alternative description of the anti Yetter-Drinfel'd module $M_{[l]}$. Introduce the following subalgebras of the groupoid algebra $B$. For $l \in \mathcal{L}(\mathcal{G})$, let $B_{l}$ be the group algebra of the centralizer $\mathcal{G}_{l}^{1}$ of $l$ in the group $\left\{l^{\prime} \in \mathcal{L}(\mathcal{G}) \mid s\left(l^{\prime}\right)=s(l)\right\}$. That is,

$$
B_{l}:=\mathbb{K} \mathcal{G}_{l}^{1} \equiv \mathbb{K}\left\{l^{\prime} \in \mathcal{L}(\mathcal{G}) \mid l^{\prime} l l^{\prime-1}=l\right\} .
$$

The algebra $B_{l}$ possesses a unit (the element $s(l)=t(l)$ ), different from the unit element of $B$. For $x \in \mathcal{G}^{0}$, let $B(x)$ denote the vector space spanned by the elements $g \in \mathcal{G}^{1}$, such that $s(g)=x$. Consider the group bialgebra structure of $B_{l}$. Clearly, for any $l \in \mathcal{L}(\mathcal{G})$, the component $M_{l}$ in the direct sum decomposition of an anti Yetter Drinfel'd $\mathcal{B}$-module $M$ is an anti Yetter-Drinfel'd module of the group bialgebra $B_{l}$ (in the sense of [JS], cf. Theorem 3.2). Moreover, $B(s(l)$ ) is a $B$ - $B_{l}$ bimodule and a right $B_{l}$-module coalgebra (with coproduct induced by the map $g \mapsto g \otimes_{\mathbb{K}} g$, for $g \in \mathcal{G}^{1}$ such that $s(g)=s(l)$.)

LEMma 3.3. Let $\mathcal{G}$ be a small groupoid with a finite set of objects. Let $\mathcal{B}$ be the groupoid bialgebroid over a field $\mathbb{K}$, associated to $\mathcal{G}$. Keeping the notation introduced above, $B(s(l))$ is free as a right $B_{l}$-module, for any $l \in \mathcal{L}(\mathcal{G})$.

Proof. Since any morphism in a groupoid is invertible, the right action of the group $\mathcal{G}_{l}^{1}$ on the set $\left\{g \in \mathcal{G}^{1} \mid s(g)=s(l)\right\}$ is faithful in the sense that $g l^{\prime}=g$ implies $l^{\prime}=s(l)$. Hence the claim follows by the fact that $\mathbb{K} X$ is a free module for a group algebra $\mathbb{K} G$ whenever $G$ acts faithfully on the set $X$. Indeed, fix a $\mathbb{K}$-basis $\left\{e_{x} \mid x \in X\right\}$ of $\mathbb{K} X$, such that $e_{x} \cdot g=e_{x \cdot g}$. Fix a section $f$ of the canonical epimorphism from $X$ to the set of $G$-orbits $X / G$. By construction, $\left\{e_{f(\mathcal{O})} \mid \mathcal{O} \in X / G\right\}$ is a generating set of the $\mathbb{K} G$-module $\mathbb{K} X$. It is also linearly independent over $\mathbb{K} G$, by the following reasoning. Assume that, for some coefficients $a_{\mathcal{O}}=\sum_{g \in G} \alpha_{\mathcal{O}, g} g \in \mathbb{K} G$,

$$
0=\sum_{\mathcal{O} \in X / G} e_{f(\mathcal{O})} \cdot a_{\mathcal{O}}=\sum_{\mathcal{O} \in X / G} \sum_{g \in G} \alpha_{\mathcal{O}, g} e_{f(\mathcal{O}) \cdot g}
$$

Since $G$ acts on $X$ faithfully, in the above sum each element $e_{f(\mathcal{O})}$ appears exactly once. Hence $\alpha_{\mathcal{O}, g}=0$, for all $\mathcal{O} \in X / G$ and $g \in G$. Thus we have the claim proven.

Proposition 3.4. Let $\mathcal{G}$ be a small groupoid with finite set of objects. Let $\mathcal{B}$ be the groupoid bialgebroid associated to $\mathcal{G}$. Let $M$ be an anti Yetter-Drinfel'd $\mathcal{B}$-module. Keeping the notation introduced above, there is an isomorphism of anti Yetter-Drinfel'd $\mathcal{B}$-modules

$$
M_{[l]} \cong B(s(l)) \otimes_{B_{l}} M_{l}, \quad \text { for all } l \in \mathcal{L}(\mathcal{G}) .
$$

Proof. First we construct a left $B$-module isomorphism

$$
\varphi_{l}: B(s(l)) \otimes_{B_{l}} M_{l} \rightarrow M_{[l]}, \quad g \otimes_{B_{l}} m \mapsto g \triangleright m .
$$

For any $g \in \mathcal{G}^{1}$ such that $s(g)=s(l)$, consider the map

$$
\psi_{g}: M_{g l g^{-1}} \rightarrow B(s(l)) \otimes_{B_{l}} M_{l}, \quad g \mapsto g \otimes_{B_{l}} g^{-1} \triangleright m .
$$

In order to see that the map $\psi_{g}$ does not depend on $g$, only on $g l g^{-1}$, choose another element $h \in \mathcal{G}^{1}$, such that $g l g^{-1}=h l h^{-1}$. Note that this implies in particular $t(g)=t(h)$. Then, for 
$m \in M_{g l g^{-1}}$,

$$
\begin{aligned}
\psi_{h}(m) & =h \otimes_{B_{l}} h^{-1} \triangleright m=h \otimes_{B_{l}} h^{-1} t(h) \triangleright m=h \otimes_{B_{l}} h^{-1} t(g) \triangleright m=h \otimes_{B_{l}} h^{-1} g g^{-1} \triangleright m \\
& =h h^{-1} g \otimes_{B_{l}} g^{-1} \triangleright m=t(h) g \otimes_{B_{l}} g^{-1} \triangleright m=t(g) g \otimes_{B_{l}} g^{-1} \triangleright m=g \otimes_{B_{l}} g^{-1} \triangleright m=\psi_{g}(m),
\end{aligned}
$$

where in the fifth equality we used that $h^{-1} g$ is an element of $B_{l}$. Thus we conclude by universality of a direct sum on the existence of a map $\psi: M_{[l]} \cong \oplus_{l^{\prime} \in[l]} M_{l^{\prime}} \rightarrow B(s(l)) \otimes_{B_{l}} M_{l}$, mapping $m \in M_{g l g^{-1}}$ to $\psi_{g}(m)=g \otimes_{B_{l}} g^{-1} \triangleright m$. A straightforward computation shows that $\psi$ is the inverse of $\varphi_{l}$ in (3.4).

Next we show that $B(s(l)) \otimes_{B_{l}} M_{l}$ is an anti Yetter-Drinfel'd module with respect to the direct sum decomposition $B(s(l)) \otimes_{B_{l}} M_{l} \cong \oplus_{l^{\prime} \in[l]} \psi\left(M_{l^{\prime}}\right)$, hence (3.4) is an isomorphism of anti YetterDrinfel'd modules, as stated. For $m \in M_{l}, l^{\prime} \in \mathcal{L}(\mathcal{G}), h, h^{\prime} \in \mathcal{G}^{1}$ and $y \in \mathcal{G}^{0}$, such that $h l h^{-1}=l^{\prime}$ and $s\left(h^{\prime}\right)=t(h)$,

$$
\begin{aligned}
y \triangleright\left(h \otimes_{B_{l}} m\right) & =y h \otimes_{B_{l}} m=\delta_{t(h), y} h \otimes_{B_{l}} m=\delta_{s\left(l^{\prime}\right), y} h \otimes_{B_{l}} m, \\
h^{\prime} \triangleright\left(h \otimes_{B_{l}} m\right) & =h^{\prime} h \otimes_{B_{l}} m \in \psi\left(M_{h^{\prime} l^{\prime} h^{\prime-1}}\right) .
\end{aligned}
$$

In view of Theorem 3.2 this implies that $B(s(l)) \otimes_{B_{l}} M_{l}$ is an anti Yetter-Drinfel'd module with respect to the given decomposition, hence it completes the proof.

3.B. Hochschild and cyclic homology with coefficients. In view of Proposition 2.19, there is a cyclic simplex associated to a groupoid bialgebroid $\mathcal{B}$, the right regular $\mathcal{B}$-module coring and any stable anti Yetter-Drinfel'd $\mathcal{B}$-module $M$. At degree $n$, it is given by $Z_{n}(B, M)=B^{\otimes_{R} n+1} \otimes_{B} M$ (where $B$ acts on $B^{\otimes_{R} n+1}$ via the diagonal right action). In this section we compute its Hochschild and cyclic homologies.

With an eye on the decomposition of $M$ in Section 3.A, computations start with following

Lemma 3.5. Let $\mathcal{G}$ be a small groupoid with finite set of objects. Let $\mathcal{B}$ be the groupoid bialgebroid over a field $\mathbb{K}$, associated to $\mathcal{G}$. Let $M$ be an anti Yetter-Drinfel'd $\mathcal{B}$-module. Using notations introduced in Section 3.A, there is an isomorphism of right $B_{l}$-modules

$$
B^{\otimes_{R} n+1} \otimes_{B} B(x) \cong B(x)^{\otimes_{\mathbb{K}} n+1},
$$

for all $l \in \mathcal{L}(\mathcal{G})$ and $x:=s(l)$. Here $B^{\otimes_{R} n+1} \otimes_{B} B(x)$ is understood to be a right $B_{l}$-module via the last factor and the group algebra $B_{l}$ acts on $B(x)^{\otimes_{\mathbb{K}} n+1}$ via the diagonal action.

Proof. Since $R=\mathbb{K} \mathcal{G}^{0}$ is a separable $\mathbb{K}$-algebra, $B^{\otimes_{R} n+1}$ is isomorphic to the subspace $B^{\times n+1}$ of $B^{\otimes_{\mathbb{K}} n+1}$, spanned by those elements $g_{0} \otimes_{\mathbb{K}} \ldots \otimes_{\mathbb{K}} g_{n}$ for which $s\left(g_{i}\right)=s\left(g_{i+1}\right)$, for all $i=0 \ldots n$. Thus it suffices to prove $B^{\times n+1} \otimes_{B} B(x) \cong B(x)^{\otimes_{\mathbb{K}} n+1}$. We claim that the right $B_{l}$-module map

$$
B(x)^{\otimes_{\mathbb{K}} n+1} \rightarrow B^{\times n+1} \otimes_{B} B(x), \quad g_{0} \otimes_{\mathbb{K}} \ldots \otimes_{\mathbb{K}} g_{n} \mapsto\left(g_{0} \otimes_{\mathbb{K}} \ldots \otimes_{\mathbb{K}} g_{n}\right) \otimes_{B} x
$$

is an isomorphism. Since the map

$$
B^{\times n+1} \otimes_{\mathbb{K}} B(x) \rightarrow B(x)^{\otimes_{\mathbb{K}} n+1}, \quad\left(g_{0} \otimes_{\mathbb{K}} \ldots \otimes_{\mathbb{K}} g_{n}\right) \otimes_{\mathbb{K}} h \mapsto g_{0} h \otimes_{\mathbb{K}} \ldots \otimes_{\mathbb{K}} g_{n} h
$$

factorizes through $B^{\times n+1} \otimes_{B} B(x)$, it defines a unique map

$$
B^{\times n+1} \otimes_{B} B(x) \rightarrow B(x)^{\otimes_{\mathbb{K}} n+1}, \quad\left(g_{0} \otimes_{\mathbb{K}} \ldots \otimes_{\mathbb{K}} g_{n}\right) \otimes_{B} h \mapsto g_{0} h \otimes_{\mathbb{K}} \ldots \otimes_{\mathbb{K}} g_{n} h .
$$

Obviously, (3.5) and (3.6) are mutual inverses.

Proposition 3.6. Let $\mathcal{G}$ be a small groupoid with finite set of objects. Let $\mathcal{B}$ be the groupoid bialgebroid over a field $\mathbb{K}$, associated to $\mathcal{G}$. Let $M$ be a stable anti Yetter-Drinfel'd $\mathcal{B}$-module, with decomposition (3.2). For all $l \in \mathcal{L}(\mathcal{G})$, the cyclic simplex $Z_{*}\left(B, M_{[l]}\right)=B^{\otimes_{R} *+1} \otimes_{B} M_{[l]}$ is isomorphic to $Z_{*}\left(B(s(l)), M_{l}\right)=B(s(l))^{\otimes_{\mathbb{K}} *+1} \otimes_{B_{l}} M_{l}$, corresponding (as in [JŞ, Remark 4.16]) to the module coalgebra $B(s(l))$, and stable anti Yetter-Drinfel'd module $M_{l}$, of the group bialgebra $B_{l}$. 
Proof. Combining the isomorphisms in Proposition 3.4 and Lemma 3.5, we obtain an isomorphism of vector spaces

$$
\begin{array}{ccc}
B(s(l))^{\otimes_{\mathbb{K}} n+1} \otimes_{B_{l}} M_{l} \cong & B^{\otimes_{R} n+1} \otimes_{B} B(s(l)) \otimes_{B_{l}} M_{l} \cong & B^{\otimes_{R} n+1} \otimes_{B} M_{[l]} \\
\left(g_{0} \otimes_{\mathbb{K}} \ldots \otimes_{\mathbb{K}} g_{n}\right) \otimes_{B_{l}} m & \longmapsto & \left(g_{0} \otimes_{R} \ldots \otimes_{R} g_{n}\right) \otimes_{B} m,
\end{array}
$$

with inverse $B^{\otimes_{R} n+1} \otimes_{B} M_{h l h^{-1}} \ni\left(g_{0} \otimes_{R} \ldots \otimes_{R} g_{n}\right) \otimes_{B} m \mapsto\left(g_{0} h \otimes_{\mathbb{K}} \ldots \otimes_{\mathbb{K}} g_{n} h\right) \otimes_{B_{l}} h^{-1} \triangleright m$. It is left to the reader to check that it is an isomorphism of cyclic objects.

Hochschild and cyclic homologies of a group, with coefficients in a stable anti Yetter-Drinfel'd module, were computed in [JŞ, Corollary 5.13]. Hence in what follows we relate Hochschild and cyclic homologies of the cyclic object $Z_{*}\left(B(s(l)), M_{l}\right) \cong Z_{*}\left(B, M_{[l]}\right)$ in Proposition 3.6 to the known respective homology of $Z_{*}\left(B_{l}, M_{l}\right)$.

Lemma 3.7. Let $(C, \Delta, \epsilon)$ be a coalgebra over a field $\mathbb{K}$. Consider the corresponding simplex $C^{\otimes_{\mathbb{K}}^{*+1}}$ (with face maps $\partial_{i}$ induced by $\epsilon$ and degeneracy maps $\mu_{i}$ induced by $\Delta$ ). Then the associated complex $\widetilde{C}_{*}(C)$ is acyclic, i.e. $H_{n}\left(\widetilde{C}_{*}(C)\right)=\delta_{n, 0} \mathbb{K}$, for any non-negative integer $n$. Moreover, if $(C, \Delta, \epsilon)$ is a right module coalgebra of a $\mathbb{K}$-Hopf algebra $H$ and free as a right $H$-module then $\widetilde{C}_{*}(C)$ is a free resolution of $\mathbb{K}$ in the category of right $H$-modules.

Proof. We need to show that the chain complex

$$
\cdots \stackrel{\delta_{n+1}}{\longrightarrow} C^{\otimes_{\mathbb{K}} n+1} \stackrel{\delta_{n}}{\longrightarrow} C^{\otimes_{\mathbb{K}} n} \longrightarrow \cdots \stackrel{\delta_{1}}{\longrightarrow} C \stackrel{\delta_{0}=\epsilon}{\longrightarrow} \mathbb{K} \longrightarrow 0
$$

is exact, where $\delta_{n}=\sum_{i=0}^{n}(-1)^{i} \partial_{i}$. Indeed, the map $C \otimes_{\mathbb{K}} \epsilon$ is surjective, having a section $\Delta$. Since $C$ is a faithfully flat module of the field $\mathbb{K}$, this implies surjectivity of $\epsilon$. Take an element $g \in C$ such that $\epsilon(g)=1_{\mathbb{K}}$. One easily checks that, for $z \in C^{\otimes_{\mathbb{K}} n+1}$ such that $\delta_{n}(z)=0$, $\delta_{n+1}\left(g \otimes_{\mathbb{K}} z\right)=z-\left(g \otimes_{\mathbb{K}} \delta_{n}(z)\right)=z$.

Assume now that $(C, \Delta, \epsilon)$ is a right module coalgebra of a $\mathbb{K}$-Hopf algebra $H$. Then $\widetilde{C}_{*}(C)$ is an acyclic complex in the category of right H-modules. It remains to show that $C^{\otimes_{\mathbb{K}} n+1}$ is a free $H$-module whenever $C$ is so. Indeed, in this case $C^{\otimes_{\mathbb{K}} n+1}$ is free as a right module of $H^{\otimes_{\mathbb{K}} n+1}$ via factorwise action. By [JŞ, Lemma 2.10] (cf. Lemma 2.25), $H^{\otimes_{\mathbb{K}} n+1}$ is free as a right $H$-module via the diagonal action. Hence $C^{\otimes_{\mathbb{K}} n+1} \cong C^{\otimes_{\mathbb{K}} n+1} \otimes_{H^{\otimes_{\mathbb{K}} n+1}} H^{\otimes_{\mathbb{K}} n+1}$ is a free right $H$-module.

Lemma 3.8. Let $H$ be a Hopf algebra over a field $\mathbb{K}$ and let $i: C \rightarrow C^{\prime}$ be a morphism of right $H$-module coalgebras. Let $M$ be a stable anti Yetter-Drinfel'd $H$-module. If both $C$ and $C^{\prime}$ are free as right $H$-modules, then the induced morphism $i_{*}: Z_{*}(C, M) \rightarrow Z_{*}\left(C^{\prime}, M\right)$ of cyclic objects gives rise to isomorphisms both of Hochschild and cyclic homologies. That is,

$$
\mathrm{HH}_{*}(C, M) \cong \mathrm{HH}_{*}\left(C^{\prime}, M\right) \quad \text { and } \quad \mathrm{HC}_{*}(C, M) \cong \mathrm{HC}_{*}\left(C^{\prime}, M\right) .
$$

Proof. By Lemma 3.7, both $\widetilde{C}_{*}(C)$ and $\widetilde{C}_{*}\left(C^{\prime}\right)$ are free resolutions of $\mathbb{K}$ over $H$. Thus $i^{\otimes_{\mathbb{K}} *+1}$ : $\widetilde{C}_{*}(C) \rightarrow \widetilde{C}_{*}\left(C^{\prime}\right)$ is a quasi-isomorphism. Then so is $i^{\otimes_{\mathbb{K}} *+1} \otimes_{H} M: \widetilde{C}_{*}(C) \otimes_{H} M \rightarrow \widetilde{C}_{*}\left(C^{\prime}\right) \otimes_{H} M$, yielding an isomorphism

$$
\mathrm{HH}_{n}(C, M) \equiv \mathrm{H}_{n}\left(\widetilde{C}_{*}(C) \otimes_{H} M\right) \cong \mathrm{H}_{n}\left(\widetilde{C}_{*}\left(C^{\prime}\right) \otimes_{H} M\right) \equiv \mathrm{HH}_{n}\left(C^{\prime}, M\right),
$$

for all non-negative integer $n$. The isomorphism of Hochschild homologies implies the isomorphism of cyclic homologies, see e.g. (a dual form of) [Lo, 2.2.3].

TheOREM 3.9. Let $\mathcal{G}$ be a small groupoid with finite set of objects. Let $\mathcal{B}$ be the groupoid bialgebroid over a field $\mathbb{K}$, associated to $\mathcal{G}$. Let $M$ be a stable anti Yetter-Drinfel'd $\mathcal{B}$-module, with decomposition (3.2). For all $l \in \mathcal{L}(\mathcal{G})$,

$$
\mathrm{HH}_{*}\left(B, M_{[l]}\right) \cong \mathrm{HH}_{*}\left(B_{l}, M_{l}\right) \quad \text { and } \quad \mathrm{HC}_{*}\left(B, M_{[l]}\right) \cong \mathrm{HC}_{*}\left(B_{l}, M_{l}\right)
$$

Proof. By Proposition 3.6, the cyclic objects $Z_{*}\left(B, M_{[l]}\right)$ and $Z_{*}\left(B(s(l)), M_{l}\right)$ are isomorphic. Hence $\mathrm{HH}_{*}\left(B, M_{[l]}\right) \cong \mathrm{HH}_{*}\left(B(s(l)), M_{l}\right)$. Furthermore, the right $B_{l}$-module coalgebra $B(s(l))$ is a free right $B_{l}$-module by Lemma 3.3. Hence we conclude by Lemma 3.8 that the inclusion map $B_{l} \hookrightarrow B(s(l))$ induces an isomorphism $\mathrm{HH}_{*}\left(B(s(l)), M_{l}\right) \cong \mathrm{HH}_{*}\left(B_{l}, M_{l}\right)$. Combination of these isomorphisms proves the theorem. 
Let us assume now that $\mathbb{K}$ is a field of characteristic zero. In view of Theorem 3.9, one can compute also

$$
\mathrm{HH}_{*}(B, M) \cong \bigoplus_{[l] \in \mathcal{T}(\mathcal{G})} \operatorname{HH}_{*}\left(B_{l}, M_{l}\right) \quad \text { and } \quad \operatorname{HC}_{*}(B, M) \cong \bigoplus_{[l] \in \mathcal{T}(\mathcal{G})} \operatorname{HC}_{*}\left(B_{l}, M_{l}\right)
$$

Since $B_{l}$ is a group algebra of $\mathcal{G}_{l}^{1}$ (cf. (3.3)), $\operatorname{HH}_{*}\left(B_{l}, M_{l}\right)=H_{*}\left(\mathcal{G}_{l}^{1}, M_{l}\right)$ is the group homology of $\mathcal{G}_{l}^{1}$ with coefficients in $M_{l}$. Applying [JS, Corollary 5.13], for the cyclic homology we get

$$
\operatorname{HC}_{*}\left(B_{l}, M_{l}\right)= \begin{cases}\bigoplus_{i>0} \mathrm{H}_{*-2 i}\left(\mathcal{G}_{l}^{1} /\langle l\rangle, M_{l}\right), & \text { if } l \text { has finite order } \\ \mathrm{H}_{*}\left(\mathcal{G}_{l}^{1} /\langle l\rangle, M_{l}\right), & \text { if } l \text { has infinite order. }\end{cases}
$$

3.C. Cyclic homology of groupoids. The results in Section 3.B can be specialized further to stable anti Yetter-Drinfel'd modules provided by groupoid Galois extensions, cf. Proposition 2.23. This enables us, in particular, to compute ordinary (i.e. non-relative) Hochschild and cyclic homologies of a groupoid.

Let $\mathcal{G}$ be a small groupoid with finite set of objects. Let $\mathcal{B}$ be the groupoid bialgebroid over a field $\mathbb{K}$, associated to $\mathcal{G}$. Recall from [CaDGr, Section 3] that a Galois extension $S \subseteq T$ by $\mathcal{B}$ has an equivalent description as follows. $T$ is a strongly $\mathcal{G}$-graded $\mathbb{K}$-algebra, that is, $T \cong \oplus_{g \in \mathcal{G}^{1}} T_{g}$ (as a vector space),

$$
T_{g} T_{g^{\prime}}= \begin{cases}T_{g \circ g^{\prime}} & \text { if } s(g)=t\left(g^{\prime}\right) \\ 0 & \text { if } s(g) \neq t\left(g^{\prime}\right),\end{cases}
$$

$1_{T}=\sum_{x \in \mathcal{G}^{0}} 1_{T_{x}}$, and $S$ is equal to the subalgebra $\oplus_{x \in \mathcal{G}^{0}} T_{x}$. Note that each direct summand $T_{g}$ is an $R=\mathbb{K} \mathcal{G}^{0}$-module via $v \cdot x:=v 1_{T_{x}}=\delta_{s(g), x} v$, for $v \in T_{g}$ and $x \in \mathcal{G}^{0}$.

By Proposition 2.23, $T_{S}:=S \widehat{\otimes}_{S} T$ is a stable anti Yetter-Drinfel'd module. In the following lemma its structure is investigated.

Lemma 3.10. Let $\mathcal{G}$ be a small groupoid with finite set of objects. Let $\mathcal{B}$ be the groupoid bialgebroid over a field $\mathbb{K}$, associated to $\mathcal{G}$. Let $S \subseteq T$ be a Galois extension by $\mathcal{B}$. Using the notations introduced in Section 3.A, there is an isomorphism of anti Yetter-Drinfel'd modules

$$
T_{S} \cong \bigoplus_{l \in \mathcal{L}(\mathcal{G})} S \widehat{\otimes}_{S} T_{l}
$$

The (Miyashita-Ulbrich) action is given, for $g \in \mathcal{G}^{1}, l \in \mathcal{L}(\mathcal{G})$ and $v \in T_{l}$, by

$$
g \triangleright p_{T_{l}}(v)=\sum_{i=1}^{n} p_{T_{l}}\left(b_{i} v a_{i}\right) \text {, }
$$

where $p_{T_{l}}: T_{l} \rightarrow S \widehat{\otimes}_{S} T_{l}$ denotes the canonical epimorphism and the elements $\left\{a_{1}, \ldots, a_{n}\right\} \subseteq T_{g^{-1}}$ and $\left\{b_{1}, \ldots, b_{n}\right\} \subseteq T_{g}$ satisfy $\sum_{i=1}^{n} a_{i} b_{i}=1_{T_{s(g)}}$.

Proof. Since $T \cong \oplus_{g \in \mathcal{G}^{1}} T_{g}$ is $\mathcal{G}$-graded, each direct summand $T_{g}$ is an $S$-bimodule. Hence

$$
S \widehat{\otimes}_{S} T \cong \bigoplus_{g \in \mathcal{G}^{1}} S \widehat{\otimes}_{S} T_{g}
$$

We claim that only those elements $g \in \mathcal{G}^{1}$ give non-zero contribution to this direct sum, for which $s(g)=t(g)$. Recall that $S \widehat{\otimes}_{S} T_{g}$ is isomorphic to the quotient of $T_{g}$ with respect to the commutator subspace $\left[S, T_{g}\right]=\left\{q v-v q \mid q \in S, v \in T_{g}\right\}$. Take an element $g \in \mathcal{G}^{1}$ such that $s(g) \neq t(g)$ and an element $v \in T_{g}$. By strong grading of $T$, there exist elements $\left\{a_{1}, \ldots, a_{n}\right\} \subseteq T_{g^{-1}}$ and $\left\{b_{1}, \ldots, b_{n}\right\} \subseteq T_{g}$ such that $\sum_{i=1}^{n} a_{i} b_{i}=1_{T_{s(g)}}$. Then

$$
\sum_{i=1}^{n}\left[v a_{i}, b_{i}\right]=\sum_{i=1}^{n} v a_{i} b_{i}-b_{i} v a_{i}=v 1_{T_{s(g)}}=v,
$$

where the penultimate equality follows by the fact that, for all values of $i, b_{i} v a_{i} \in T_{g} T_{g} T_{g^{-1}}$ is zero by the assumption that $s(g) \neq t(g)$. Since for all values of $i, v a_{i} \in T_{g^{-1}} T_{g}=T_{s(g)} \subseteq S$, we conclude that $T_{g} \subseteq\left[S, T_{g}\right]$. Since the converse inclusion is obvious, we have $S \widehat{\otimes}_{S} T_{g} \cong T_{g} /\left[S, T_{g}\right]=0$ proven.

In order to write down the Miyashita-Ulbrich action, consider again $g \in \mathcal{G}^{1}$ and elements $\left\{a_{1}, \ldots, a_{n}\right\} \subseteq T_{g^{-1}}$ and $\left\{b_{1}, \ldots, b_{n}\right\} \subseteq T_{g}$ such that $\sum_{i=1}^{n} a_{i} b_{i}=1_{T_{s(g)}}$. The canonical map can : $T \otimes_{S} T \rightarrow T \otimes_{R} B$ satisfies

$$
\operatorname{can}\left(\sum_{i=1}^{n} a_{i} \otimes_{S} b_{i}\right)=\sum_{i=1}^{n} a_{i} b_{i} \otimes_{R} g=1_{T_{s(g)}} \otimes_{R} g=1_{T} \cdot s(g) \otimes_{R} g=1_{T} \otimes_{R} g .
$$


Hence the stated form of the Miyashita-Ulbrich action follows by (2.31).

Corollary 3.11. Let $\mathcal{G}$ be a small groupoid with finite set of objects. Let $\mathcal{B}$ be the groupoid bialgebroid over a field $\mathbb{K}$, associated to $\mathcal{G}$. Let $S \subseteq T$ be a Galois extension by $\mathcal{B}$. Using the notations introduced in Section 3.A, the S-relative Hochschild and cyclic homologies of $T$ are given, respectively, by

$$
\mathrm{HH}_{*}(T / S)=\bigoplus_{[l] \in \mathcal{T}(\mathcal{G})} \mathrm{HH}_{*}\left(B_{l}, S \widehat{\otimes}_{S} T_{l}\right) \quad \text { and } \quad \mathrm{HC}_{*}(T / S)=\bigoplus_{[l] \in \mathcal{T}(\mathcal{G})} \mathrm{HC}_{*}\left(B_{l}, S \widehat{\otimes}_{S} T_{l}\right)
$$

Proof. By Theorem 2.26, $\mathrm{HH}_{*}(T / S) \cong \mathrm{HH}_{*}\left(B, T_{S}\right)$ and $\mathrm{HC}_{*}(T / S) \cong \mathrm{HC}_{*}\left(B, T_{S}\right)$. Hence the claim follows by Theorem 3.9 and considerations following it, together with Lemma 3.10.

A particular example $R \subseteq B$ of a Galois extension by a groupoid $\mathcal{G}$ is provided by the inclusion of the base algebra $R=\mathbb{K} \mathcal{G}^{0}$ in the groupoid algebra $B=\mathbb{K} \mathcal{G}^{1}$. Applying Corollary 3.11 to it, we obtain formulae for the $R$-relative Hochschild and cyclic homologies of the groupoid $\mathcal{G}$. Since the base algebra $R=\mathbb{K} \mathcal{G}^{0}$ is separable over $\mathbb{K}$, relative homologies coincide with ordinary ones, cf. [Kad]. Therefore we obtain the following corollary, extending results in [Burg] on cyclic homology of groups. Similar expressions were derived also by Crainic in [Cra] for étale groupoids.

COROLlary 3.12. Let $B$ be the groupoid algebra of a small groupoid $\mathcal{G}$ with finite set of objects over a field $\mathbb{K}$ of characteristic zero. Using the notations introduced in Section 3.A we have

$$
\begin{aligned}
\mathrm{HH}_{*}(B)= & \bigoplus \bigoplus_{[l] \in \mathcal{T}(\mathcal{G})} \mathrm{H}_{*}\left(\mathcal{G}_{l}^{1}, \mathbb{K}\right), \\
\mathrm{HC}_{*}(B)= & \left(\bigoplus_{\substack{[l] \in \mathcal{T}(\mathcal{G}) \\
\operatorname{ord}(l)<\infty}} \bigoplus_{i \geq 0} \mathrm{H}_{*-2 i}\left(\mathcal{G}_{l}^{1} /\langle l\rangle, \mathbb{K}\right)\right) \bigoplus\left(\bigoplus_{\substack{[l] \in \mathcal{T}(\mathcal{G}) \\
\operatorname{ord}(l)=\infty}} \mathrm{H}_{*}\left(\mathcal{G}_{l}^{1} /\langle l\rangle, \mathbb{K}\right)\right)
\end{aligned}
$$

Recall that a groupoid $\mathcal{G}$ is connected if, for any $x, y \in \mathcal{G}^{0}$, there exists at least one $g \in \mathcal{G}^{1}$ such that $s(g)=x$ and $t(g)=y$. For a connected groupoid $\mathcal{G}$ we fix an object $x \in \mathcal{G}^{0}$ and we denote by $G$ the group of loops $l \in \mathcal{L}(\mathcal{G})$ such that $s(l)=t(l)=x$. (Since $\mathcal{G}$ is connected by assumption, different choices of $x$ lead to isomorphic subgroups $G$ of $\mathcal{G}$.) Let $\mathcal{T}(G)$ denote the set of conjugacy classes in $G$ and let $\left\{g_{\sigma} \mid \sigma \in \mathcal{T}(G)\right\}$ be a transversal of $\mathcal{T}(G)$.

LEMMA 3.13. Let $\mathcal{G}$ be a connected groupoid. Keeping the above notation, there is an one-to-one correspondence between $\mathcal{T}(\mathcal{G})$ and $\mathcal{T}(G)$.

Proof. We have to show that any orbit in $\mathcal{L}(\mathcal{G})$ for the adjoint action contains precisely one element of the set $\left\{g_{\sigma} \mid \sigma \in \mathcal{T}(G)\right\}$. That is, any loop $l$ in $\mathcal{G}$ is equivalent to a certain $g_{\sigma}$ and that two elements $g_{\sigma}$ and $g_{\tau}$ are equivalent if, and only if $\sigma=\tau$. First, let us take $l \in \mathcal{L}(\mathcal{G})$. Since $\mathcal{G}$ is connected, there is a morphism $g$ such that $s(g)=x$ and $t(g)=s(l)$. Let $l^{\prime}:=g^{-1} \circ l \circ g$. By construction, $l^{\prime} \in G$, so there are $h \in G$ and $\sigma \in \mathcal{T}(G)$ such that $h \circ l^{\prime} \circ h^{-1}=g_{\sigma}$. Since $l=\left(g \circ h^{-1}\right) \circ g_{\sigma} \circ\left(g \circ h^{-1}\right)^{-1}$ it follows that $l$ and $g_{\sigma}$ are conjugated in $\mathcal{L}(\mathcal{G})$.

Let us take $\sigma$ and $\tau$ in $\mathcal{T}(G)$ and assume that $g_{\sigma}$ and $g_{\tau}$ define the same element in $\mathcal{T}(\mathcal{G})$. Then there is $g \in \mathcal{G}^{1}$ such that $g_{\sigma}=g \circ g_{\tau} \circ g^{-1}$. Since both loops $g_{\sigma}$ and $g_{\tau}$ have the same source, we deduce that the source and the target of $g$ must be $x$. Hence $g \in G$ and the conjugacy classes of $g_{\sigma}$ and $g_{\tau}$ in $\mathcal{T}(G)$ are equal. In conclusion, $\sigma=\tau$.

REMARK 3.14. Lemma 3.13 tells us, in particular, that $\left\{g_{\sigma} \mid \sigma \in \mathcal{T}(G)\right\}$ is also a transversal of $\mathcal{T}(\mathcal{G})$. Explicitly, a given element $g_{\sigma}$ represents the following orbit $\widetilde{\sigma} \in \mathcal{T}(\mathcal{G})$. Recall that we defined the group $G$ in terms of a fixed object $x$ in $\mathcal{G}^{0}$. For every $y \in \mathcal{G}^{0}$ we pick up a fixed $g_{y} \in \operatorname{Hom}_{\mathcal{G}}(x, y)$. It defines a group isomorphism $G \rightarrow \operatorname{Hom}_{\mathcal{G}}(y, y), h \mapsto g_{y} \circ h \circ g_{y}^{-1}$. It maps $\sigma \in \mathcal{T}(G)$ to the conjugacy class $\sigma_{y}=\left\{g_{y} \circ h \circ g_{y}^{-1} \mid h \in \sigma\right\}$ in the group Hom $\operatorname{G}_{\mathcal{G}}(y, y)$. The orbit of $g_{\sigma}$ for the adjoint $\mathcal{G}$-action is $\widetilde{\sigma}=\bigcup_{y \in \mathcal{G}^{0}} \sigma_{y}$. 
COROLLARY 3.15. Let $\mathcal{G}$ be a connected groupoid with finitely many objects. Let $M$ be a stable anti Yetter-Drinfel'd module over B, where $B$ is the groupoid algebra of $\mathcal{G}$ over a field of characteristic zero.

(i) $M_{G}:=\bigoplus_{g \in G} M_{g}$ is a stable anti Yetter-Drinfel'd module over $\mathbb{K} G$ and the inclusions $\mathbb{K} G \subseteq$ $B$ and $M_{G} \subseteq M$ induce isomorphisms

$$
\mathrm{HH}_{*}(B, M) \cong \mathrm{HH}_{*}\left(\mathbb{K} G, M_{G}\right) \quad \text { and } \quad \mathrm{HC}_{*}(B, M) \cong \mathrm{HC}_{*}\left(\mathbb{K} G, M_{G}\right) .
$$

(ii) The inclusion $\mathbb{K} G \subseteq B$ induces isomorphisms

$$
\mathrm{HH}_{*}(B) \cong \mathrm{HH}_{*}(\mathbb{K} G) \quad \text { and } \quad \mathrm{HC}_{*}(B) \cong \mathrm{HC}_{*}(\mathbb{K} G) .
$$

Proof. (i) Obviously $M_{G}$ is a stable anti Yetter-Drinfel'd module over $\mathbb{K} G$. Consider the following diagram.

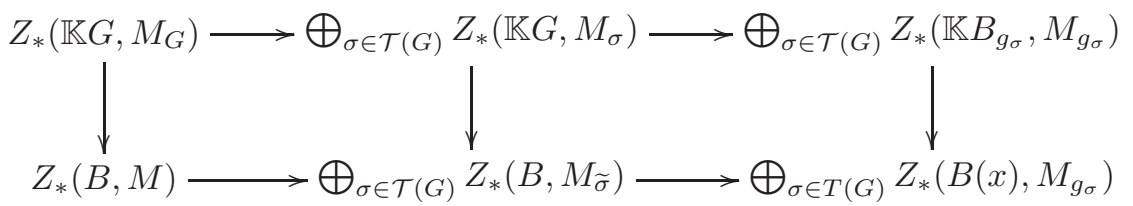

The leftmost morphism in the bottom row comes from the decomposition (3.2), while the other one is the direct sum of the arrows that were constructed in Proposition 3.6. Note that both maps are induced by appropriate inclusions and they are isomorphisms. The morphisms in the top row have the same properties, as any group can be regarded as a groupoid with one object. By definition, the vertical arrows are the canonical morphisms induced by inclusions, so they make the squares commutative. In view of the proof of Theorem 3.9, the rightmost vertical arrow gives isomorphisms both of Hochschild and cyclic homologies. Then also the leftmost vertical morphism does so.

(ii) The subalgebra $R:=\mathbb{K} X$ of $B$ is a separable $\mathbb{K}$-algebra. Hence $\operatorname{HH}_{*}(B) \cong \operatorname{HH}_{*}(B, R)$. By Lemma 3.10 we know that $M:=R \widehat{\otimes}_{R} B$ is a stable anti Yetter-Drinfel'd module over $B$. Since for any $g \in \mathcal{G}$, either $[R, \mathbb{K} g]=\mathbb{K} g$ or $[R, \mathbb{K} g]=0$, depending on the fact that $g$ is a loop or not, we get $M:=\bigoplus_{g \in \mathcal{L}(\mathcal{G})} \mathbb{K} g$. Hence $M_{G}:=\mathbb{K} G$. Obviously the action of $B$ on $\mathbb{K} G$ induced from the Ulbrich-Miyashita action is the adjoint action of $\mathbb{K} G$ on itself. We conclude by applying the first part of the corollary and the isomorphisms $\mathrm{HH}_{*}(\mathbb{K} G) \cong \mathrm{HH}_{*}\left(\mathbb{K} G,(\mathbb{K} G)_{a d}\right)$ and $\mathrm{HC}_{*}(\mathbb{K} G) \cong \mathrm{HC}_{*}\left(\mathbb{K} G,(\mathbb{K} G)_{a d}\right)$, cf. [JS].

Our final aim is to compute $\mathrm{HC}_{*}(B)$, the ordinary cyclic homology of the groupoid algebra $B$ of a groupoid $\mathcal{G}$ that may have infinite number of objects. For such a groupoid, its groupoid algebra $B$ is not unital anymore. Nevertheless, to define cyclic homology of $B$ one can proceed as for unital algebras, cf. [Lo, Chapter 2, §2.1]. The point is that Connes' complex $C^{\lambda}(B)$ still exists, although it is now associated to a precyclic object, that is to a presimplicial structure endowed with cyclic operators. Here, by presimplicial object we mean a sequence of objects together only with face maps. The defining properties of face maps and cyclic operators are the same as in the definition of cyclic objects, neglecting of course the relations that involve the degeneracy maps. The key ingredient of our computation is a description of any groupoid as a direct limit of groupoids with finitely many objects. Since cyclic homology is defined as homology of Connes' complex and the homology functor commutes with direct limits, we obtain cyclic homology of an arbitrary groupoid as a direct limit. Note however that, for non-unital algebras, Hochschild homology is constructed in a different way. For the definition, see for example [Lo, Chapter 1, §1.2]. Therefore, we can not apply the same arguments to compute Hochschild homology of an arbitrary groupoid.

THEOREM 3.16. Let $\mathcal{G}$ be a connected groupoid. If $B$ is the groupoid algebra over a field $\mathbb{K}$ of characteristic zero, then

$$
\mathrm{HC}_{*}(B) \cong \mathrm{HC}_{*}(\mathbb{K} G) .
$$

Proof. Let $x$ be a given object in $\mathcal{G}^{0}$. Let $G$ denote the group of loops $l \in \mathcal{L}(\mathcal{G})$ such that $s(l)=x$. We order the set

$$
\mathfrak{X}:=\left\{X \subset \mathcal{G}^{0} \mid x \in X \text { and } X \text { is finite }\right\}
$$


with respect to inclusion. Trivially $\mathfrak{X}$ is a direct system. For $X \in \mathfrak{X}$ we define $\mathcal{G}_{X}$ to be the full subgroupoid of $\mathcal{G}$ such that $\mathcal{G}_{X}^{0}=X$ and we denote its groupoid algebra by $B_{X}$. Note that $B=\bigcup_{X \in \mathfrak{X}} B_{X}$, so $C_{*}^{\lambda}(B)=\bigcup_{X \in \mathfrak{X}} C_{*}^{\lambda}\left(B_{X}\right)$. As the direct limit in the category of vector spaces is exact, it follows that the homology functor and direct limit commute. Thus

$$
\mathrm{HC}_{*}(B) \cong \mathrm{H}_{*}\left(C_{*}^{\lambda}(B)\right) \cong \mathrm{H}_{*}\left(\lim _{\bar{X} \in \mathfrak{X}} C_{*}^{\lambda}\left(B_{X}\right)\right) \cong \lim _{\bar{X} \in \mathfrak{X}} \mathrm{H}_{*}\left(C_{*}^{\lambda}\left(B_{X}\right)\right) \cong \lim _{\bar{X} \in \mathfrak{X}} \mathrm{HC}_{*}\left(B_{X}\right),
$$

where the latter direct system is defined by the canonical maps $\operatorname{HC}_{*}\left(B_{X}\right) \rightarrow \mathrm{HC}_{*}\left(B_{Y}\right)$, with $X$, $Y$ in $\mathfrak{X}$ such that $X \subset Y$. We claim that these maps are isomorphisms. Indeed, let us consider the following commutative diagram

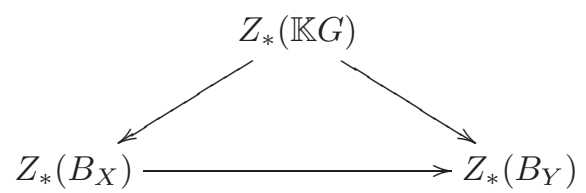

By the second part of Corollary 3.15, the oblique arrows induce isomorphisms in cyclic homology. Then, passing to cyclic homology, also the horizontal map yields an isomorphism. We can now conclude the proof of the theorem by remarking that, for any $X \in \mathfrak{X}$,

$$
\mathrm{HC}_{*}\left(B_{X}\right) \cong \lim _{\bar{X} \in \mathfrak{X}} \mathrm{HC}_{*}\left(B_{X}\right) .
$$

Thus, taking $X=\{x\}$ we get the required isomorphism.

The computation performed in Theorem 3.16 can be extended to an arbitrary (discrete) groupoid $\mathcal{G}$. Let $\left(\mathcal{G}_{i}\right)_{i \in I}$ be the connected components of $\mathcal{G}$. For each $i$ we pick up $x_{i} \in \mathcal{G}_{i}^{0}$ and we denote the set of loops $l$ with $s(l)=x_{i}$ by $G_{i}$. We have the following result.

COROLlaRY 3.17. Let $\mathcal{G}$ be a discrete groupoid. If B denotes the groupoid algebra of $\mathcal{G}$ over a field of characteristic zero, then

$$
\mathrm{HC}_{*}(B) \cong \bigoplus_{i \in I} \mathrm{HC}_{*}\left(\mathbb{K} G_{i}\right) .
$$

In addition, if $\mathcal{G}_{i}^{0}$ is a finite set for every $i \in I$, then a similar isomorphism holds in Hochschild homology.

Proof. For $i \in I$, let $B_{i}$ be the groupoid algebra of $\mathcal{G}_{i}$. As a vector space, $B$ is isomorphic to $\bigoplus_{i \in I} B_{i}$. Via this identification, the multiplication of $B$ can be extended to the direct sum. It is easy too see that, for two families $\left(b_{i}^{\prime}\right)_{i \in I}$ and $\left(b_{i}^{\prime \prime}\right)_{i \in I}$ in $\bigoplus_{i \in I} B_{i}$, we have

$$
\left(b_{i}^{\prime}\right)_{i \in I} \cdot\left(b_{i}^{\prime \prime}\right)_{i \in I}=\left(b_{i}^{\prime} b_{i}^{\prime \prime}\right)_{i \in I} .
$$

The corollary now follows by the isomorphism $\mathrm{HC}_{*}\left(\bigoplus_{i \in I} B_{i}\right) \cong \bigoplus_{i \in I} \mathrm{HC}_{*}\left(B_{i}\right)$. If $I$ is a finite set, then this isomorphism can be found in [Lo, Exercise 2.2.1]. Since cyclic homology and direct limits commute, the isomorphism can be extended for an arbitrary set $I$.

Since for non-unital algebras Hochschild homology is constructed in a different way (cf. [Lo, Chapter $1, \S 1.2]$ ), the above arguments can not be applied to deduce an isomorphism for Hochschild homology, analogous to (3.7). Nevertheless, in the case when $\mathcal{G}^{0}$ is finite, $B$ is an unital algebra. Thus $\mathrm{HH}_{*}(B)$ can be computed as the Hochschild homology of Connes' cyclic object. The required isomorphism now follows by [We, Theorem 9.1.8], proceeding as for cyclic homology.

As an application of Corollary 3.17 we shall compute $\mathrm{HH}_{*}(B)$ and $\mathrm{HC}_{*}(B)$, where $B$ is the groupoid algebra of the groupoid associated to a $G$-set $X$.

Throughout the remaining part of the paper we fix an arbitrary (discrete) group $G$ that acts to the left on an arbitrary set $X$. The action of $G$ on $X$ maps a pair $(x, g) \in X \times G$ to ${ }^{g} x \in X$. For a $G$-set $X$ as above, one construct a groupoid $\mathcal{G}$ as follows. By definition, the set of objects in $\mathcal{G}$ is $\mathcal{G}^{0}:=X$ while, for $x, y \in X$, we set

$$
\operatorname{Hom}_{\mathcal{G}}(x, y)=\left\{(x, g) \in X \times G \mid{ }^{g} x=y\right\} .
$$


Note that the source of $(x, g)$ is $x$ and its target is ${ }^{g} x$. Thus the composition $(x, g) \circ\left(x^{\prime}, g^{\prime}\right)$ is defined if, and only if $x=g^{\prime} x^{\prime}$ and, in this case

$$
(x, g) \circ\left(x^{\prime}, g^{\prime}\right):=\left(x^{\prime}, g g^{\prime}\right) .
$$

The set of morphisms in $\mathcal{G}$ is $\mathcal{G}^{1}=X \times G$. Therefore, the groupoid algebra $B$ of $\mathcal{G}$ has $X \times G$ as a basis. To describe the multiplication on this basis let us recall some well-known facts about twisted semigroup algebras by a 2-cocycle. Let $S$ be a semigroup. A function $\omega: S \times S \rightarrow \mathbb{K}$ is called a 2-cocycle if, for any $p_{1}, p_{2}, p_{3}$ in $S$

$$
\omega\left(p_{1}, p_{2}\right) \omega\left(p_{1} p_{2}, p_{3}\right)=\omega\left(p_{2}, p_{3}\right) \omega\left(p_{1}, p_{2} p_{3}\right) .
$$

The semigroup algebra of $S$ is defined as in the group case: as a vector space it has $S$ as a basis and the multiplication on this basis is given by the multiplication in $S$. We shall denote this algebra by $\mathbb{K} S$. The cocycle $\omega$ can be used to deform the multiplication of $\mathbb{K} S$ such that we get another associative algebra structure on the vector space $\mathbb{K} S$. Its multiplication is defined by

$$
p_{1} \cdot p_{2}=\omega\left(p_{1}, p_{2}\right) p_{1} p_{2}
$$

The resulting algebra will be denoted by $\mathbb{K}_{\omega} S$. Certainly, it is not unital in general. Still, even if $S$ has no neutral element, the algebra $\mathbb{K}_{\omega} S$ may have a unit $e:=\sum_{x \in X} x$, where $X$ is an appropriate finite subset of $S$. In fact, it is easy to see that $e$ is a unit element in the algebra $\mathbb{K}_{\omega}$ if, and only if $\omega$ is $X$-normalized, i.e. for any $p, q \in S$

$$
\sum_{\{x \in X \mid x p=t\}} \omega(x, p)=\delta_{p, q}=\sum_{\{x \in X \mid p x=q\}} \omega(p, x) .
$$

Let us turn back to the groupoid algebra of $\mathcal{G}$, where $X$ is a $G$-set. We define the semigroup $S:=X \times G$ with the multiplication

$$
(x, g)(y, h)=(y, g h) .
$$

It is not difficult to see that $\omega_{X}: S \times S \rightarrow \mathbb{K}$, given by

$$
\omega_{X}((x, g),(y, h)):= \begin{cases}0, & \text { if }{ }^{h} y \neq x \\ 1, & \text { if }{ }^{h} y=x\end{cases}
$$

is a 2-cocycle, which is $X$-normalized if, and only if $X$ is finite. Obviously, $B=\mathbb{K}_{\omega} S$ as non-unital algebras, in general. For a finite $G$-set $X$, this is an equality of unital algebras.

We denote the set of $G$-orbits in $X$ by $\mathfrak{X}$. Let us remark that there is an one-to-one correspondence between $\mathfrak{X}$ and the set of connected components of $\mathcal{G}$. This bijection maps an orbit $\mathfrak{o} \in \mathfrak{X}$ to the full subgroupoid $\mathcal{G}_{\mathfrak{o}}$ defined uniquely such that $\mathcal{G}_{\mathfrak{o}}^{0}=\mathfrak{o}$. We choose a transversal $\left\{x_{\mathfrak{o}} \in X \mid \mathfrak{o} \in \mathfrak{X}\right\}$ for $\mathfrak{X}$. Thus, any $x \in X$ is in the orbit of a certain $x_{\mathfrak{o}}$ and $x_{\mathfrak{o}^{\prime}}$ and $x_{\mathfrak{o}^{\prime \prime}}$ are in the same orbit if, and only if $\mathfrak{o}^{\prime}=\mathfrak{o}^{\prime \prime}$. Moreover, the set $G_{\mathfrak{o}}$ of loops $l$ such that $s(l)=x_{\mathfrak{o}}$ is the stabilizer of $x_{\mathfrak{o}}$

$$
G_{\mathfrak{o}}:=\left\{g \in G \mid{ }^{g} x_{\mathfrak{o}}=x_{\mathfrak{o}}\right\} .
$$

Hence, a direct application of Corollary 3.17 yields the following.

Theorem 3.18. Let $X$ be a G-set. If $\omega_{X}$ denotes the 2-cocycle in (3.8) and $\mathbb{K}$ is a field of characteristic zero, then

If in addition $X$ is finite, then

$$
\mathrm{HC}_{*}\left(\mathbb{K}_{\omega_{X}} G\right)=\bigoplus_{\mathfrak{o} \in \mathfrak{X}} \mathrm{HC}_{*}\left(\mathbb{K} G_{\mathfrak{o}}\right) .
$$

$$
\mathrm{HH}_{*}\left(\mathbb{K}_{\omega_{X}} G\right)=\bigoplus_{\mathfrak{o} \in \mathfrak{X}} \mathrm{HH}_{*}\left(\mathbb{K} G_{\mathfrak{o}}\right)
$$

\section{ACKNOWLEDGMENTS}

The first author was financially supported by the Hungarian Scientific Research Fund OTKA K 68195 and the Bolyai János Scholarship, while the second author was supported by Contract 2-CEx06-11-20 of the Romanian Ministry of Education and Research. Both authors are grateful to Bachuki Mesablishvili, Zoran Škoda and the referee for their helpful comments. 


\section{REFERENCES}

[AM] S.E. Akrami and S. Majid, Braided cyclic cocycles and non-associative geometry, J. Math. Phys. 45 (2004), 3883-3911.

[Be] J. Beck, Distributive laws, Lecture Notes in Mathematics 80, 119-140, Springer Verlag, 1969.

[BB] G. Böhm and T. Brzeziński, Strong connections and the relative Chern-Galois character for corings Int. Math. Res. Not. 2005, no. 42, 2579-2625.

[BCM] T. Brzeziński, S. Caenepeel and G. Militaru, Doi-Koppinen modules for quantum groupoids, J. Pure Appl. Algebra 175 (2002), 45-62.

[BMa] T. Brzeziński and S. Majid, Coalgebra bundles, Commun. Math. Phys. 191 (1998), 467492.

[BMi] T. Brzeziński and G. Militaru, Bialgebroids, $\times_{A}$-bialgebras and duality, J. Algebra 251 (2002), $279-294$.

[Burg] D. Burghelea, The cyclic homology of the group rings, Comment. Math. Helv. 60 (1985), 354-365.

[Burr] E. Burroni, Algébres non déterministiques et D-catégories, Cahiers de Topologie et Géometrie Différentielle 14 (1973), 417-475, 480-481.

[CaDGr] S. Caenepeel and E. De Groot, Galois theory for weak Hopf algebras, to appear in Rev. Roumaine Math. Pures Appl., preprint arXiv:math/0406186.

[CM95] A. Connes and H. Moscovici, Local index formula in noncommutative geometry, Geom. Funct. Anal. 5 (1995), 174-243.

[CM98] Connes and H. Moscovici, Hopf algebras, cyclic cohomology and the transverse index theorem, Comm. Math. Phys. 198 (1998), 199-246.

[CM01] Connes and H. Moscovici, Differential cyclic cohomology and Hopf algebraic structures in transverse geometry, in: Essays on geometry and related topics. Vol 1-2, pp 217-255, Monogr. Enseign. Math. 38, Enseignement Math., Geneva, 2001.

[Cra] M. Crainic, Cyclic cohomology of étale groupoids: the general case, K-Theory 17 (1999), 319-362.

[EM] S. Eilenberg and J. C. Moore, Adjoint functors and triples, Ill. J. Math. 9 (1965), 381-398.

[Go] R. Godement, Théorie des faisceaux, Hermann, Paris, 1957.

[HKRS1] P.M. Hajac, M. Khalkhali, B. Rangipour and Y. Sommerhäuser, Stable anti-Yetter-Drinfeld modules, C. R. Math. Acad. Sci. Paris 338 (2004), 587-590.

[HKRS2] P.M. Hajac, M. Khalkhali, B. Rangipour and Y. Sommerhäuser, Hopf-cyclic homology and cohomology with coefficients, C. R. Math. Acad. Sci. Paris 338 (2004), 667-672.

$[\mathrm{H}] \quad$ D. Hobst, Antipodes in the theory of noncommutative torsors, PhD thesis Ludwig-Maximilians Universität München (2004), Logos Verlag Berlin, 2004.

[JŞ] P. Jara and D. Ştefan, Hopf-cyclic homology and relative cyclic homology of Hopf-Galois extensions, Proc. London Math. Soc. (3) 93 (2006), 138-174.

[Kad] L. Kadison, Cyclic homology of triangular matrix algebras, In: Topology Hawaii (Honolulu, HI, 1990), World Sci. 1992.

[KSz] L. Kadison and K. Szlachányi, Bialgebroid actions on depth two extensions and duality, Adv. Math. 179 (2003), 75-121.

[KLV] S. Kasangian, S. Lack and E. Vitale, Coalgebras, braidings, and distributive laws, Theory and Applications of Categories 13 (2004), 129-146.

[Kass] C. Kassel, Quantum groups. Graduate Text in Mathematics 155, Springer, 1995.

[Kay05] A. Kaygun, Bialgebra cyclic homology with coefficients, K-Theory 34 (2005), 151-194.

[Kay06] A. Kaygun, The universal Hopf cyclic theory, preprint arXiv:math/0609311.

[KR03] M. Khalkhali and B. Rangipour, Invariant cyclic homology, K-Theory 28 (2003), 183-205.

[KR04] M. Khalkhali and B. Rangipour, Para-Hopf algebroids and their cyclic cohomology, Lett. Math. Phys. 70 (2004), 259-272.

[KR05] M. Khalkhali and B. Rangipour, A note on cyclic duality and Hopf algebras, Comm. Algebra 33 (2005), 763-773.

[LPvO] J. Lopez, F. Panaite and F. van Oystaeyen, General twisting of algebras, to appear in Adv. Mathematics.

[Lo] J.-L. Loday, Cyclic Homology. Springer-Verlag 1992.

$[\mathrm{Lu}] \quad$ J.H. Lu, Hopf algebroids and quantum groupoids, Int. J. Math. 7 (1996), 47-70.

[MacL] S. Mac Lane, Categories for the working mathematician. Springer-Verlag 1974.

[Mj] S. Majid, Foundations of quantum group theory. Cambridge University Press 1995.

[Ra] B. Rangipour, Cyclic cohomology of corings, preprint arXiv:math/0607248.

[Sch98] P. Schauenburg, Bialgebras over noncommutative rings and a structure theorem for Hopf bimodules, Appl. Categorical Str. 6 (1998), 193-222.

[Sch00] P. Schauenburg, Duals and doubles of quantum groupoids ( $\times_{R}$-Hopf algebras), in: N. Andruskiewitsch, W.R. Ferrer-Santos and H.-J. Schneider (eds.) AMS Contemp. Math. 267, AMS Providence 2000 pp. 273-293.

[Šk] Z. Škoda, Cyclic structures for simplicial objects from comonads, preprint arXiv:math/0412001.

[Ta] M. Takeuchi, Groups of algebras over $A \otimes \bar{A}$, J. Math. Soc. Japan 90 (1977), 459-492.

[V] P. Vecsernyés, Larson-Sweedler theorem and the role of grouplike elements in weak Hopf algebras, J. Algebra 270 (2003), 471-520. 
[We] C. Weibel, An introduction to homological algebra. Cambridge Studies in Advanced Mathematics 38, Cambridge, University Press, 1994.

Research Institute for Particle and Nuclear Physics, Budapest, H-1525 Budapest 114, P.O.B.49, HUNGARY.

University of Bucharest, Faculty of Mathematics and Informatics, Bucharest, 14 Academiei Street, Ro-010014, Romania. 